\title{
WPS3628
}

\section{Deposit Insurance around the World: A Comprehensive Database}

\author{
Asli Demirgüç-Kunt \\ (World Bank) \\ Baybars Karacaovali \\ (University of Maryland) \\ Luc Laeven* \\ (World Bank and CEPR)
}

\begin{abstract}
World Bank Policy Research Working Paper 3628, June 2005
The Policy Research Working Paper Series disseminates the findings of work in progress to encourage the exchange of ideas about development issues. An objective of the series is to get the findings out quickly, even if the presentations are less than fully polished. The papers carry the names of the authors and should be cited accordingly. The findings, interpretations, and conclusions expressed in this paper are entirely those of the authors. They do not necessarily represent the view of the World Bank, its Executive Directors, or the countries they represent. Policy Research Working Papers are available online at http://econ.worldbank.org.
\end{abstract}

* Demirgüç-Kunt: World Bank (E-mail: ADemirguckunt@WorldBank.org); Karacaovali: University of Maryland (E-mail: Karacaov@econ.umd.edu); Laeven: World Bank, and Centre for Economic Policy Research (E-mail: LLaeven@worldbank.org). We are very grateful to Guillermo Noguera for providing excellent research assistance and to numerous colleagues at the World Bank, the International Association of Deposit Insurers, and officials of deposit insurance agencies, Ministries of Finance, and Central Banks around the world for providing input for the deposit insurance database. 


\begin{abstract}
This paper updates the Demirgüç-Kunt and Sobaci (2001) cross-country deposit insurance database and extends it in several important dimensions. This new dataset identifies both recent adopters and the ones that were not covered earlier due to a lack of data. Moreover, for the first time, it provides historical time series for several variables and adds new ones. The data were collected by surveying deposit insurance institutions and related agencies as well as through the use of various other country sources.
\end{abstract}

Keywords: Deposit insurance; Deposit protection; Deposit coverage; Banking. JEL Classification: G21, G28. 


\section{Introduction}

This paper presents and discusses a new deposit insurance database that updates an earlier one constructed in 1999 by Demirgüç-Kunt and Sobaci (2001) and extends it in several important dimensions.

This new comprehensive database includes 14 new countries that have adopted deposit insurance schemes since $1999^{1}$ and identifies 12 other countries ${ }^{2}$ that had adopted deposit insurance as of 1999 but do not appear in Demirgüç-Kunt and Sobaci (2001) due to lack of data. Apart from the use of various country sources, we have carried out surveys directed to officials of deposit insurance institutions, central banks, and related government officials around the world. The other important contribution of this dataset is the addition of historical time series (rather than data for year-end 1999 only) for several key variables, including deposit insurance coverage, coverage ratios, and co-insurance. The variables are also expanded to include the level of co-insurance requirements, percentage of the value of deposit covered, and whether the payments are per depositor or per depositor per account. Finally, the dataset incorporates part of the survey data relevant for deposit insurance provided by Barth, Caprio and Levine (2004).

Deposit insurance has become an increasingly used tool by governments in an effort to ensure the stability of banking systems and protect bank depositors from incurring large losses due to bank failures. Almost all countries actually have financial safety nets in place which include explicit and implicit deposit insurance, bank regulation and supervision, central bank lender of last resort facilities, and bank insolvency resolution procedures. Although deposit insurance is gaining in popularity among policymakers, its desirability is debated by many economists who point to the moral hazard problems involved and the accompanying excessive risk taking by banks (see, for example, Demirgüç-Kunt and Kane 2002).

This paper aims to support the recently growing empirical literature that deals with the effects of deposit insurance design on different banking outcomes (for example,

\footnotetext{
${ }^{1}$ The new adopters are Albania (2002), Bolivia (2001), Cyprus (2000), Jordan (2000), Malta (2003), Nicaragua (2001), Paraguay (2003), Russia (2003), Serbia and Montenegro (2001), Slovenia (2001), Turkmenistan (2000), Vietnam (2000), Uruguay (2002), and Zimbabwe (2002) where the adoption years are indicated in parentheses.

2 These countries are Algeria, Bahamas, Belarus, Bosnia and Herzegovina, Guatemala, Honduras, Indonesia, Isle of Man, Kazakhstan, Liechtenstein, Malaysia, and Thailand.
} 
Demirgüç-Kunt and Huizinga 2004, Demirgüç-Kunt and Detragiache 2002, and Laeven 2004) by providing detailed data on features of deposit insurance schemes around the world in an empirically usable format. We present the salient features of the data in detail with countries grouped according to income level and geographical region.

The paper is organized as follows. Section 2 discusses the adoption of deposit insurance around the world and section 3 describes the main database. Section 4 discusses main features of the deposit insurance schemes and section 5 concludes. The database, country details and sources are presented in the appendix.

\section{Deposit insurance adoption}

As Demirgüç-Kunt, Kane and Laeven (2005) point out, every country has a de facto implicit deposit insurance scheme (IDIS) in place since governments get pressed for relief at the breakout of a large systemic banking distress. We assume that if an explicit deposit insurance scheme (EDIS) does not exist, then the country has implicit deposit insurance.

Figure 1 displays a map of the world depicting a detailed characterization of deposit insurance adoption around the world as of 2003. The countries with EDIS are colored grey, whereas the countries with IDIS are colored white. Moreover, the figure denotes the countries that provided full guarantees with striped shading and the adopters after 1995 are marked with a star. Figure 2 provides the number of countries with EDIS and IDIS in our sample of 181 countries based on their income level, and Table 1 enlists their names. ${ }^{3}$ Figure 3 and Table 2 provide similar information for middle and lowincome countries where the countries are grouped according to their geographical region. As of 2003, 88 countries adopted EDIS, whereas the remaining 93 countries in our sample are considered to have IDIS (Table 1 and Figure 2). ${ }^{4}$

As shown in Table 3, the adoption of EDIS seems to increase with income level; $16.39 \%$ of low-income countries have an EDIS, whereas the ratio goes up to $60.71 \%$ for upper-middle-income and to $75 \%$ for high-income countries. When the proportion of countries with EDIS is computed based on their GDP, hence how large their economies

\footnotetext{
${ }^{3}$ Gibraltar is excluded from Table 1 and Figure 2 due to lack of data as well as the other tables and figures where countries are grouped by income level.
} 
are, the proportions rise to $96.35 \%$ for high-income countries and to $78.11 \%$ for lowincome countries (Table 3). The proportions based on GDP per capita are very similar to the ones based on the number of countries (Table 3 ).

Among the middle and low-income countries, the occurrence of EDIS seems to be higher in Europe and Central Asia (74.07\%) and Latin America and Caribbean (66.67\%), whereas it is the lowest in Sub-Saharan Africa (10.87\%) (Table 3). The occurrence rates go up to approximately $98 \%$ for both European and Central Asian, and Latin American and Caribbean countries when proportions are based on GDP.

The United States is the first in history to adopt an EDIS which dates back to 1934 - a year marked by a banking crisis. ${ }^{5}$ As shown in Figure 4, this was followed in 1960s by nine other countries and the trend has been dramatically upward especially since 1980s reaching a total of 88 countries in 2003 which is a quadruple of the 1984 figure. In 1994, deposit insurance became the standard for the newly created single banking market of the European Union (EU). Until 1990s the EDISs mostly prevailed and kept building in high income countries but since 1995 we have observed a surge to EDISs in especially lower middle income countries (Figure 4). This is partly driven by the Eastern and Central European transition economies which eventually became or are expected to become EU members although EDISs remain quite prevalent in Latin America and Caribbean as well, thanks to the generally accepted best practice advice given to the developing countries (Folkerts-Landau and Lindgren 1998, and Garcia 1999).

\section{The database}

The database builds on Demirgüç-Kunt and Sobaci (2001) as mentioned in the introduction. A large section of their database was constructed by the survey results of an International Monetary Fund working paper (Garcia 1999) and earlier sources such as Kyei (1995) and Talley and Mas (1990) augmented by some other country sources. We further complement and improve the database through various other country and online

\footnotetext{
${ }^{4}$ There is no data available for Andorra, Monaco, San Marino, and Vatican City so they are not included in the dataset.

${ }^{5}$ In Norway there was a guarantee fund for savings banks with voluntary membership in 1921 which became obligatory in 1924, whereas a guarantee fund for commercial banks was first introduced in 1938 (Gerdrup 2003). However, Norway's guarantee fund is not considered a pure deposit insurance scheme so they had no official explicit deposit insurance until 1961.
} 
sources as well as a survey of deposit insurers. One of the main improvements is the introduction of historical data on coverage and co-insurance, introducing a time series aspect to the data. Another major data source is the survey carried out by the International Association of Deposit Insurers in 2002-03. The main cross-country part of the database comprises readily usable data for empirical and statistical analysis where most variables are coded as indicators along with explanatory details. We present the main database in the appendix section A.1. The details of the data for each country with references to the sources are covered in the appendix section A.2 and the detailed data sources are given in the appendix section A.3.

The electronic version of the full dataset $^{6}$ is available online at the Finance Research website of the Development Economics Research Group, World Bank. The complete database includes the full coverage ratio data spanning 1960 to 2003 for all countries, where applicable. In the following sections we describe the dataset and the included variables and discuss main features of explicit deposit insurance systems around the world.

\subsection{Explicit versus implicit deposit insurance}

EDISs differ from IDISs due to their reliance on formal regulation through central bank law, banking law, or the constitution and so on. The relevant law explains the main ingredients of the deposit insurance such as the beginning date, coverage limits, how (if any) they are going to be funded, and how bank failures will be resolved.

If such regulation is not present for deposit insurance, we assume that the DIS is implicit, relying on the observation that every country establishes a de facto insurance system for banks.

The variables related to the type of deposit insurance available in each country comprise of the following: a) Type: This variable identifies the form of the deposit insurance - explicit or implicit - present in each country. The variable takes the value of one for countries with EDIS, and zero otherwise (Table A.1.1). b) Date Enacted /

\footnotetext{
${ }^{6}$ The data is available as an Excel workbook consisting of three worksheets. The first worksheet includes the main cross-country dataset, the second worksheet provides historical levels of coverage limits and coinsurance, and finally the third worksheet provides the coverage ratios (coverage limits as a share of GDP per capita).
} 
Revised: This variable provides the year in which an EDIS was first enacted along with the year in which the system was later revised, if applicable (Table A.1.2).

\subsection{Coverage}

EDISs vary in their extent and amount of coverage. EDISs also differ in the types of deposits and institutions they apply to. For example, countries which would like to protect their payments systems only, limit the guarantee of EDISs to deposits with commercial banks and to other depository institutions providing payment transactions. On the other hand some EDISs may extend guarantees to other types of institutions such as savings banks, if they involve a wide-ranging objective.

Some countries have adopted different sets of EDISs that apply to different types of institutions. Usually there exists one EDIS for commercial banks and one for other deposit taking institutions. For example, Japan, France, Germany, and Norway have two separate EDISs, whereas Spain has three. For countries that have more than one EDIS, the database provides information on the EDIS for commercial banks only. However, in section A.2., we provide detailed information on each country's system along with relevant laws and names of institutions.

Depending on the objective of the EDIS, the coverage varies based on different types of deposits. In most cases, foreign deposits of domestic banks, domestic deposits of foreign banks, inter-bank deposits, and deposits denominated in foreign currencies are not covered under the EDISs. The database provides information on the coverage for inter-bank deposits, and foreign currency denominated deposits.

\subsubsection{Foreign currency deposit coverage}

The variable named "Foreign Currencies" takes the value one for systems that cover foreign currency denominated deposits, and zero otherwise (Table A.1.2). However, some EDISs are restrictive in the set of foreign currencies they cover. For instance, Hungary extends coverage to deposits denominated in EUR or currencies of other OECD countries. ${ }^{7}$ This variable takes the value one for such countries as well.

\footnotetext{
${ }^{7}$ The details for each country are discussed in section A.2.
} 


\subsubsection{Inter-bank deposit coverage}

The EDISs mostly do not cover inter-bank deposits since unlike small depositors, banks are perceived to have enough resources to monitor other banks. Thus, extending coverage to inter-bank deposits could reduce the incentives to supervise other banks and undermine the market discipline. The countries with inter-bank deposit coverage are listed in Table 4 grouped by income level. The only two high-income countries with this feature are Canada and United States. Interestingly, some eight lower-middle-income countries also provide it (Table 4).

In the database, the variable named "Inter-Bank Deposits" takes the value one for EDISs that extend coverage to inter-bank deposits and zero otherwise (Table A.1.2).

\subsubsection{Amount of coverage}

The amount of coverage matters since it directly affects the market discipline exerted by depositors. If the coverage is low, then better and more reliable banks will be preferred by depositors. On the other hand, this is partly against the objectives of the deposit insurance that protects small depositors who lack the resources to evaluate the soundness of banks. However, very high coverage limits could inhibit any form of monitoring on the depositors' end and downplay market discipline.

In Table A.1.2, the following variables on the amount of coverage are listed: a) Coverage Limit as of 2003: This variable provides the details on the amount of coverage and co-insurance. More specifically, the provided information includes the currency in which the coverage is reported, the coverage limit and whether it is a full coverage; the percentage of the deposits covered if co-insurance exists and the structure of co-insurance. b) Coverage Limit as of 2003 in US\$: Expresses the coverage limit in US dollars.

Some countries provide unlimited coverage which usually emerges in response to banking crises. For example, as of 2003 Dominican Republic, Indonesia, Malaysia, Thailand, Turkey, and Turkmenistan had full guarantees. Similarly, other countries, such as Ecuador, Japan, and Mexico, had full coverage in the past that was revoked after the crises seemed to abate. The historical series of the coverage provided are presented in Table A.1.7 and are discussed further below in section 3.5. 


\subsubsection{Co-insurance}

Some countries have adopted co-insurance mechanisms that require depositors to bear part of the cost in case of a banking failure. Thus, it is aimed to get depositors to make more prudent bank choices in their deposit decision. As of 2003 there were 21 EDISs with co-insurance. Table 5 lists these countries and the co-insurance requirement by depositors for each country. Co-insurance does not exist in low-income countries but otherwise gets more and more prevalent the higher the income level (Table 5).

In Table A.1.3, the following variables related to co-insurance are listed: a) Coinsurance: This variable takes the value of one if the country requires a co-insurance, and zero otherwise. b) Co-insurance percentage: This variable provides the percentage of the deposit amount the depositors are responsible for and hence lose in case of a bank failure.

The historical values of the co-insurance requirements are given in Table A.1.7 and are discussed further below in section 3.5.

\subsubsection{Extent of coverage}

The EDISs differ in terms of the extent of their coverage as well. In most countries the coverage is per depositor which means that the sum of deposits per depositor is protected up to the applicable limit. However, some countries provide protection per depositor per account, hence the actual amount of coverage is higher for persons with multiple accounts. In Table A.1.3 the variable "Payment" takes the value one if the protection is per depositor and zero if it is per depositor per account.

\subsubsection{Coverage distribution}

We observe varying degrees of deposit values being covered in different EDISs across the world. In Table A.1.3 the variable "Percentage of deposit value covered" provides the extent of total protection coverage as a share of total deposit value in each country. This variable takes the maximum value of $100 \%$ for countries that provide full coverage and is less than $100 \%$ for the rest, which average around $48 \%$. In Table A.1.3 the source of information and the reference year on this coverage distribution is also provided under the variables labeled "Information source of coverage distribution" and "Reference date of data on coverage distribution", respectively. 


\subsection{Funding}

EDISs can be either funded or unfunded. In funded systems the member institutions need to make periodic contributions to the fund, which is then used as the main source for paying out depositors during bank failures. In a minority of the countries, which mainly belong to the high-income category, there are unfunded systems, where members have to contribute to the fund after the failure. Chile is an exception, where the government is the sole contributor of the fund. As of 2003, only 14 countries $^{8}$ out of 88 had unfunded EDISs and 11 of these countries was European.

\subsubsection{Premiums}

In Table A.1.4, the variable labeled "Annual Premiums" provides information on the premiums required as a percentage of the base as well as whether it involves a variable or fixed rate and is risk-based.

Assessment bases for premiums vary across different systems. Premiums are generally based on deposits and insured deposits. However, some systems are based on domestic or all obligations of the banks. The related variable is listed in Table A.1.4 and is named "Premium or assessment base".

Premiums may vary according to the riskiness of the assessment base which are then called risk-adjusted premiums. As of 1995 only United States had a system with risk-adjusted premiums. Since then, the number of countries with risk-adjusted DISs has gone up to 20, which are listed according to income category in Table 6. In Table A.1.4 the variable labeled "Risk-adjusted premiums" takes the value one if premiums are riskadjusted, and zero otherwise.

\subsubsection{Funding source, administration and membership}

Public funding may be available in addition to premiums contributed by banks. Public funds may be initial contributions or losses taken ex-post by the government or they might simply be in the form of central bank loans. The funds might also be a combination of both private and public. In Table A.1.5 the variable labeled "Source of funding" takes the value of two if the EDIS is funded by the government only, zero if funded privately only, and zero if jointly funded.

\footnotetext{
${ }^{8}$ Countries with unfunded EDISs are: Austria, Bahrain, Chile, France, Gibraltar, Isle of Man, Italy, Liechtenstein, Luxembourg, Netherlands, Slovenia, Switzerland, Thailand, and United Kingdom.
} 
The variable "Administration" in Table A.1.5 takes on three values; one if the administration of the fund is official, two if it is joint, and three if it is private. If the EDIS of a country is administered by the central bank, it is considered to have an official administration. Moreover, some privately administered institutions have limited authorities. For example, in Italy and Croatia certain decisions need to go through the central bank approval, hence the EDISs of these countries are considered to have a joint administration in the database.

Finally, the variable "Membership" in Table A.1.5 takes the value one if the membership to the fund is compulsory and zero if it is voluntary. Majority of the countries have compulsory membership, whereas only ten percent of them employ a voluntary system. ${ }^{9}$

\subsection{Barth, Caprio, and Levine (2004) survey questions}

We also incorporate the deposit insurance related survey results from Barth, Caprio, and Levine (2004) database on banking regulation and supervision. All of the data is coded for empirical use and presented in three different panels in Table A.1.6. ${ }^{10}$

The variables in this section and the way they are coded are as follows: 1) Does the deposit insurance authority make the decision to intervene a bank? The answer "Yes" is coded with one and "No" with zero (panel A). 2) Does the deposit insurance authority have the legal power to cancel or revoke deposit insurance for any participating bank? The answer "Yes" is coded with one and "No" with zero (panel A). 3) As part of failure resolution, how many banks closed or merged in the last 5 years? The number of banks is reported (panel A). 4) Were depositors wholly compensated (to the extent of legal protection) the last time a bank failed? The answer "Yes" is coded with one and "No" with zero (panel A). 5) On average, how long does it take to pay depositors in full? The number of months is reported (panel B). 6) What was the longest that depositors had to wait in the last 5 years? The number of months is reported (panel B). 7) Were any deposits not explicitly covered by deposit insurance at the time of the failure compensated when the bank failed (excluding funds later paid out in liquidation

\footnotetext{
${ }^{9}$ The membership is voluntary in the following countries: Dominican Republic, Kazakhstan, Marshall Islands, Micronesia, Sri Lanka, Switzerland, and Taiwan.

${ }^{10}$ The countries which did not provide answers for any of the survey questions are excluded from the Table. Please see Table A.1.6 (any panel) for a complete list of participants.
} 
procedures)? The answer "Yes" is coded with one and "No" with zero (panel B). 8) Can the deposit insurance agency/fund take legal action against bank directors or other bank officials? The answer "Yes" is coded with one and "No" with zero (panel C). 9) Has the deposit insurance agency/fund ever taken legal action against bank directors or other bank officials? The answer "Yes" is coded with one and "No" with zero (panel C). 10) Are non-residents treated differently than residents with respect to deposit insurance scheme coverage? The answer "Yes" is coded with one and "No" with zero (panel C).

\subsection{Time series: Coverage limits, co-insurance, and coverage ratios}

The database includes time series data for co-insurance and coverage limits. The limits and the co-insurance requirements since the year of EDIS adoption and the revisions to them over time are presented in Table A.1.7. The amount of coverage is seen to vary across different schemes. They are also adjusted through time to account for inflation as well as changing economic conditions. Table A.1.7 provides the coverage limits, the currency they are measured in and the co-insurance percentages.

Finally, the database provides ratios of coverage amounts to GDP per capita and deposits per capita, where all are expressed in local currency units. The sample years span 1960 to 2003 in the main database online. ${ }^{11}$ The underlying data, that is GDP per capita, total deposits, population, and coverage amounts, are also reported there. In Table A.1.8 we present the two coverage ratios for 1999-2003. Figure 6 provides the ratio of deposit coverage to GDP per capita in 2002 for selected countries. We see that the ratio is quite high for some developing countries. For example, the coverage amount is about ten times larger than the per capita income for the Former Yugoslav Republic of Macedonia. This ratio is even starker for Nicaragua, where it is about twenty-seven in 2002 which appears in Table A.1.8. ${ }^{12}$ The generosity of schemes if not matched with institutional improvement can result in more fragility of financial systems.

\footnotetext{
${ }^{11}$ The third worksheet of the database includes the coverage ratios. The online database is located at the Finance Research website under datasets, World Bank.

${ }^{12}$ Nicaragua is not included in Figure 2 due to space limitations.
} 


\section{Main features of the deposit insurance schemes around the world}

The main features of the schemes are summarized in Table 7, where countries are grouped based on their income level. The middle and low income countries are further subdivided according to their geographical region. This section presents the observations based on Table 7. Panel A provides the number of countries with each listed feature for different income and regional categories. Panel B provides the proportion of countries with each feature in a given category. Panels $\mathrm{C}$ and $\mathrm{D}$ provide the proportion of countries with each feature weighted by their GDP and GDP per capita, respectively.

Foreign currency deposit coverage is prevalent in $76 \%$ of the countries; whereas it is observed in $57 \%$ of the low-income countries and only $25 \%$ of the low and middleincome countries located in Middle East and North Africa. The ratios weighted by GDP and GDP per capita are also similar with the exception of Middle East and North Africa region, where the ratio goes up to 50\% with GDP per capita. Extension of coverage to inter-bank deposits is not very common, amounting to 13 out of 80 countries (16\%) with data for this variable. It is mostly observed in lower middle and low-income countries (29\% in each), and among them mostly in the Asia and Pacific region (57\% of them). Coinsurance is not required by low-income countries and is otherwise required by about a third of the countries. Among the middle-income countries, it is most prevalent in the Middle East and North Africa region. Most countries, $79 \%$ in total, calculate the coverage on a per depositor (per institution) basis.

Almost all schemes are permanently funded except the ones in high-income category, where $37 \%$ of them have no permanent fund and contributions are usually called upon, if deemed necessary, on an ex-post basis. Premiums are not risk-adjusted in the low-income category and it is also uncommon in other categories where some $23 \%$ of the countries employ this feature. Membership to the schemes is compulsory in $90 \%$ of the countries. The only exception is the Asia and Pacific Region, where 50\% of the group has a voluntary membership. The funding is pre-dominantly provided jointly by private and public resources, in $63 \%$ of the countries. Only Chile has a sole public funding but in most countries, government at least provides the initial capital if not the subsequent funding needs. Sole private funding is more widespread in the high income category, where half of them have a privately funded system. The schemes are mostly administered officially (60\%), followed by joint administration (26\%). Private administration is highest 
in the high income category, where $23 \%$ of the group has a privately administered system.

\section{Conclusion}

This comprehensive database provides detailed information on the deposit insurance schemes across the world as of 2003. It improves significantly over the earlier DemirgüçKunt and Sobaci (2001) cross-country database. First, the database includes 14 new countries that have adopted deposit insurance schemes since 1999 and identifies 12 other countries with DISs as of 1999 that were not covered before. Second, the database uses various country sources and surveys of deposit insurance agencies and officials around the world, and hence completes and further details the other collected data. Third, this dataset adds historical time series data, and covers the values of deposit insurance coverage amounts, co-insurance, and coverage ratios since the inception of the first nationwide scheme by the United States in 1934. Fourth, other new variables are incorporated that include the level of co-insurance requirements, percentage of the value of deposits covered, and whether the payments are per depositor or per depositor per account.

The work here is part of a broader research project in understanding and characterizing the design, and implementation of deposit insurance as analyzed in Demirgüç-Kunt, Kane and Laeven (2005) using this data. Moreover, it will help and hopefully stimulate further research on the effect of deposit insurance on financial development, financial stability, fragility and market discipline. We provide the data in an empirically usable format to contribute to this growing literature. 


\section{References}

Barth, James R., Gerard Caprio, and Ross Levine. 2004. "The Regulation and Supervision of Banks around the World: A New Database," 2003 Version and Update, Washington, DC: World Bank.

Demirgüç-Kunt, Asli, and Enrica Detragiache. 2002. "Does Deposit Insurance Increase

Banking System Stability? An Empirical Investigation," Journal of Monetary Economics, 49(7): 1373-1406.

Demirgüç-Kunt, Asli, and Harry Huizinga. 2004. "Market Discipline and Deposit Insurance," Journal of Monetary Economics, 51(2): 375-399.

Demirgüç-Kunt, Asli, and Edward Kane. 2002. "Deposit Insurance Around the World:

Where Does it Work?” Journal of Economic Perspectives 16, 175-195.

Demirgüç-Kunt, Asli, Edward Kane, and Luc Laeven. 2005. "Determinants of DepositInsurance Adoption and Design," mimeo, World Bank.

Demirgüç-Kunt, Asli, and Tolga Sobaci. 2001. “A New Development Database: Deposit Insurance around the World," World Bank Economic Review, 15: 481-490.

Folkerts-Landau, David, and Carl-Johan Lindgren. 1998. "Toward a Framework for Financial Stability," mimeo, International Monetary Fund.

Garcia, Gillian. 1999. "Deposit Insurance: Actual and Best Practices," IMF Working Paper 99/54, Washington, DC: International Monetary Fund.

Garcia, Gillian. 2000. "Deposit Insurance: Actual and Good Practices," Occasional Paper No. 197, Washington, DC: International Monetary Fund.

Gerdrup, Karsten R. 2003. "Three Episodes of Financial Fragility in Norway since the 1890s,” BIS Working Paper No. 142, Basel: Bank for International Settlements.

Kyei, Alexander. 1995. "Deposit Protection Arrangements: A Comparative Study," IMF Working Paper 95/134, Washington, DC: International Monetary Fund.

Laeven, Luc. 2004. "The Political Economy of Deposit Insurance,” Journal of Financial Services Research 26(3), 201-224.

Talley, Samuel H., and Ignacio Mas. 1990. "Deposit Insurance in Developing Countries," Policy Research Working Paper, No. 548, Washington, DC: World Bank. 
Figure 1. Adoption of deposit insurance around the world (as of 2003)

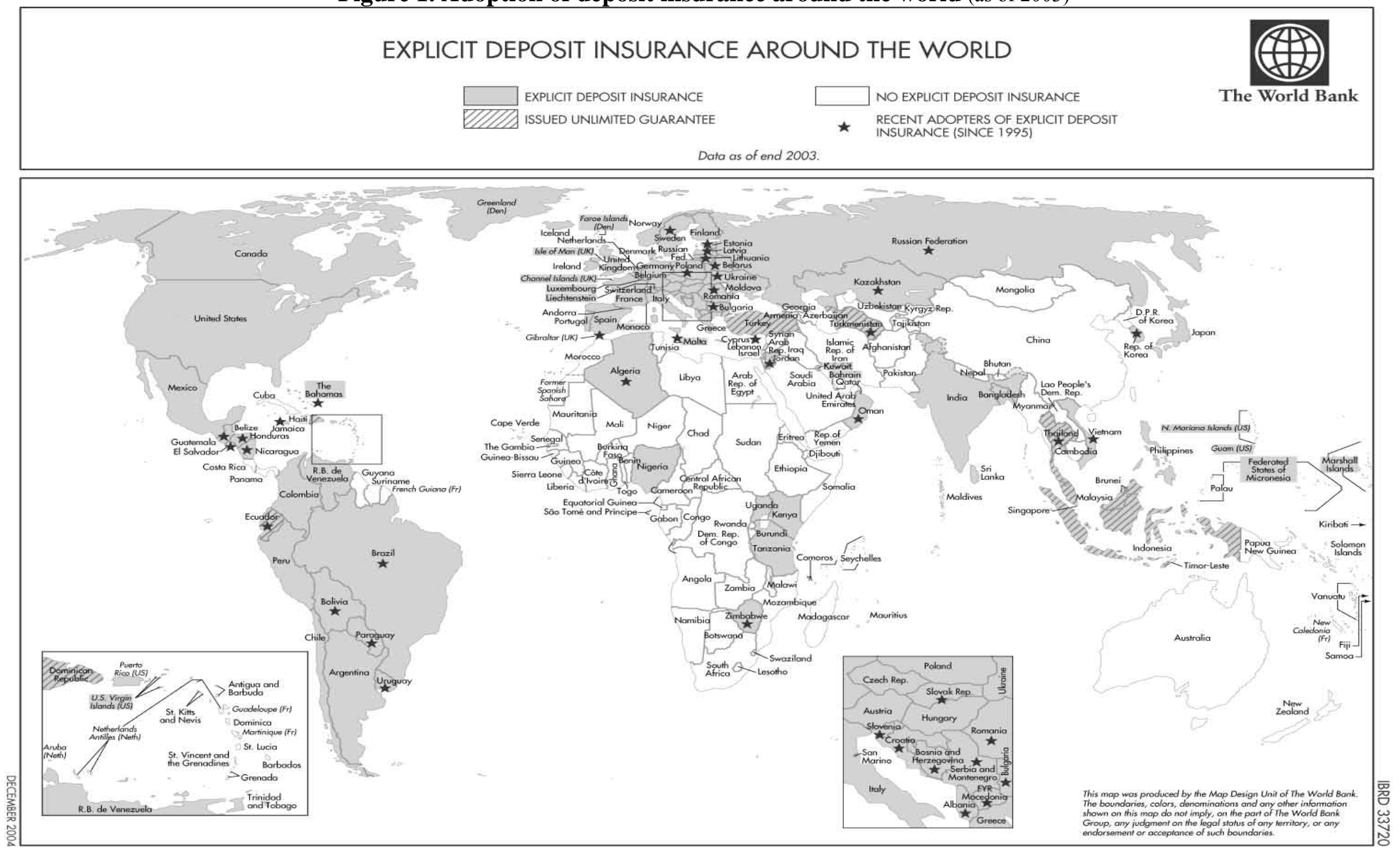


Figure 2. Adoption of deposit insurance around the world by income level (as of 2003)

Panel A: Explicit deposit insurance

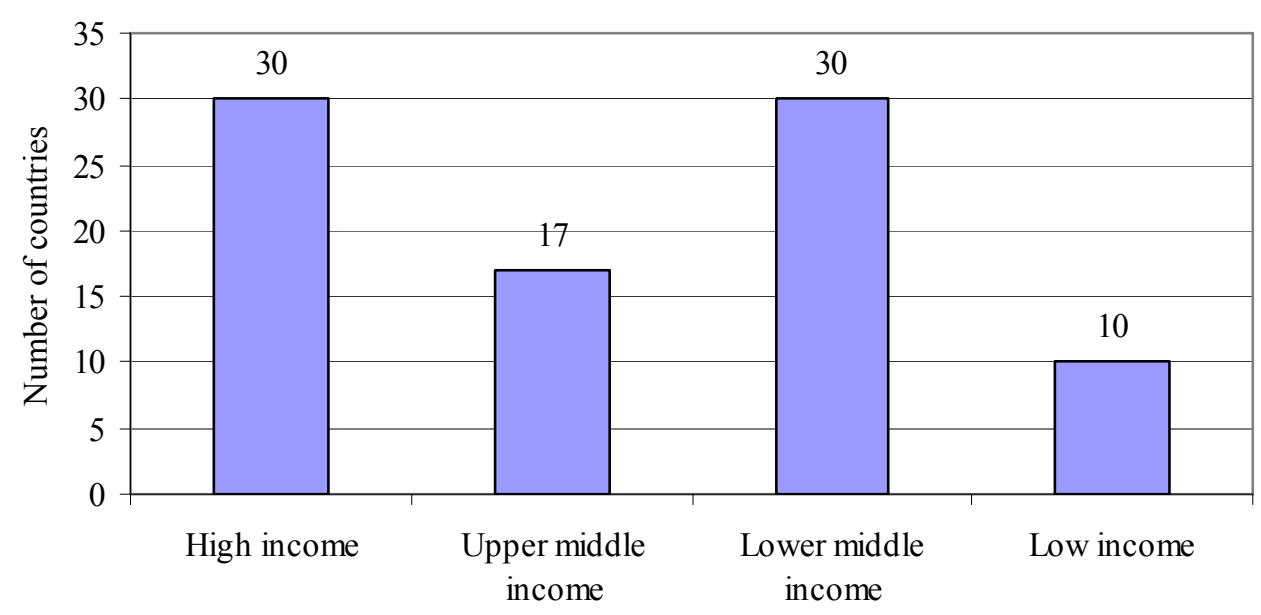

Panel B: Implicit deposit insurance

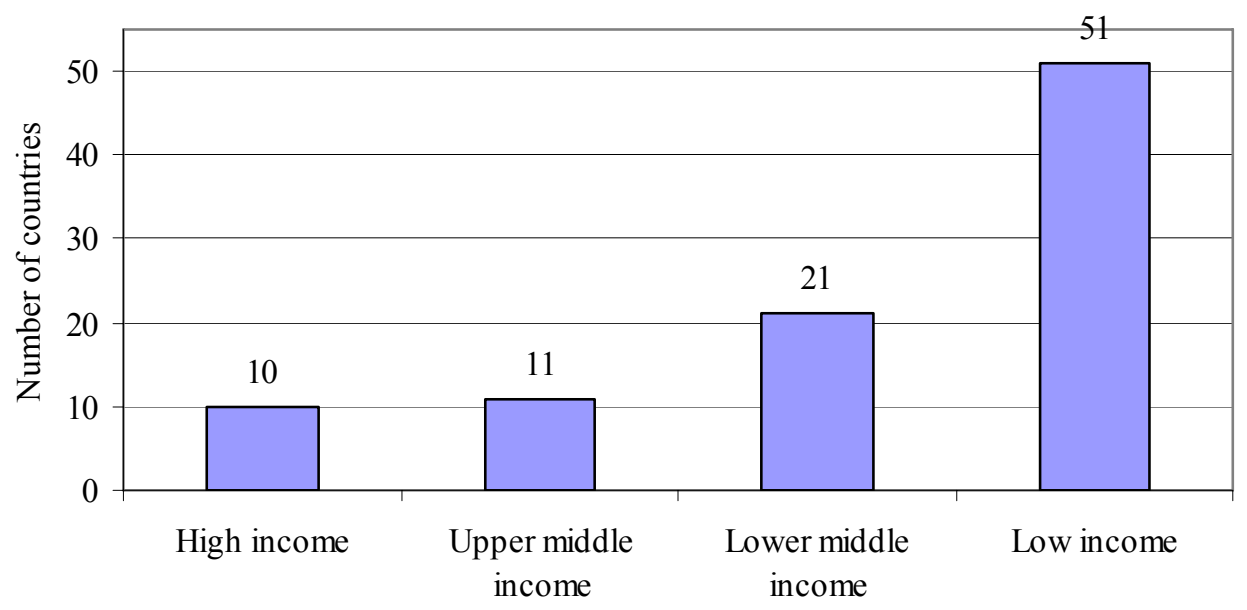


Figure 3. Adoption of deposit insurance around the world by region* (as of 2003)

Panel A: Explicit deposit insurance

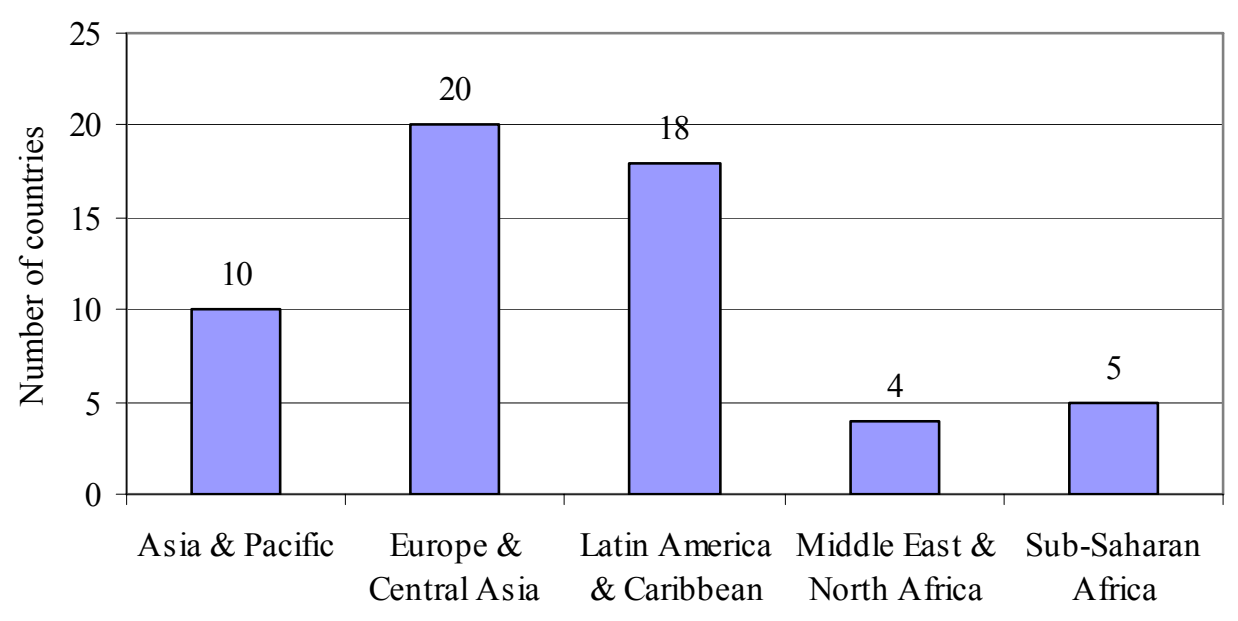

Panel B: Implicit deposit insurance

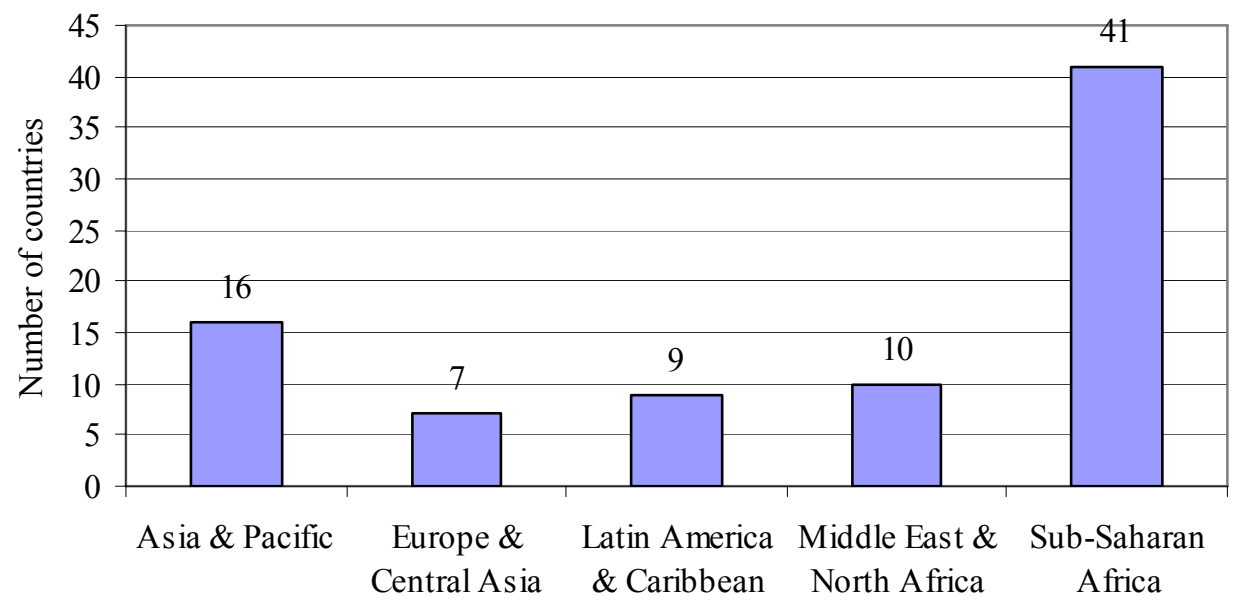

* High income countries are excluded from the analysis 
Figure 4. Trends in the adoption of explicit deposit insurance by income level

Panel A: All income categories

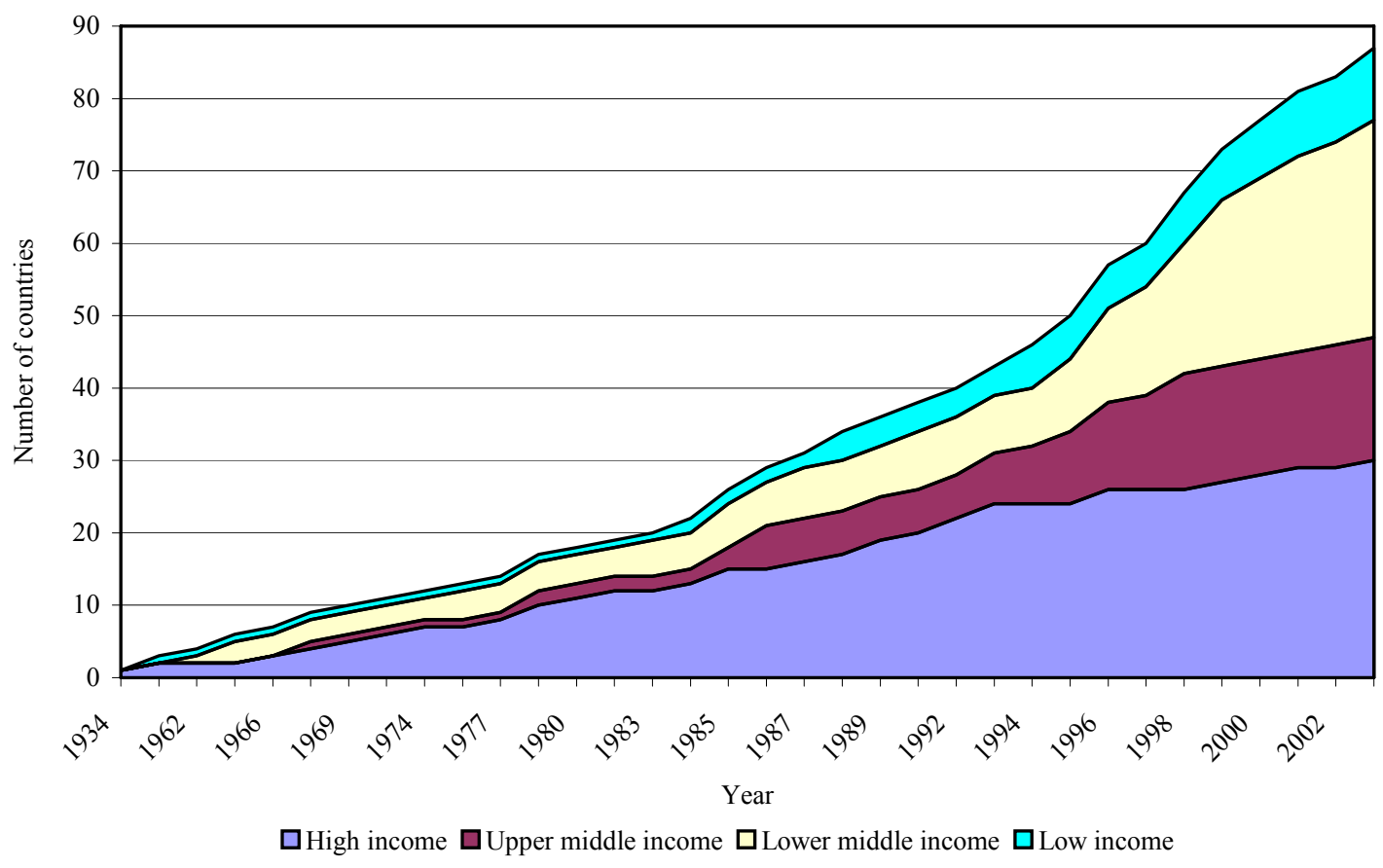

Panel B: High income category versus others

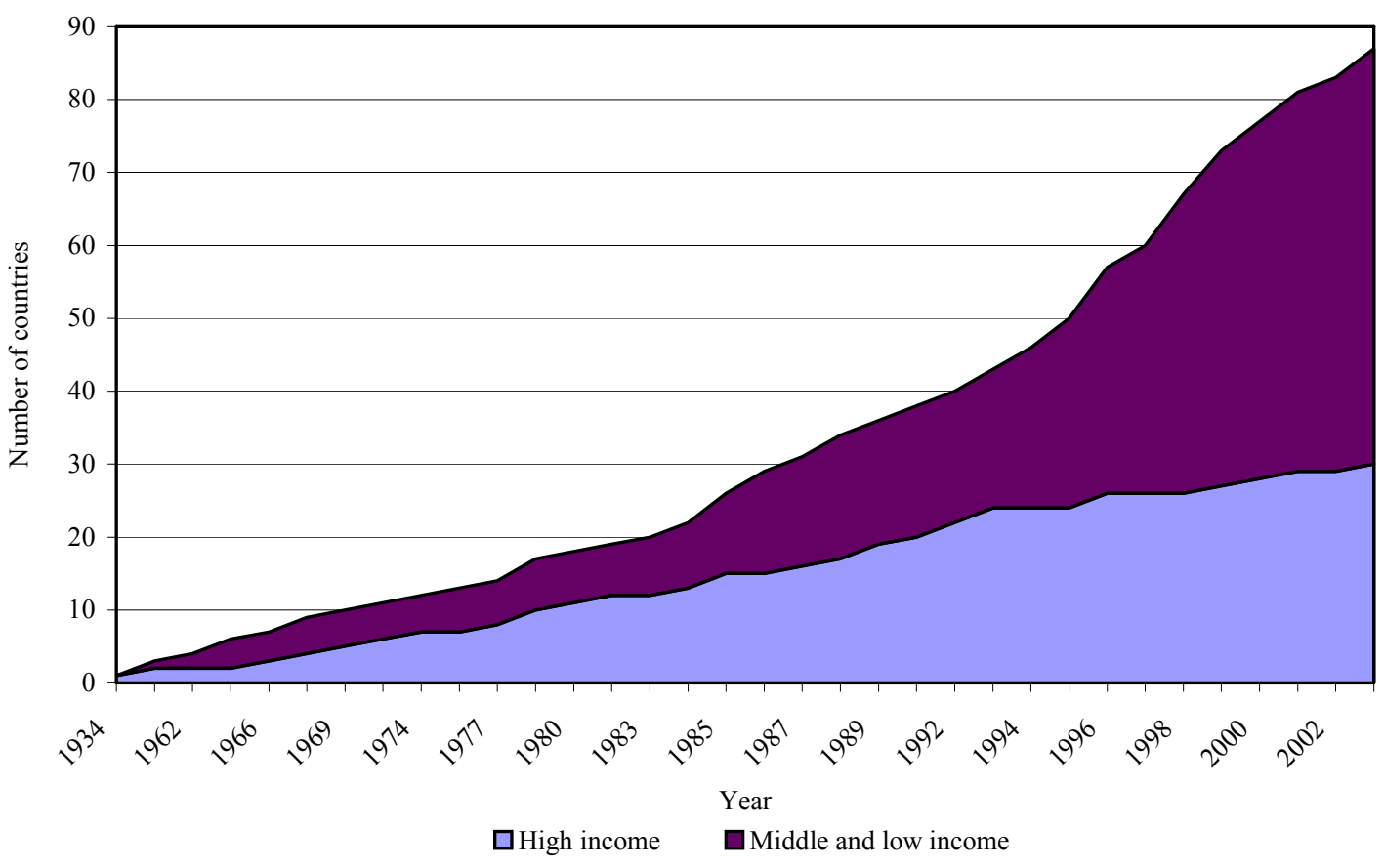


Figure 5. Trends in the adoption of explicit deposit insurance by region*

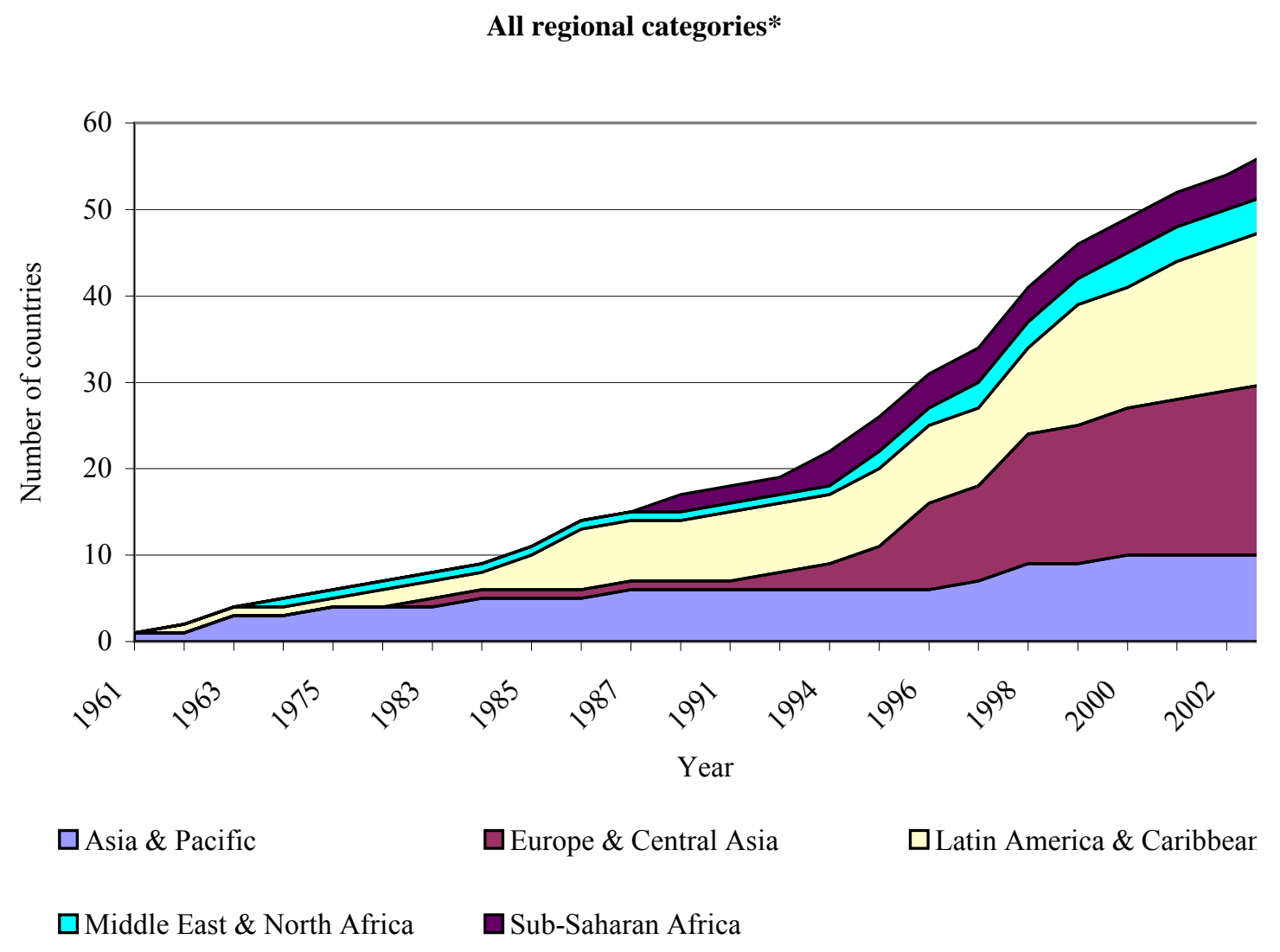

*High income countries are excluded from the analysis 
Figure 6. Ratios of deposit coverage to GDP per capita in selected countries, 2002

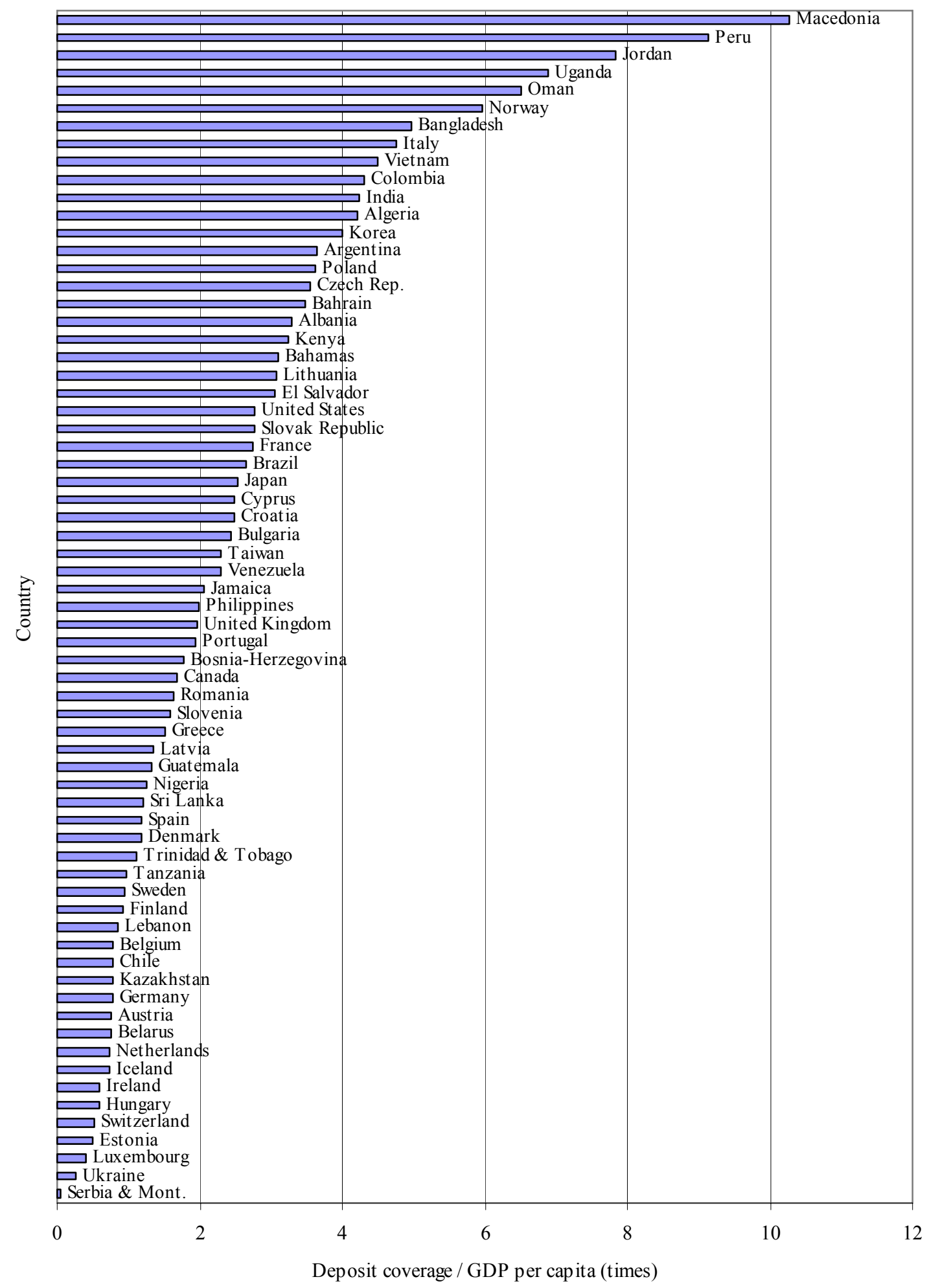


Table 1. Adoption of deposit insurance around the world by income level

(Years of establishment/revision in parentheses and number of countries by group in brackets, as of 2003)

Panel A: Explicit Deposit Insurance

\begin{tabular}{|c|c|c|c|}
\hline \multicolumn{4}{|l|}{ High income countries [30] } \\
\hline Austria $(1979 / 1996)$ & France $(1980 / 1986 / 1999)$ & Korea (1996) & Spain $(1977 / 1996)$ \\
\hline Bahamas (1999) & Germany $(1966 / 1969 / 1998)$ & Liechtenstein $(1992 / 2003)$ & Sweden (1996) \\
\hline Bahrain (1993) & Greece $(1995 / 2000)$ & Luxembourg (1989) & Switzerland (1984/1993) \\
\hline Belgium (1974/1995/1998) & Iceland $(1985 / 1996)$ & Malta (2003) & Taiwan (1985) \\
\hline Canada (1967) & Ireland $(1989 / 1995)$ & Netherlands (1978/1996/1998) & United Kingdom $(1982 / 1995)$ \\
\hline Cyprus (2000) & Isle of Man (1991) & Norway $(1961 / 1997)$ & United States $(1934 / 1991)$ \\
\hline Denmark $(1987 / 1995)$ & Italy $(1987 / 1996)$ & Portugal (1992/1995) & \\
\hline Finland $(1969 / 1992 / 1998)$ & Japan (1971) & Slovenia $(2001)$ & \\
\hline \multicolumn{4}{|c|}{ Upper middle income countries [17] } \\
\hline Argentina $(1979 / 1995)$ & Hungary (1993) & Mexico $(1986 / 1990 / 1999)$ & Uruguay (2002) \\
\hline Chile (1986) & Latvia (1998) & Oman (1995) & Venezuela $(1985 / 2001)$ \\
\hline Croatia (1997) & Lebanon (1967) & Poland (1995) & \\
\hline Czech Rep. (1994) & Lithuania (1996) & Slovak Republic (1996/2001) & \\
\hline Estonia (1998) & Malaysia (1998) & Trinidad \& Tobago (1986) & \\
\hline \multicolumn{4}{|c|}{ Lower middle income countries [30] } \\
\hline Albania (2002) & Colombia (1985) & Kazakhstan $(1999 / 2003)$ & Russia (2003) \\
\hline Algeria (1997) & Dominican Republic (1962) & Macedonia (1996/2000/2002) & Serbia and Montenegro (2001) \\
\hline Belarus & Ecuador (1998) & Marshall Islands (1975) & Sri Lanka (1987) \\
\hline$(1996 / 1998 / 2000 / 2001 / 2004)$ & El Salvador (1999) & Micronesia (1963) & Thailand (1997) \\
\hline Bolivia (2001) & Guatemala (1999) & Paraguay (2003) & Turkey $(1983 / 2000)$ \\
\hline Bosnia-Herzegovina (1998) & Honduras (1999) & Peru (1991) & Turkmenistan (2000) \\
\hline Brazil (1995/2002) & Jamaica (1998) & Philippines (1963) & Ukraine (1998) \\
\hline Bulgaria $(1996 / 1998 / 2001 / 2002)$ & Jordan $(2000)$ & Romania (1996) & \\
\hline \multicolumn{4}{|l|}{ Low income countries [10] } \\
\hline Bangladesh (1984) & Kenya (1988) & Tanzania (1994) & Zimbabwe (2003) \\
\hline India (1961) & Nicaragua (2001) & Uganda (1994) & \\
\hline Indonesia (1998) & Nigeria $(1988 / 1989)$ & Vietnam (2000) & \\
\hline
\end{tabular}

Panel B: Implicit deposit insurance

\begin{tabular}{|c|c|c|c|c|c|}
\hline \multicolumn{6}{|c|}{ High income countries [10] } \\
\hline Australia & Brunei & Israel & New Zealand & Singapore & \\
\hline Barbados & Hong Kong & Kuwait & Qatar & United Arab Emirates & \\
\hline \multicolumn{6}{|c|}{ Upper middle income countries [11] } \\
\hline Belize & Costa Rica & Grenada & Mauritius & Saudi Arabia & St. Lucia \\
\hline Botswana & Gabon & Libya & Panama & Seychelles & \\
\hline \multicolumn{6}{|c|}{ Lower middle income countries [21] } \\
\hline Armenia & Djibouti & Iran & Morocco & Swaziland & W. Samoa \\
\hline Cape Verde & Egypt & Iraq & Namibia & Syria & \\
\hline China & Fiji & Kiribati & South Africa & Tunisia & \\
\hline Cuba & Guyana & Maldives & Suriname & Vanuatu & \\
\hline \multicolumn{6}{|c|}{ Low income countries [51] } \\
\hline Afghanistan & Central African Rep. & Ghana & Malawi & Pakistan & Tajikistan \\
\hline Angola & Chad & Guinea & Mali & Papua New Guinea & Togo \\
\hline Azerbaijan & Comoro Is. & Guinea-Bissau & Mauritania & Rep. of Congo & Uzbekistan \\
\hline Benin & Cote d'Ivoire & Haiti & Moldova & Rwanda & Yemen \\
\hline Bhutan & Equatorial Guinea & \multicolumn{2}{|c|}{ Kyrgyz Republic Mongolia } & Senegal & Zaire \\
\hline Burkina Faso & Eritrea & Laos & Mozambique & Sierra Leone & Zambia \\
\hline Burundi & Ethiopia & Lesotho & Myanmar & Solomon Is. & \\
\hline Cambodia & Gambia & Liberia & Nepal & Somalia & \\
\hline Cameroon & Georgia & Madagascar & Niger & Sudan & \\
\hline
\end{tabular}


Table 2. Adoption of deposit insurance around the world by region as of 2003*

(Years of establishment/revision in parentheses and number of countries by group in brackets, as of 2003)

Panel A: Explicit Deposit Insurance

\begin{tabular}{llll}
\hline Asia \& Pacific [10] & & & \\
\hline Bangladesh (1984) & Malaysia (1998) & Philippines (1963) & Vietnam (2000) \\
India (1961) & Marshall Islands (1975) & Sri Lanka (1987) & \\
Indonesia (1998) & Micronesia (1963) & Thailand (1997) & \\
\hline Europe \& Central Asia [20] & & & Turkey (1983/2000) \\
\hline Albania (2002) & Czech Rep. (1994) & Macedonia (1996/2000/2002) & Turkmenistan (2000) \\
Belarus & Estonia (1998) & Poland (1995) & Ukraine (1998) \\
(1996/1998/2000/2001/2004) & Hungary (1993) & Romania (1996) & \\
Bosnia-Herzegovina (1998) & Kazakhstan (1999/2003) & Russia (2003) & Trinidad \& Tobago (1986) \\
Bulgaria (1996/1998/2001/2002) & Latvia (1998) & Serbia and Montenegro (2001) & \\
Croatia (1997) & Lithuania (1996) & Slovak Republic (1996/2001) & \\
\hline Latin America \& Caribbean [18] & & & Uruguay (2002) \\
Argentina (1979/1995) & Dominican Republic (1962) & Jamaica (1998) & Venezuela (1985/2001) \\
Bolivia (2001) & Ecuador (1998) & Mexico (1986/1990/1999) & \\
Brazil (1995/2002) & El Salvador (1999) & Nicaragua (2001) & \\
Chile (1986) & Guatemala (1999) & Paraguay (2003) & \\
Colombia (1985) & Honduras (1999) & Peru (1991) & \\
\hline Middle East \& North Africa [4] & & & Lebanon (1967) \\
\hline Algeria (1997) & Jordan (2000) & & \\
\hline Sub-Saharan Africa [5] & & Zimbabwe (2003) & \\
\hline Kenya (1988) & Tanzania (1994) & & \\
Nigeria (1988/1989) & Uganda (1994) & & \\
\hline
\end{tabular}

Panel B: Implicit deposit insurance

\begin{tabular}{|c|c|c|c|c|c|}
\hline \multicolumn{6}{|c|}{ Asia \& Pacific [16] } \\
\hline Afghanistan & China & Laos & Myanmar & Papua New Guinea & W. Samoa \\
\hline Bhutan & Fiji & Maldives & Nepal & Solomon Is. & \\
\hline Cambodia & Kiribati & Mongolia & Pakistan & Vanuatu & \\
\hline \multicolumn{6}{|c|}{ Europe \& Central Asia [7] } \\
\hline Armenia & Georgia & Moldova & Uzbekistan & & \\
\hline Azerbaijan & Kyrgyz Republic & Tajikistan & & & \\
\hline \multicolumn{6}{|c|}{ Latin America \& Caribbean [9] } \\
\hline Belize & Cuba & Guyana & Panama & Suriname & \\
\hline$\underline{\text { Costa Rica }}$ & Grenada & Haiti & St. Lucia & & \\
\hline \multicolumn{6}{|c|}{ Middle East \& North Africa [10] } \\
\hline Djibouti & Iran & Libya & Saudi Arabia & Tunisia & \\
\hline Egypt & Iraq & Morocco & Syria & Yemen & \\
\hline \multicolumn{6}{|c|}{ Sub-Saharan Africa [41] } \\
\hline Angola & Central African Rep. & Gabon & Madagascar & Niger & South Africa \\
\hline Benin & Chad & Gambia & Malawi & Republic of Congo & Sudan \\
\hline Botswana & Comoro Is. & Ghana & Mali & Rwanda & Swaziland \\
\hline Burkina Faso & Cote d'Ivoire & Guinea & Mauritania & Senegal & Togo \\
\hline Burundi & Equatorial Guinea & Guinea-Bissau & Mauritius & Seychelles & Zaire \\
\hline Cameroon & Eritrea & Lesotho & Mozambique & Sierra Leone & Zambia \\
\hline Cape Verde & Ethiopia & Liberia & Namibia & Somalia & \\
\hline
\end{tabular}

*Excludes high income countries. 
Table 3. Proportion of countries with explicit deposit insurance to total by category (in percent, as of 2003)

\begin{tabular}{lccc}
\hline & \multicolumn{3}{c}{ Proportion based on } \\
Category & Number of countries & GDP & GDP per capita \\
\hline By income level & 75.00 & 96.35 & 83.45 \\
$\quad$ High income & 60.71 & 86.20 & 63.26 \\
Upper middle income & 58.82 & 57.56 & 64.25 \\
$\quad$ Lower middle income & 16.39 & 78.11 & 17.26 \\
Low income & & & \\
By geographical region* & & & \\
Asia \& Pacific & 38.46 & 48.76 & 53.78 \\
Europe \& Central Asia & 74.07 & 97.24 & 93.40 \\
Latin America \& Caribbean & 66.67 & 98.34 & 71.11 \\
Middle East \& North Africa & 28.57 & 16.36 & 42.84 \\
Sub-Saharan Africa & 10.87 & 17.12 & 3.63 \\
\hline
\end{tabular}

*Regional breakdown excludes high income countries

Table 4. Explicit deposit insurance schemes which extend coverage to inter-bank deposits by income level (as of 2003)

\begin{tabular}{llll}
\hline High income & Upper middle income & Lower middle income & Low income \\
\hline Canada & Lebanon & Bosnia-Herzegovina & Kenya \\
United States & & Colombia & Nigeria \\
& & Guatemala & \\
& Honduras & \\
& Marshall Islands & \\
& Micronesia & \\
& Philippines & \\
& Thailand & \\
\hline
\end{tabular}


Table 5. Explicit deposit insurance schemes with co-insurance by income level (co-insurance requirements in parentheses, as of 2003)

\begin{tabular}{lll}
\hline High income & Upper middle income & Lower middle income \\
\hline Austria $(10 \%)$ & Chile $(10 \%)$ & Albania $(15 \%)$ \\
Belgium $(10 \%)$ & Czech Rep. $(10 \%)$ & Belarus $(20 \%)$ \\
Cyprus $(10 \%)$ & Estonia $(10 \%)$ & Bolivia $(50 \%)$ \\
Germany $(10 \%)$ & Lithuania $(10 \%)$ & Colombia $(25 \%)$ \\
Ireland $(10 \%)$ & Oman $(25 \%)$ & Macedonia $(10 \%)$ \\
Isle of Man $(25 \%)$ & Poland (10\%) & Russia $(50 \%)$ \\
Luxembourg $(10 \%)$ & Slovak Republic $(10 \%)$ & \\
United Kingdom $(10 \%)$ & & \\
\hline
\end{tabular}

Table 6. Explicit deposit insurance schemes with risk adjusted premiums by income level

(as of 2003)

\begin{tabular}{lll}
\hline High income & Upper middle income & Lower middle income \\
\hline Finland & Argentina & Belarus \\
Italy & Hungary & Bolivia \\
Portugal & Uruguay & Bulgaria \\
Sweden & & El Salvador \\
Taiwan & & Kazakhstan \\
United States & & Macedonia \\
& & Marshall Islands \\
& Micronesia \\
& & Peru \\
& & Romania \\
\hline
\end{tabular}




\section{Table 7. Design features of explicit deposit insurance schemes}

(as of 2003)

Panel A: Number of countries with each feature in a given category

7.A.1. By level of income

\begin{tabular}{lccccc}
\hline Feature & High income & $\begin{array}{c}\text { Upper middle } \\
\text { income }\end{array}$ & $\begin{array}{c}\text { Lower middle } \\
\text { income }\end{array}$ & Low income & $\begin{array}{c}\text { Total number } \\
\text { of countries }\end{array}$ \\
\hline Foreign currency deposits covered & 22 & 12 & 23 & 4 & 61 \\
Inter-bank deposits covered & 2 & 1 & 8 & 3 & 14 \\
Co-insurance exists & 8 & 7 & 6 & 0 & 21 \\
Payment per depositor & 23 & 15 & 21 & 7 & 66 \\
Scheme is permanently funded & 19 & 15 & 28 & 7 & 69 \\
Premiums are risk-adjusted & 6 & 3 & 11 & 0 & 20 \\
Membership is compulsory & 28 & 16 & 23 & 7 & 74 \\
Source of funding & 15 & 1 & & & 28 \\
$\quad$ Private & 15 & 13 & 11 & 1 & 49 \\
$\quad$ Joint & 0 & 1 & 0 & 6 & 1 \\
$\quad$ Public & 14 & 10 & 19 & 6 & 49 \\
Administration & 9 & 5 & 7 & 1 & 22 \\
$\quad$ Official & 7 & 1 & 1 & 1 & 10 \\
\hline$\quad$ Joint & & & & & \\
\hline
\end{tabular}

7.A.2. By region*

\begin{tabular}{lccccc} 
Feature & $\begin{array}{c}\text { Asia \& } \\
\text { Pacific }\end{array}$ & $\begin{array}{c}\text { Europe \& } \\
\text { Central Asia }\end{array}$ & $\begin{array}{c}\text { Latin America \& } \\
\text { Caribbean }\end{array}$ & $\begin{array}{c}\text { Middle East \& } \\
\text { North Africa }\end{array}$ & $\begin{array}{c}\text { Sub-Saharan } \\
\text { Africa }\end{array}$ \\
\hline Foreign currency deposits covered & 5 & 19 & 12 & 1 & 2 \\
Inter-bank deposits covered & 4 & 1 & 3 & 1 & 3 \\
Co-insurance exists & 0 & 9 & 3 & 1 & 0 \\
Payment per depositor & 6 & 16 & 14 & 3 & 4 \\
Scheme is permanently funded & 6 & 20 & 16 & 4 & 4 \\
Premiums are risk-adjusted & 2 & 7 & 5 & 0 & 0 \\
Membership is compulsory & 3 & 19 & 16 & 4 & 4 \\
Source of funding & & & & & \\
$\quad$ Private & 2 & 5 & 5 & 1 & 0 \\
$\quad$ Joint & 4 & 14 & 9 & 3 & 0 \\
$\quad$ Public & 0 & 0 & 1 & 0 & 3 \\
Administration & & & & & 1 \\
$\quad$ Official & 6 & 12 & 12 & 2 & 1 \\
$\quad$ Joint & 0 & 7 & 3 & 2 & \\
$\quad$ Private & 0 & 0 & 2 & 0 & \\
\hline
\end{tabular}

*Regional breakdown excludes high income countries 


\section{Table 7. Design features of explicit deposit insurance schemes}

(as of 2003, continued)

Panel B: Proportion of countries with each feature in a given category (in percent)

7.B.1. By level of income

\begin{tabular}{lccccc}
\hline Feature & High income & $\begin{array}{c}\text { Upper middle } \\
\text { income }\end{array}$ & $\begin{array}{c}\text { Lower middle } \\
\text { income }\end{array}$ & Low income & $\begin{array}{c}\text { Proportion in } \\
\text { all countries }\end{array}$ \\
\hline Foreign currency deposits covered & 73 & 80 & 82 & 57 & 76 \\
Inter-bank deposits covered & 7 & 7 & 29 & 43 & 18 \\
Co-insurance exists & 27 & 44 & 21 & 0 & 25 \\
Payment per depositor & 77 & 94 & 72 & 78 & 79 \\
Scheme is permanently funded & 63 & 94 & 97 & 100 & 84 \\
Premiums are risk-adjusted & 20 & 19 & 39 & 0 & 25 \\
Membership is compulsory & 93 & 100 & 82 & 100 & 91 \\
Source of funding & & & & & 36 \\
$\quad$ Private & 50 & 7 & 42 & 14 & 63 \\
$\quad$ Joint & 50 & 87 & 58 & 86 & 1 \\
$\quad$ Public & 0 & 7 & 0 & 0 & 60 \\
Administration & & & & & 27 \\
$\quad$ Official & 47 & 63 & 70 & 75 & 12 \\
$\quad$ Joint & 30 & 31 & 26 & 13 & 13 \\
$\quad$ Private & 23 & 6 & 4 & &
\end{tabular}

7.B.2. By region*

\begin{tabular}{lccccc} 
Feature & $\begin{array}{c}\text { Asia \& } \\
\text { Pacific }\end{array}$ & $\begin{array}{c}\text { Europe \& } \\
\text { Central Asia }\end{array}$ & $\begin{array}{c}\text { Latin America \& } \\
\text { Caribbean }\end{array}$ & $\begin{array}{c}\text { Middle East \& } \\
\text { North Africa }\end{array}$ & $\begin{array}{c}\text { Sub-Saharan } \\
\text { Africa }\end{array}$ \\
\hline Foreign currency deposits covered & 71 & 100 & 75 & 25 & 40 \\
Inter-bank deposits covered & 57 & 5 & 19 & 25 & 75 \\
Co-insurance exists & 0 & 45 & 18 & 25 & 0 \\
Payment per depositor & 75 & 80 & 82 & 75 & 80 \\
Scheme is permanently funded & 86 & 100 & 94 & 100 & 100 \\
Premiums are risk-adjusted & 33 & 35 & 29 & 0 & 0 \\
Membership is compulsory & 50 & 95 & 94 & 100 & 100 \\
Source of funding & & & & & 0 \\
$\quad$ Private & 33 & 26 & 33 & 25 & 100 \\
$\quad$ Joint & 67 & 74 & 60 & 75 & 0 \\
$\quad$ Public & 0 & 0 & 7 & 0 & 60 \\
Administration & & & & & 20 \\
$\quad$ Official & 100 & 63 & 71 & 50 & 20 \\
\hline Joint & 0 & 37 & 18 & 50 & \\
\hline
\end{tabular}

*Regional breakdown excludes high income countries 


\section{Table 7. Design features of explicit deposit insurance schemes}

(as of 2003, continued)

Panel C: The sum of the GDP of countries with each feature divided by the total GDP in a given category (in percent)

\section{C.1. By level of income}

\begin{tabular}{|c|c|c|c|c|c|}
\hline Feature & High income & $\begin{array}{c}\text { Upper middle } \\
\text { income }\end{array}$ & $\begin{array}{c}\text { Lower middle } \\
\text { income }\end{array}$ & Low income & $\begin{array}{c}\text { Proportion in } \\
\text { all countries }\end{array}$ \\
\hline Foreign currency deposits covered & 71 & 92 & 46 & 85 & 71 \\
\hline Inter-bank deposits covered & 36 & 1 & 23 & 8 & 34 \\
\hline Co-insurance exists & 18 & 31 & 27 & 0 & 18 \\
\hline Payment per depositor & 60 & 79 & 43 & 87 & 60 \\
\hline Scheme is permanently funded & 80 & 93 & 92 & 100 & 81 \\
\hline Premiums are risk-adjusted & 42 & 27 & 19 & 0 & 39 \\
\hline Membership is compulsory & 97 & 100 & 97 & 100 & 98 \\
\hline \multicolumn{6}{|l|}{ Source of funding } \\
\hline Private & 24 & 21 & 62 & 0 & 25 \\
\hline Joint & 76 & 72 & 38 & 100 & 74 \\
\hline Public & 0 & 7 & 0 & 0 & 0 \\
\hline \multicolumn{6}{|l|}{ Administration } \\
\hline Official & 49 & 56 & 38 & 98 & 50 \\
\hline Joint & 36 & 23 & 12 & 1 & 34 \\
\hline Private & 15 & 21 & 50 & 1 & 17 \\
\hline
\end{tabular}

7.C.2. By region*

\begin{tabular}{|c|c|c|c|c|c|}
\hline Feature & $\begin{array}{l}\text { Asia \& } \\
\text { Pacific }\end{array}$ & $\begin{array}{c}\text { Europe \& } \\
\text { Central Asia } \\
\end{array}$ & $\begin{array}{c}\text { Latin America \& } \\
\text { Caribbean }\end{array}$ & $\begin{array}{c}\text { Middle East \& } \\
\text { North Africa } \\
\end{array}$ & $\begin{array}{c}\text { Sub-Saharan } \\
\text { Africa }\end{array}$ \\
\hline Foreign currency deposits covered & 92 & 100 & 47 & 17 & 29 \\
\hline Inter-bank deposits covered & 32 & 1 & 7 & 15 & 85 \\
\hline Co-insurance exists & 0 & 63 & 10 & 17 & 0 \\
\hline Payment per depositor & 74 & 81 & 43 & 42 & 49 \\
\hline Scheme is permanently funded & 79 & 100 & 95 & 100 & 100 \\
\hline Premiums are risk-adjusted & 0 & 30 & 19 & 0 & 0 \\
\hline Membership is compulsory & 97 & 98 & 99 & 100 & 100 \\
\hline \multicolumn{6}{|l|}{ Source of funding } \\
\hline Private & 0 & 10 & 64 & 10 & 0 \\
\hline Joint & 100 & 90 & 32 & 90 & 100 \\
\hline Public & 0 & 0 & 5 & 0 & 0 \\
\hline \multicolumn{6}{|l|}{ Administration } \\
\hline Official & 100 & 58 & 39 & 27 & 79 \\
\hline Joint & 0 & 42 & 5 & 73 & 10 \\
\hline Private & 0 & 0 & 56 & 0 & 11 \\
\hline
\end{tabular}

*Regional breakdown excludes high income countries 


\title{
Table 7. Design features of explicit deposit insurance schemes
}

\author{
(as of 2003, continued)
}

Panel D: The sum of the GDP per capita of countries with each feature divided by the total GDP per capita in a given category (in percent)

7.D.1. By level of income

\begin{tabular}{lccccc}
\hline Feature & High income & $\begin{array}{c}\text { Upper middle } \\
\text { income }\end{array}$ & $\begin{array}{c}\text { Lower middle } \\
\text { income }\end{array}$ & Low income & $\begin{array}{c}\text { Proportion in } \\
\text { all countries }\end{array}$ \\
\hline Foreign currency deposits covered & 76 & 84 & 78 & 60 & 76 \\
Inter-bank deposits covered & 7 & 4 & 27 & 31 & 8 \\
Co-insurance exists & 30 & 45 & 22 & 0 & 30 \\
Payment per depositor & 79 & 91 & 67 & 81 & 79 \\
Scheme is permanently funded & 64 & 93 & 95 & 100 & 69 \\
Premiums are risk-adjusted & 20 & 24 & 41 & 0 & 21 \\
Membership is compulsory & 92 & 100 & 84 & 100 & 92 \\
Source of funding & & & & & 41 \\
$\quad$ Private & 44 & 10 & 46 & 20 & 58 \\
$\quad$ Joint & 56 & 83 & 54 & 80 & 1 \\
$\quad$ Public & 0 & 8 & 0 & 0 & 40 \\
Administration & & & & & 29 \\
$\quad$ Official & 36 & 61 & 64 & 76 & 31 \\
\hline$\quad$ Joint & 29 & 30 & 26 & 17 & 7 \\
\hline
\end{tabular}

7.D.2. By region*

\begin{tabular}{lccccc} 
Feature & $\begin{array}{c}\text { Asia \& } \\
\text { Pacific }\end{array}$ & $\begin{array}{c}\text { Europe \& } \\
\text { Central Asia }\end{array}$ & $\begin{array}{c}\text { Latin America \& } \\
\text { Caribbean }\end{array}$ & $\begin{array}{c}\text { Middle East \& } \\
\text { North Africa }\end{array}$ & $\begin{array}{c}\text { Sub-Saharan } \\
\text { Africa }\end{array}$ \\
\hline Foreign currency deposits covered & 86 & 100 & 66 & 50 & 46 \\
Inter-bank deposits covered & 81 & 3 & 10 & 24 & 68 \\
Co-insurance exists & 0 & 53 & 17 & 50 & 0 \\
Payment per depositor & 65 & 87 & 75 & 87 & 85 \\
Scheme is permanently funded & 68 & 100 & 89 & 100 & 100 \\
Premiums are risk-adjusted & 52 & 32 & 34 & 0 & 0 \\
Membership is compulsory & 33 & 97 & 96 & 100 & 100 \\
Source of funding & & & & & 0 \\
$\quad$ Private & 52 & 15 & 35 & 13 & 100 \\
$\quad$ Joint & 48 & 85 & 53 & 87 & 0 \\
$\quad$ Public & 0 & 0 & 13 & 0 & \\
Administration & & & & & 53 \\
$\quad$ Official & 100 & 55 & 67 & 37 & 31 \\
$\quad$ Joint & 0 & 45 & 10 & 0 & 13 \\
\hline
\end{tabular}

*Regional breakdown excludes high income countries 


\section{APPENDIX}

\section{A.1 The deposit insurance database}

Table A.1.1. Explicit versus implicit deposit insurance

\begin{tabular}{|c|c|c|c|c|c|}
\hline Country name & $\begin{array}{c}\text { Type } \\
\text { explicit }=1 \\
\text { implicit }=0\end{array}$ & Country name & $\begin{array}{c}\text { Type } \\
\text { explicit }=1 \\
\text { implicit }=0 \\
\end{array}$ & Country name & $\begin{array}{c}\text { Type } \\
\text { explicit }=1 \\
\text { implicit }=0\end{array}$ \\
\hline Afghanistan & 0 & Cyprus & 1 & Jamaica & 1 \\
\hline Albania & 1 & Czech Rep. & 1 & Japan & 1 \\
\hline Algeria & 1 & Denmark & 1 & Jordan & 1 \\
\hline Angola & 0 & Djibouti & 0 & Kazakhstan & 1 \\
\hline Argentina & 1 & Dominican Republic & 1 & Kenya & 1 \\
\hline Armenia & 0 & Ecuador & 1 & Kiribati & 0 \\
\hline Australia & 0 & Egypt & 0 & Korea & 1 \\
\hline Austria & 1 & El Salvador & 1 & Kuwait & 0 \\
\hline Azerbaijan & 0 & Equatorial Guinea & 0 & Kyrgyz Republic & 0 \\
\hline Bahamas & 1 & Eritrea & 0 & Laos & 0 \\
\hline Bahrain & 1 & Estonia & 1 & Latvia & 1 \\
\hline Bangladesh & 1 & Ethiopia & 0 & Lebanon & 1 \\
\hline Barbados & 0 & Fiji & 0 & Lesotho & 0 \\
\hline Belarus & 1 & Finland & 1 & Liberia & 0 \\
\hline Belgium & 1 & France & 1 & Libya & 0 \\
\hline Belize & 0 & Gabon & 0 & Liechtenstein & 1 \\
\hline Benin & 0 & Gambia & 0 & Lithuania & 1 \\
\hline Bhutan & 0 & Georgia & 0 & Luxembourg & 1 \\
\hline Bolivia & 1 & Germany & 1 & Macedonia & 1 \\
\hline Bosnia-Herzegovina & 1 & Ghana & 0 & Madagascar & 0 \\
\hline Botswana & 0 & Gibraltar & 1 & Malawi & 0 \\
\hline Brazil & 1 & Greece & 1 & Malaysia & 1 \\
\hline Brunei & 0 & Grenada & 0 & Maldives & 0 \\
\hline Bulgaria & 1 & Guatemala & 1 & Mali & 0 \\
\hline Burkina Faso & 0 & Guinea & 0 & Malta & 1 \\
\hline Burundi & 0 & Guinea-Bissau & 0 & Marshall Islands & 1 \\
\hline Cambodia & 0 & Guyana & 0 & Mauritania & 0 \\
\hline Cameroon & 0 & Haiti & 0 & Mauritius & 0 \\
\hline Canada & 1 & Honduras & 1 & Mexico & 1 \\
\hline Cape Verde & 0 & Hong Kong & 0 & Micronesia & 1 \\
\hline Central African Rep. & 0 & Hungary & 1 & Moldova & 0 \\
\hline Chad & 0 & Iceland & 1 & Mongolia & 0 \\
\hline Chile & 1 & India & 1 & Morocco & 0 \\
\hline China & 0 & Indonesia & 1 & Mozambique & 0 \\
\hline Colombia & 1 & Iran & 0 & Myanmar & 0 \\
\hline Comoro Is. & 0 & Iraq & 0 & Namibia & 0 \\
\hline Costa Rica & 0 & Ireland & 1 & Nepal & 0 \\
\hline Cote d'Ivoire & 0 & Isle of Man & 1 & Netherlands & 1 \\
\hline Croatia & 1 & Israel & 0 & New Zealand & 0 \\
\hline Cuba & 0 & Italy & 1 & Nicaragua & 1 \\
\hline
\end{tabular}




\begin{tabular}{|c|c|c|c|c|c|}
\hline \multicolumn{6}{|c|}{ Table A.1.1 (continued) } \\
\hline Country name & $\begin{array}{c}\text { Type } \\
\text { explicit }=1 \\
\text { implicit }=0\end{array}$ & Country name & $\begin{array}{c}\text { Type } \\
\text { explicit }=1 \\
\text { implicit }=0\end{array}$ & Country name & $\begin{array}{c}\text { Type } \\
\text { explicit }=1 \\
\text { implicit }=0\end{array}$ \\
\hline Niger & 0 & Sierra Leone & 0 & Trinidad \& Tobago & 1 \\
\hline Nigeria & 1 & Singapore & 0 & Tunisia & 0 \\
\hline Norway & 1 & Slovak Republic & 1 & Turkey & 1 \\
\hline Oman & 1 & Slovenia & 1 & Turkmenistan & 1 \\
\hline Pakistan & 0 & Solomon Is. & 0 & Uganda & 1 \\
\hline Panama & 0 & Somalia & 0 & Ukraine & 1 \\
\hline Papua New Guinea & 0 & South Africa & 0 & United Arab Emirates & 0 \\
\hline Paraguay & 1 & Spain & 1 & United Kingdom & 1 \\
\hline Peru & 1 & Sri Lanka & 1 & United States & 1 \\
\hline Philippines & 1 & St. Lucia & 0 & Uruguay & 1 \\
\hline Poland & 1 & Sudan & 0 & Uzbekistan & 0 \\
\hline Portugal & 1 & Suriname & 0 & Vanuatu & 0 \\
\hline Qatar & 0 & Swaziland & 0 & Venezuela & 1 \\
\hline Republic of Congo & 0 & Sweden & 1 & Vietnam & 1 \\
\hline Romania & 1 & Switzerland & 1 & W. Samoa & 0 \\
\hline Russia & 1 & Syria & 0 & Yemen & 0 \\
\hline Rwanda & 0 & Taiwan & 1 & Zaire & 0 \\
\hline Saudi Arabia & 0 & Tajikistan & 0 & Zambia & 0 \\
\hline Senegal & 0 & Tanzania & 1 & Zimbabwe & 1 \\
\hline Serbia \& Montenegro & 1 & Thailand & 1 & & \\
\hline Seychelles & 0 & Togo & 0 & & \\
\hline
\end{tabular}


Table A.1.2. Date of enactment/revision, coverage type and limits

\begin{tabular}{|c|c|c|c|c|c|}
\hline Country name & $\begin{array}{l}\text { Date enacted / } \\
\text { revised }\end{array}$ & $\begin{array}{l}\text { Foreign } \\
\text { currencies } \\
\text { yes }=1 \text { no }=0\end{array}$ & $\begin{array}{c}\text { Inter-bank } \\
\text { deposits } \\
\text { yes }=1 \text { no }=0\end{array}$ & Coverage limits as of 2003 & $\begin{array}{l}\text { Coverage } \\
\text { limits as of } \\
2003 \text { in } \\
\text { US\$ }\end{array}$ \\
\hline$\overline{\text { Albania }}$ & 2002 & 1 & 0 & Lek 700,000 & 6568 \\
\hline Algeria & 1997 & 0 & 0 & 600,000 dinars & 8263 \\
\hline Argentina & $1979 / 1995$ & 1 & 0 & Arg $\$ 30,000$ & 10327 \\
\hline Austria & $1979 / 1996$ & 1 & 0 & EUR 20,000 & 25260 \\
\hline Bahamas & 1999 & 0 & 0 & 50,000 Bahamian dollars & 50000 \\
\hline Bahrain & 1993 & 1 & 0 & BD 15,000 & 39894 \\
\hline Bangladesh & 1984 & 0 & 0 & Tk 60,000 & 1021 \\
\hline Belarus & $\begin{array}{c}1996 / 1998 / 2000 / \\
2001 / 2004\end{array}$ & 1 & 0 & RBL 2,141,000 & 1000 \\
\hline Belgium & 1974/1995/1998 & 1 & 0 & EUR 20,000 & 25260 \\
\hline Bolivia & 2001 & 1 & 0 & $\begin{array}{l}50 \% \text { of privileged obligations. No } \\
\text { maximum amount. }\end{array}$ & \\
\hline Bosnia-Herzegovina & 1998 & 1 & 1 & 5000 Convertible Markas & 3228 \\
\hline Brazil & $1995 / 2002$ & 0 & 0 & Reais 20,000 & 6925 \\
\hline Bulgaria & $\begin{array}{c}1996 / 1998 / 2001 / \\
2002\end{array}$ & 1 & 0 & BGN 15,000 & 9686 \\
\hline Canada & 1967 & 0 & 1 & Can. $\$ 60,000$ & 46425 \\
\hline Chile & 1986 & 1 & 0 & $\begin{array}{l}\text { demand deposits in full and } 90 \% \\
\text { coinsurance up to UF } 120 \text { for } \\
\text { savings deposits }\end{array}$ & 3764 \\
\hline Colombia & 1985 & 0 & 1 & $75 \%$ per deposit or Col\$ 20 Mil. & 7192 \\
\hline Croatia & 1997 & 1 & 0 & HRV 100,000 & 16343 \\
\hline Cyprus & 2000 & 0 & 0 & EUR 20,000 & 25260 \\
\hline Czech Rep. & 1994 & 1 & 0 & $90 \%$ of EUR 25,000 & 31575 \\
\hline Denmark & $1987 / 1995$ & 1 & 0 & DKK 300,000 & 40296 \\
\hline Dominican Republic & 1962 & 1 & 0 & Full & Full \\
\hline Ecuador & 1998 & 1 & 0 & 7416 & 7416 \\
\hline El Salvador & 1999 & 1 & 0 & Colon 58,424 & 4720 \\
\hline Estonia & 1998 & 1 & 0 & EKK 100,000 & 8058 \\
\hline Finland & 1969/1992/1998 & 1 & 0 & FIM 150,000 & 31863 \\
\hline France & 1980/1986/1999 & 1 & 0 & EUR 70,000 & 88410 \\
\hline Germany & 1966/1969/1998 & 1 & 0 & $\begin{array}{c}\text { private: } 30 \% \text { of bank's equity } \\
\text { capital; official coinsurance } 90 \% \\
\text { to EUR } 20,000\end{array}$ & 25260 \\
\hline Gibraltar & 1998 & 1 & 0 & $\begin{array}{c}\text { lesser of } 90 \% \text { coinsurance or EUR } \\
20,000(18,000 \text { pounds })\end{array}$ & 25260 \\
\hline Greece & $1995 / 2000$ & 1 & 0 & EUR 20,000 & 25260 \\
\hline Guatemala & 1999 & 1 & 1 & 20,000 Quetzales & 2487 \\
\hline Honduras & 1999 & 1 & 1 & $\begin{array}{l}\text { Full coverage until } 2002 ; 165000 \\
\text { L/9500 US\$ as of } 2003\end{array}$ & 9297 \\
\hline Hungary & 1993 & 1 & 0 & HUF $3,222,222$ & 14429 \\
\hline Iceland & $1985 / 1996$ & 1 & 0 & ISK 2,091,000 & 29455 \\
\hline India & 1961 & 1 & 0 & Rs 100,000 & 2193 \\
\hline Indonesia & 1998 & & & Blanket guarantee & \\
\hline Ireland & $1989 / 1995$ & 1 & 0 & $90 \%$ of EUR 20,000 & 25260 \\
\hline
\end{tabular}




\begin{tabular}{|c|c|c|c|c|c|}
\hline \multicolumn{6}{|c|}{ Table A.1.2 (continued) } \\
\hline Country name & $\begin{array}{l}\text { Date enacted / } \\
\text { revised }\end{array}$ & $\begin{array}{l}\text { Foreign } \\
\text { currencies } \\
\text { yes }=1 \text { no }=0\end{array}$ & $\begin{array}{l}\text { Inter-bank } \\
\text { deposits } \\
\text { yes }=1 \text { no }=0\end{array}$ & Coverage limits as of 2003 & $\begin{array}{l}\text { Coverage } \\
\text { limits as of } \\
2003 \text { in } \\
\text { US\$ }\end{array}$ \\
\hline Isle of Man & 1991 & 1 & 0 & $\begin{array}{c}\text { lesser of } 15,000 \text { pounds or } 75 \% \text { of } \\
\text { amount deposited }\end{array}$ & 35694 \\
\hline Italy & $1987 / 1996$ & 1 & 0 & ITL 200 Mil. & 130457 \\
\hline Jamaica & 1998 & 1 & 0 & J\$ 300,000 & 4957 \\
\hline Japan & 1971 & 0 & 0 & 10000000 yen & 93371 \\
\hline Jordan & 2000 & 0 & 0 & JD 10,000 & 14104 \\
\hline Kazakhstan & $1999 / 2003$ & 1 & 0 & 400,000 Tenges & 2774 \\
\hline Kenya & 1988 & 1 & 1 & K Sh 100,000 & 1313 \\
\hline Korea & 1996 & 0 & 0 & 50 Mil Won & 41925 \\
\hline Latvia & 1998 & 1 & 0 & 3000 Lat & 5545 \\
\hline Lebanon & 1967 & 0 & 1 & LL 5,000,000 & 3317 \\
\hline Liechtenstein & $1992 / 2003$ & 1 & 0 & EUR 20,000 & 25260 \\
\hline Lithuania & 1996 & 1 & 0 & LTL 45,000 & 16293 \\
\hline Luxembourg & 1989 & 1 & 0 & EUR 20,000 & 25260 \\
\hline Macedonia & $1996 / 2000 / 2002$ & 1 & 0 & EUR 20,000 & 25260 \\
\hline Malaysia & 1998 & & & Blanket guarantee & \\
\hline Malta & 2003 & 0 & 0 & $\begin{array}{c}\text { EUR 20,000, about } 8600 \text { Maltese } \\
\text { lira }\end{array}$ & 25260 \\
\hline Marshall Islands & 1975 & 1 & 1 & US $\$ 100,000$ & 100000 \\
\hline Mexico & 1986/1990/1999 & 1 & 0 & $32,262,340$ Pesos & 2871337 \\
\hline Micronesia & 1963 & 1 & 1 & US\$ 100,000 & 100000 \\
\hline Netherlands & 1978/1996/1998 & 1 & 0 & EUR 20,000 & 25260 \\
\hline Nicaragua & 2001 & 1 & 0 & US\$ 20,000 & 20000 \\
\hline Nigeria & $1988 / 1989$ & 0 & 1 & N 50,000 & 366 \\
\hline Norway & $1961 / 1997$ & 1 & 0 & NOK 2,000,000 & 299401 \\
\hline Oman & 1995 & 1 & 0 & $\begin{array}{c}\text { RO } 20,000 \text { or } 75 \% \text { of net deposits, } \\
\text { whichever is less }\end{array}$ & 52016 \\
\hline Paraguay & 2003 & n.a. & n.a. & $75 *$ monthly minimum salary & 10500 \\
\hline Peru & 1991 & 1 & 0 & S 68,474 & 19773 \\
\hline Philippines & 1963 & 1 & 1 & P 100,000 & 1800 \\
\hline Poland & 1995 & 1 & 0 & $\begin{array}{l}100 \% \text { of up to EUR } 1,000 ; 90 \% \text { of } \\
\text { EUR } 1,000 \text { to EUR } 22,500\end{array}$ & 28418 \\
\hline Portugal & $1992 / 1995$ & 1 & 0 & EUR 25,000 & 31575 \\
\hline Romania & 1996 & 1 & 0 & ROL $125,222,000$ & 3842 \\
\hline Russia & 2003 & n.a. & n.a. & 100,000 rubles & 3395 \\
\hline Serbia \& Montenegro & 2001 & 1 & 0 & 5,000 Dinars & 87 \\
\hline Slovak Republic & $1996 / 2001$ & 1 & 0 & $90 \%$, not to exceed EUR 20,000 & 25260 \\
\hline Slovenia & 2001 & 1 & 0 & $5,100,000$ tolars & 26931 \\
\hline Spain & $1977 / 1996$ & 1 & 0 & EUR 20,000 & 25260 \\
\hline Sri Lanka & 1987 & 0 & 0 & Rs. 100,000 & 1034 \\
\hline Sweden & 1996 & 1 & 0 & SEK 250,000 & 34364 \\
\hline Switzerland & $1984 / 1993$ & 0 & 0 & CHF 30,000 & 24254 \\
\hline Taiwan & 1985 & 0 & 0 & NT\$ 1,000,000 since Aug 151987 & \\
\hline Tanzania & 1994 & 1 & 1 & TZS 250,000 & 235 \\
\hline Thailand & 1997 & 1 & 1 & $\begin{array}{l}\text { Full coverage (blanket } \\
\text { government guarantee since 1997) }\end{array}$ & Full \\
\hline
\end{tabular}




\begin{tabular}{|c|c|c|c|c|c|}
\hline \multicolumn{6}{|c|}{ Table A.1.2 (continued) } \\
\hline Country name & $\begin{array}{l}\text { Date enacted / } \\
\text { revised }\end{array}$ & $\begin{array}{c}\text { Foreign } \\
\text { currencies } \\
\text { yes }=1 \text { no }=0\end{array}$ & $\begin{array}{c}\text { Inter-bank } \\
\text { deposits } \\
\text { yes }=1 \text { no }=0\end{array}$ & Coverage limits as of 2003 & $\begin{array}{c}\text { Coverage } \\
\text { limits as of } \\
2003 \text { in } \\
\text { US\$ } \\
\end{array}$ \\
\hline Trinidad \& Tobago & 1986 & 0 & 0 & TT $\$ 50,000$ & 7937 \\
\hline Turkey & $1983 / 2000$ & 1 & 0 & unlimited & Full \\
\hline Turkmenistan & 2000 & 1 & 0 & full & Full \\
\hline Uganda & 1994 & 0 & 0 & U Sh $3,000,000$ & 1550 \\
\hline Ukraine & 1998 & 1 & 0 & UAH 1,500 & 281 \\
\hline United Kingdom & $1982 / 1995$ & 1 & 0 & $\begin{array}{l}100 \% \text { of first } £ 2000 \text { and } 90 \% \text { of } \\
\text { next } £ 33,000\end{array}$ & 19611 \\
\hline United States & $1934 / 1991$ & 1 & 1 & US\$ 100,000 & 100000 \\
\hline Uruguay & 2002 & n.a. & n.a. & & \\
\hline Venezuela & $1985 / 2001$ & 0 & 0 & Bs $10,000,000$ & 6258 \\
\hline Vietnam & 2000 & n.a. & n.a. & VND 30,000,000 & 1948 \\
\hline Zimbabwe & 2003 & & & Zimbabwe $\$ 200,000$ & 3640 \\
\hline
\end{tabular}

Notes: Blank spaces indicate that the data is not available.

n.a. stands for "Not applicable" 
Table A.1.3. Co-insurance, payment coverage type, and coverage value distribution

\begin{tabular}{|c|c|c|c|c|c|c|}
\hline Country name & $\begin{array}{c}\text { Co- } \\
\text { insurance } \\
\text { yes }=1 \text { no }=0\end{array}$ & $\begin{array}{c}\text { Co- } \\
\text { insurance } \\
\text { percentage }\end{array}$ & $\begin{array}{c}\text { Payment } \\
\text { per depositor }=1 \\
\text { per deposit }=0\end{array}$ & $\begin{array}{c}\text { Percentage of } \\
\text { deposit value } \\
\text { covered }\end{array}$ & $\begin{array}{l}\text { Information source } \\
\text { for coverage } \\
\text { distribution }\end{array}$ & $\begin{array}{c}\text { Reference date of } \\
\text { data on coverage } \\
\text { distribution }\end{array}$ \\
\hline Albania & 1 & 15 & 1 & & & \\
\hline Algeria & 0 & 0 & 0 & & & \\
\hline Argentina & 0 & 0 & 0 & 40 & Garcia & 2000 \\
\hline Austria & 1 & 10 & 0 & & & \\
\hline Bahamas & 0 & 0 & 0 & 39 & IADI & 2001 \\
\hline Bahrain & 0 & 0 & 0 & & & \\
\hline Bangladesh & 0 & 0 & 0 & 31 & Garcia & 2000 \\
\hline Belarus & 1 & 20 & 0 & & & \\
\hline Belgium & 1 & 10 & 0 & & & \\
\hline Bolivia & 1 & 50 & 0 & & & \\
\hline Bosnia-Herzegovina & 0 & 0 & 0 & & & \\
\hline Brazil & 0 & 0 & 0 & 23.6 & FGC & 2003 \\
\hline Bulgaria & 0 & 0 & 1 & 71 & DIF & 2003 \\
\hline Canada & 0 & 0 & 0 & 34.3 & CDIC & 2002 \\
\hline Chile & 1 & 10 & 1 & 30 & $\begin{array}{c}\text { Demirguc-Kunt and } \\
\text { Levine }\end{array}$ & 2003 \\
\hline Colombia & 1 & 25 & 1 & 30.8 & IADI & 2002 \\
\hline Croatia & 0 & 0 & 1 & 68 & Garcia & 2000 \\
\hline Cyprus & 1 & 10 & 1 & & & \\
\hline Czech Rep. & 1 & 10 & 1 & 86.2 & FPV & 2003 \\
\hline Denmark & 0 & 0 & 1 & 44.6 & IADI & 2001 \\
\hline Dominican Republic & & & & 100 & & 2003 \\
\hline Ecuador & 0 & 0 & 1 & & & \\
\hline El Salvador & 0 & 0 & 1 & & & \\
\hline Estonia & 1 & 10 & 1 & & & \\
\hline Finland & 0 & 0 & 1 & 40 & Garcia & 2000 \\
\hline France & 0 & 0 & 1 & & & \\
\hline Germany & 1 & 10 & 1 & & & \\
\hline Gibraltar & 1 & 10 & 1 & & & \\
\hline Greece & 0 & 0 & 1 & & & \\
\hline Guatemala & 0 & 0 & 1 & & & \\
\hline Honduras & 0 & 0 & 1 & & & \\
\hline Hungary & 0 & 0 & 1 & 86.8 & NDIF & 2003 \\
\hline Iceland & 0 & 0 & 1 & & & \\
\hline India & 0 & 0 & 1 & 72 & Garcia & 2000 \\
\hline \multicolumn{7}{|l|}{ Indonesia } \\
\hline Ireland & 1 & 10 & 1 & & & \\
\hline Isle of Man & 1 & 25 & 1 & & & \\
\hline Italy & 0 & 0 & 1 & 62 & Garcia & 2000 \\
\hline Jamaica & 0 & 0 & 1 & 33.5 & Garcia & 2000 \\
\hline Japan & 0 & 0 & 1 & 87.9 & DIC & 2002 \\
\hline Jordan & 0 & 0 & 1 & & & \\
\hline Kazakhstan & 0 & 0 & 1 & & & \\
\hline Kenya & 0 & 0 & 1 & 16 & Garcia & 2000 \\
\hline Korea & 0 & 0 & 1 & 80.8 & KDIC & 2003 \\
\hline
\end{tabular}


Table A.1.3 (continued)

\begin{tabular}{|c|c|c|c|c|c|c|}
\hline Country name & $\begin{array}{c}\text { Co- } \\
\text { insurance } \\
\text { yes }=1 \text { no }=0\end{array}$ & $\begin{array}{c}\text { Co- } \\
\text { insurance } \\
\text { percentage }\end{array}$ & $\begin{array}{c}\text { Payment } \\
\text { per depositor }=1 \\
\text { per deposit }=0\end{array}$ & $\begin{array}{c}\text { Percentage of } \\
\text { deposit value } \\
\text { covered }\end{array}$ & $\begin{array}{c}\text { Information source } \\
\text { for coverage } \\
\text { distribution }\end{array}$ & $\begin{array}{c}\text { Reference date of } \\
\text { data on coverage } \\
\text { distribution }\end{array}$ \\
\hline Latvia & 0 & 0 & 1 & 18.7 & Garcia & 2000 \\
\hline Lebanon & 0 & 0 & 1 & & & \\
\hline Liechtenstein & 0 & 0 & 1 & & & \\
\hline Lithuania & 1 & 10 & 1 & 44 & Garcia & 2000 \\
\hline Luxembourg & 1 & 10 & 1 & & & \\
\hline Macedonia & 1 & 10 & 1 & 53.7 & IADI/NBRM & 2002 \\
\hline \multicolumn{7}{|l|}{ Malaysia } \\
\hline Malta & 0 & 0 & 1 & & & \\
\hline Marshall Islands & 0 & 0 & 1 & & & \\
\hline Mexico & 0 & 0 & 1 & 81 & IADI/IPAB & 2003 \\
\hline Micronesia & 0 & 0 & 1 & & & \\
\hline Netherlands & 0 & 0 & 1 & & & \\
\hline Nicaragua & 0 & 0 & 1 & & & \\
\hline Nigeria & 0 & 0 & 0 & 19 & NDIC & 2003 \\
\hline Norway & 0 & 0 & 1 & 76.1 & Garcia & 2000 \\
\hline Oman & 1 & 25 & 1 & & & \\
\hline Paraguay & 0 & 0 & 1 & & & \\
\hline Peru & 0 & 0 & 1 & 39.5 & IADI & 2002 \\
\hline Philippines & 0 & 0 & 1 & 19 & PDIC & 2002 \\
\hline Poland & 1 & 10 & 1 & & & \\
\hline Portugal & 0 & 0 & 1 & 53 & FGD & 2002 \\
\hline Romania & 0 & 0 & 1 & 43 & BDGF & 2002 \\
\hline Russia & 1 & 50 & 1 & 85 & WB & 2003 \\
\hline Serbia \& Montenegro & 0 & 0 & 1 & & & \\
\hline Slovak Republic & 1 & 10 & 1 & 47 & Garcia & 2000 \\
\hline Slovenia & 0 & 0 & 1 & & & \\
\hline Spain & 0 & 0 & 1 & 60 & FGD & 2000 \\
\hline Sri Lanka & 0 & 0 & 1 & & & \\
\hline Sweden & 0 & 0 & 1 & 57 & IADI & 2002 \\
\hline Switzerland & 0 & 0 & 1 & & & \\
\hline Taiwan & 0 & 0 & 0 & 35.8 & IADI & 2001 \\
\hline Tanzania & 0 & 0 & 1 & 12 & Garcia & 2000 \\
\hline Thailand & 0 & 0 & 0 & 100 & & 2003 \\
\hline Trinidad \& Tobago & 0 & 0 & 1 & 34.1 & Garcia & 2000 \\
\hline Turkey & 0 & 0 & 0 & 100 & & 2003 \\
\hline Turkmenistan & 0 & 0 & 0 & 100 & & 2003 \\
\hline Uganda & 0 & 0 & 1 & 26 & Garcia & 2000 \\
\hline Ukraine & 0 & 0 & 1 & 19 & Garcia & 2000 \\
\hline United Kingdom & 1 & 10 & 1 & & & \\
\hline United States & 0 & 0 & 0 & $65 / 60$ & Garcia/FDIC & $2000 / 2003$ \\
\hline Uruguay & 0 & 0 & 1 & & & \\
\hline Venezuela & 0 & 0 & 1 & & & \\
\hline Vietnam & 0 & 0 & 1 & & & \\
\hline Zimbabwe & 0 & 0 & 1 & & & \\
\hline
\end{tabular}

Notes: Blank spaces indicate that the data is not available.

n.a. stands for "Not applicable" 
Table A.1.4. Type of fund and premium information

\begin{tabular}{|c|c|c|c|c|}
\hline Country name & $\begin{array}{c}\text { Permanent } \\
\text { fund } \\
\text { funded }=1 \\
\text { unfunded }=0 \\
\end{array}$ & $\begin{array}{l}\text { Premium or } \\
\text { assessment base }\end{array}$ & $\begin{array}{l}\text { Annual premiums } \\
\% \text { of base }\end{array}$ & $\begin{array}{c}\text { Risk- } \\
\text { adjusted } \\
\text { premiums } \\
\text { yes }=1 \mathrm{no}=0 \\
\end{array}$ \\
\hline Albania & 1 & insured deposits & $0.50 \%$ & 0 \\
\hline Algeria & 1 & & & 0 \\
\hline Argentina & 1 & insured deposits & risk-based, $0.36 \%$ to $0.72 \%$ & 1 \\
\hline Austria & 0 & insured deposits & pro rata, ex post & 0 \\
\hline Bahamas & 1 & insured deposits & $0.05 \%$ & 0 \\
\hline Bahrain & 0 & deposits & ex post & 0 \\
\hline Bangladesh & 1 & deposits & $0.50 \%$ & 0 \\
\hline Belarus & 1 & household deposits & $\begin{array}{l}\text { risk based: } 0 \text { for two state banks. } 0.1 \% \text { to } \\
0.3 \% \text { of household deposits for other banks, } \\
\text { depending on the bank's household deposits } \\
\text { to capital ratio }\end{array}$ & 1 \\
\hline Belgium & 1 & insured liabilities & $0.02 \%+0.04 \%$ & 0 \\
\hline Bolivia & 1 & deposits & & 1 \\
\hline Bosnia-Herzegovina & 1 & deposits & $0.5 \%$ until July 2001, then changed to $0.3 \%$ & 0 \\
\hline Brazil & 1 & insured deposits & $0.30 \%$ & 0 \\
\hline Bulgaria & 1 & insured deposits & risk based to $0.5 \%$ & 1 \\
\hline Canada & 1 & insured deposits & $0.33 \% \max$ & 0 \\
\hline Chile & 0 & not applicable & none & 0 \\
\hline Colombia & 1 & insured deposits & $0.5 \%$ from January 2002 to December 2006 & 0 \\
\hline Croatia & 1 & insured deposits & $0.80 \%$ & 0 \\
\hline Cyprus & 1 & n.a. & n.a. & 0 \\
\hline Czech Rep. & 1 & insured deposits & $0.10 \%$ & 0 \\
\hline Denmark & 1 & insured deposits & $0.2 \%$ (maximum) & 0 \\
\hline Dominican Republic & 1 & deposits & $0.1875 \%$ & 0 \\
\hline Ecuador & 1 & deposits & $0.65 \%$ & 0 \\
\hline El Salvador & 1 & insured deposits & risk-based, $0.1 \%$ to $0.3 \%$ & 1 \\
\hline Estonia & 1 & deposits until 2002 & $0.5 \%$ (maximum) ( $0.28 \%$ at present) & 0 \\
\hline Finland & 1 & insured deposits & risk based: $0.05 \%$ to $0.3 \%$ & 1 \\
\hline France & 0 & n.a. & on demand but limited & 0 \\
\hline Germany & 1 & $\begin{array}{l}\text { insured deposits in } \\
\text { commercial banks } \\
\text { DIS, risk-assets in } \\
\text { other DIS }\end{array}$ & official is $0.03 \%$ but can be doubled & 0 \\
\hline Gibraltar & 0 & insured deposits & $\begin{array}{l}\text { administrative expenses and expost } \\
\text { contributions }\end{array}$ & 0 \\
\hline Greece & 1 & deposits & decreasing by size: $1.25 \%$ to $0.025 \%$ & 0 \\
\hline Guatemala & 1 & insured deposits & $\begin{array}{l}1.0 \% \text { plus } 0.5 \% \text { when the fund falls below its } \\
\text { target }\end{array}$ & 0 \\
\hline Honduras & 1 & deposits & not more than $0.25 \%$ & 0 \\
\hline Hungary & 1 & insured deposits & risk based to $0.3 \%$ & 1 \\
\hline Iceland & 1 & insured deposits & $0.15 \%$ & 0 \\
\hline India & 1 & deposits & $0.05 \%$ & 0 \\
\hline $\begin{array}{l}\text { Indonesia } \\
\text { Ireland }\end{array}$ & 1 & $\begin{array}{l}\text { EU and EEA, i.e } \\
\text { insured deposits }\end{array}$ & $0.20 \%$ & 0 \\
\hline
\end{tabular}




\begin{tabular}{|c|c|c|c|c|}
\hline \multicolumn{5}{|c|}{ Table A.1.4 (continued) } \\
\hline Country name & $\begin{array}{c}\text { Permanent } \\
\text { fund } \\
\text { funded }=1 \\
\text { unfunded }=0\end{array}$ & $\begin{array}{l}\text { Premium or } \\
\text { assessment base }\end{array}$ & $\begin{array}{l}\text { Annual premiums } \\
\% \text { of base }\end{array}$ & $\begin{array}{c}\text { Risk- } \\
\text { adjusted } \\
\text { premiums } \\
\text { yes }=1 \mathrm{no}=0\end{array}$ \\
\hline Isle of Man & 0 & deposits & $\begin{array}{l}\text { the greater of } 25,000 \text { pounds and } 0.0125 \% \text { of } \\
\text { deposit base subject to a maximum annual } \\
\text { contribution of } 250,000 \text { pounds }\end{array}$ & 0 \\
\hline Italy & 0 & $\begin{array}{l}\text { protected funds } \\
\text { adjusted for size } \\
\text { and risk }\end{array}$ & risk adjusted ex post $0.4 \%$ to $0.8 \%$ & 1 \\
\hline Jamaica & 1 & insured deposits & $0.10 \%$ & 0 \\
\hline Japan & 1 & insured deposits & $0.0048 \%+0.036 \%$ & 0 \\
\hline Jordan & 1 & deposits & $0.20 \%$ & 0 \\
\hline Kazakhstan & 1 & insured deposits & $1.00 \%$ & 1 \\
\hline Kenya & 1 & deposits & $0.15 \%$ & 0 \\
\hline Korea & 1 & deposits & $0.05 \%$ & 0 \\
\hline Latvia & 1 & insured deposits & $0.3 \%$ until year $2000 ; 0.2 \%$ thereafter & 0 \\
\hline Lebanon & 1 & credit accounts & $0.05 \%$ & 0 \\
\hline Liechtenstein & 0 & n.a. & & 0 \\
\hline Lithuania & 1 & insured deposits & $0.45 \%$ & 0 \\
\hline Luxembourg & 0 & insured deposits & ex post & 0 \\
\hline Macedonia & 1 & insured deposits & $1.5 \%$, risk-based $1 \%$ to $5 \%$ & 1 \\
\hline \multicolumn{5}{|l|}{ Malaysia } \\
\hline Malta & 1 & deposits & $0.10 \%$ & 0 \\
\hline Marshall Islands & 1 & deposits & risk-based, $0 \%$ to $0.27 \%$ & 1 \\
\hline Mexico & 1 & all obligations & $\begin{array}{l}\text { minimum } 0.4 \% \text { on a proxy of total bank } \\
\text { liabilities }\end{array}$ & 0 \\
\hline Micronesia & 1 & deposits & risk-based, $0 \%$ to $0.27 \%$ & 1 \\
\hline Netherlands & 0 & case by case & Ex post & 0 \\
\hline Nicaragua & 1 & & & 0 \\
\hline Nigeria & 1 & deposits & $0.94 \%$ & 0 \\
\hline Norway & 1 & $\begin{array}{l}\text { risk-weighted } \\
\text { assets and total } \\
\text { deposits }\end{array}$ & $0.005 \%$ of assets and $0.01 \%$ of total deposits & 0 \\
\hline Oman & 1 & deposits & $0.02 \%$ & 0 \\
\hline Paraguay & n.a. & n.a. & & n.a. \\
\hline Peru & 1 & insured deposits & risk-based from $0.45 \%$ to $1.45 \%$ & 1 \\
\hline Philippines & 1 & deposits & $0.20 \%$ & 0 \\
\hline Poland & 1 & $\begin{array}{l}\text { deposits, also risk- } \\
\text { adjusted assets }\end{array}$ & not more than $0.4 \%$ & 0 \\
\hline Portugal & 1 & insured deposits & $\begin{array}{l}\text { risk-based, } 0.1 \% \text { to } 0.2 \%+\text { more in } \\
\text { emergencies }\end{array}$ & 1 \\
\hline Romania & 1 & insured deposits & risk-based: $0.3 \%$ to $0.6 \%$ & 1 \\
\hline Russia & 1 & deposits & $\begin{array}{l}\text { no more than } 0.15 \% \text {, in emergency up to } \\
0.3 \% \text {, once fund formed } 0.05 \%\end{array}$ & 0 \\
\hline Serbia \& Montenegro & 1 & & & 0 \\
\hline Slovak Republic & 1 & insured deposits & $0.1 \%$ to $0.3 \%$ for banks & 0 \\
\hline Slovenia & 0 & insured deposits & $3.2 \%$ of guaranteed deposits & 0 \\
\hline Spain & 1 & insured deposits & maximum of $0.2 \%$ & 0 \\
\hline Sri Lanka & 1 & deposits & $0.15 \%$ & 0 \\
\hline
\end{tabular}


Table A.1.4 (continued)

\begin{tabular}{|c|c|c|c|c|}
\hline Country name & $\begin{array}{c}\text { Permanent } \\
\text { fund } \\
\text { funded }=1 \\
\text { unfunded }=0\end{array}$ & $\begin{array}{l}\text { Premium or } \\
\text { assessment base }\end{array}$ & $\begin{array}{l}\text { Annual premiums } \\
\% \text { of base }\end{array}$ & $\begin{array}{c}\text { Risk- } \\
\text { adjusted } \\
\text { premiums } \\
\text { yes }=1 \text { no }=0\end{array}$ \\
\hline Sweden & 1 & insured deposits & $\begin{array}{l}\text { risk-based, } 0.5 \% \text { now, } 0.1 \% \text { later (future date } \\
\text { is not available) }\end{array}$ & 1 \\
\hline Switzerland & 0 & balance sheet items & on demand & 0 \\
\hline Taiwan & 1 & insured deposits & $\begin{array}{l}\text { based on three levels of risk: } 0.05 \%, 0.055 \% \text {, } \\
\text { and } 0.06 \%\end{array}$ & 1 \\
\hline Tanzania & 1 & deposits & $0.10 \%$ & 0 \\
\hline Thailand & 0 & & & \\
\hline Trinidad \& Tobago & 1 & deposits & $0.20 \%$ & 0 \\
\hline Turkey & 1 & $\begin{array}{l}\text { insured savings } \\
\text { deposits }\end{array}$ & risk-based $1.0 \%$ to $1.2 \%$ & 1 \\
\hline Turkmenistan & 1 & & & 0 \\
\hline Uganda & 1 & deposits & $0.20 \%$ & 0 \\
\hline Ukraine & 1 & total deposits & 0.5 plus special charges & 0 \\
\hline United Kingdom & 0 & $\begin{array}{l}\text { EEA deposits i.e. } \\
\text { insured deposits }\end{array}$ & on demand & 0 \\
\hline United States & 1 & domestic deposits & risk-based, $0 \%$ to $0.27 \%$ & 1 \\
\hline Uruguay & 1 & & & 1 \\
\hline Venezuela & 1 & insured deposits & $2.00 \%$ & 0 \\
\hline Vietnam & n.a. & & & \\
\hline Zimbabwe & & & & \\
\hline
\end{tabular}

Notes: Blank spaces indicate that the data is not available. n.a. stands for "Not applicable" 
Table A.1.5. Source of funding, administration and membership type

\begin{tabular}{|c|c|c|c|}
\hline Country name & $\begin{array}{l}\text { Source of funding } \\
\text { government }=2 \\
\text { private }=0, \text { joint }=1\end{array}$ & $\begin{array}{c}\text { Administration } \\
\text { private }=3 \text {, joint }=2 \\
\text { official }=1\end{array}$ & $\begin{array}{c}\text { Membership } \\
\text { compulsory }=1 \\
\text { voluntary }=0\end{array}$ \\
\hline Albania & 1 & 1 & 1 \\
\hline Algeria & 1 & 2 & 1 \\
\hline Argentina & 0 & 3 & 1 \\
\hline Austria & 1 & 3 & 1 \\
\hline Bahamas & 0 & 1 & 1 \\
\hline Bahrain & 0 & 2 & 1 \\
\hline Bangladesh & 1 & 1 & 1 \\
\hline Belarus & 0 & 1 & 1 \\
\hline Belgium & 1 & 2 & 1 \\
\hline Bolivia & 0 & 1 & 1 \\
\hline Bosnia-Herzegovina & 0 & 1 & 1 \\
\hline Brazil & 0 & 3 & 1 \\
\hline Bulgaria & 1 & 2 & 1 \\
\hline Canada & 1 & 1 & 1 \\
\hline Chile & 2 & 1 & 1 \\
\hline Colombia & 0 & 1 & 1 \\
\hline Croatia & 1 & 1 & 1 \\
\hline Cyprus & 0 & 2 & 1 \\
\hline Czech Rep. & 1 & 1 & 1 \\
\hline Denmark & 1 & 2 & 1 \\
\hline Dominican Republic & 1 & 2 & 0 \\
\hline Ecuador & n.a. & 1 & 1 \\
\hline El Salvador & 1 & 1 & 1 \\
\hline Estonia & 1 & 2 & 1 \\
\hline Finland & 1 & 3 & 1 \\
\hline France & 0 & 3 & 1 \\
\hline Germany & 0 & 2 & 1 \\
\hline Gibraltar & 0 & 2 & 1 \\
\hline Greece & 0 & 2 & 1 \\
\hline Guatemala & 1 & 1 & 1 \\
\hline Honduras & 1 & 2 & 1 \\
\hline Hungary & 1 & 2 & 1 \\
\hline Iceland & 0 & 1 & 1 \\
\hline India & 1 & 1 & 1 \\
\hline \multicolumn{4}{|l|}{ Indonesia } \\
\hline Ireland & 0 & 1 & 1 \\
\hline Isle of Man & 0 & 1 & 1 \\
\hline Italy & 1 & 3 & 1 \\
\hline Jamaica & 1 & 1 & 1 \\
\hline Japan & 1 & 2 & 1 \\
\hline Jordan & 0 & 1 & 1 \\
\hline Kazakhstan & 0 & 1 & 0 \\
\hline Kenya & 1 & 1 & 1 \\
\hline Korea & 1 & 1 & 1 \\
\hline Latvia & 1 & 1 & 1 \\
\hline Lebanon & 1 & 2 & 1 \\
\hline
\end{tabular}




\begin{tabular}{|c|c|c|c|}
\hline \multicolumn{4}{|c|}{ Table A.1.5 (continued) } \\
\hline Country name & $\begin{array}{c}\text { Source of funding } \\
\text { government }=2 \\
\text { private }=0 \text {, joint }=1\end{array}$ & $\begin{array}{c}\text { Administration } \\
\text { private }=3 \text {, joint }=2 \\
\text { official }=1\end{array}$ & $\begin{array}{c}\text { Membership } \\
\text { compulsory }=1 \\
\text { voluntary }=0\end{array}$ \\
\hline Liechtenstein & 0 & 1 & 1 \\
\hline Lithuania & 1 & 1 & 1 \\
\hline Luxembourg & 0 & 3 & 1 \\
\hline Macedonia & 1 & 3 & 0 \\
\hline \multicolumn{4}{|l|}{ Malaysia } \\
\hline Malta & 0 & 2 & 1 \\
\hline Marshall Islands & 0 & 1 & 0 \\
\hline Mexico & 1 & 1 & 1 \\
\hline Micronesia & 0 & 1 & 0 \\
\hline Netherlands & 1 & 1 & 1 \\
\hline Nicaragua & 0 & 1 & 1 \\
\hline Nigeria & 1 & 1 & 1 \\
\hline Norway & 1 & 3 & 1 \\
\hline Oman & 1 & 1 & 1 \\
\hline Paraguay & n.a. & n.a. & n.a. \\
\hline Peru & 1 & 2 & 1 \\
\hline Philippines & 1 & 1 & 1 \\
\hline Poland & 1 & 2 & 1 \\
\hline Portugal & 1 & 1 & 1 \\
\hline Romania & 1 & 2 & 1 \\
\hline Russia & n.a. & n.a. & 1 \\
\hline Serbia \& Montenegro & 0 & 1 & 1 \\
\hline Slovak Republic & 1 & 2 & 1 \\
\hline Slovenia & 0 & 1 & 1 \\
\hline Spain & 1 & 2 & 1 \\
\hline Sri Lanka & 1 & 1 & 0 \\
\hline Sweden & 1 & 1 & 1 \\
\hline Switzerland & 0 & 3 & 0 \\
\hline Taiwan & 1 & 1 & 0 \\
\hline Tanzania & 1 & 3 & 1 \\
\hline \multicolumn{4}{|l|}{ Thailand } \\
\hline Trinidad \& Tobago & 1 & 1 & 1 \\
\hline Turkey & 1 & 1 & 1 \\
\hline Turkmenistan & 0 & 1 & 1 \\
\hline Uganda & 1 & 1 & 1 \\
\hline Ukraine & 1 & 1 & 1 \\
\hline United Kingdom & 0 & 1 & 1 \\
\hline United States & 1 & 1 & 1 \\
\hline Uruguay & & 1 & 1 \\
\hline Venezuela & 1 & 1 & 1 \\
\hline \multicolumn{4}{|l|}{ Vietnam } \\
\hline Zimbabwe & & 2 & \\
\hline
\end{tabular}

Note: Blank spaces indicate that the data is not available. n.a. stands for "Not applicable" 
Table A.1.6. Barth, Caprio and Levine (2004) survey questions

Panel A: Deposit insurance authority, banking failures, compensation

\begin{tabular}{|c|c|c|c|c|}
\hline Country name & $\begin{array}{l}\text { Does the deposit } \\
\text { insurance } \\
\text { authority make } \\
\text { the decision to } \\
\text { intervene a } \\
\text { bank? } \\
\text { yes }=1 \text { no }=0\end{array}$ & $\begin{array}{c}\text { Does the deposit } \\
\text { insurance authority have } \\
\text { the legal power to cancel } \\
\text { or revoke deposit } \\
\text { insurance for any } \\
\text { participating bank? } \\
\text { yes }=1 \text { no }=0\end{array}$ & $\begin{array}{c}\text { As part of failure } \\
\text { resolution, how } \\
\text { many banks } \\
\text { closed or merged } \\
\text { in the last } 5 \text { years? } \\
\text { number }\end{array}$ & $\begin{array}{c}\text { Were depositors } \\
\text { wholly compensated } \\
\text { (to the extent of } \\
\text { legal protection) the } \\
\text { last time a bank } \\
\text { failed? } \\
\text { yes }=1 \text { no }=0\end{array}$ \\
\hline Albania & 0 & 1 & 0 & No cases \\
\hline Algeria & 1 & 0 & & 0 \\
\hline Argentina & 0 & 0 & 21 & 1 \\
\hline Austria & 0 & 1 & 4 & 1 \\
\hline Bahrain & 0 & 1 & 0 & No cases \\
\hline Belarus & 1 & 1 & 8 & 0 \\
\hline Belgium & 0 & 0 & 2 & 1 \\
\hline Bolivia & & & 1 & 1 \\
\hline Bosnia-Herzegovina & 0 & 1 & 10 & 1 \\
\hline Brazil & 0 & 0 & 18 & 0 \\
\hline Bulgaria & 0 & 0 & 4 & 1 \\
\hline Canada & 1 & 1 & 0 & 0 \\
\hline Chile & 0 & 0 & 0 & No cases \\
\hline Colombia & 0 & 0 & & 0 \\
\hline Croatia & 0 & 0 & 15 & 1 \\
\hline Cyprus & 0 & 1 & 0 & No cases \\
\hline Czech Rep. & 0 & 0 & 9 & 0 \\
\hline Denmark & 0 & 1 & 2 & 0 \\
\hline Ecuador & 0 & 0 & 15 & 0 \\
\hline El Salvador & 0 & 0 & 0 & No cases \\
\hline Estonia & 0 & 0 & 4 & 1 \\
\hline Finland & 0 & 1 & 0 & \\
\hline France & 0 & 1 & 23 & 1 \\
\hline Germany & 0 & 0 & 4 & 1 \\
\hline Gibraltar & 0 & 0 & 0 & No cases \\
\hline Greece & 0 & 1 & 0 & 1 \\
\hline Guatemala & 0 & 0 & 3 & 1 \\
\hline Honduras & 0 & 0 & 3 & 1 \\
\hline Hungary & 1 & 1 & 1 & 1 \\
\hline Iceland & 0 & 0 & 0 & \\
\hline India & 0 & 1 & 40 & 1 \\
\hline Ireland & 0 & 1 & 0 & \\
\hline Isle of Man & 1 & 0 & 0 & 1 \\
\hline Italy & 1 & 1 & 28 & 1 \\
\hline Japan & 0 & 0 & 15 & 1 \\
\hline Jordan & 0 & 0 & 0 & \\
\hline Kazakhstan & 0 & 1 & & 1 \\
\hline Kenya & 0 & 1 & 22 & 1 \\
\hline Korea & 0 & 0 & 15 & 1 \\
\hline Latvia & 0 & 0 & 1 & 0 \\
\hline
\end{tabular}




\begin{tabular}{|c|c|c|c|c|}
\hline \multicolumn{5}{|c|}{ Table A.1.6 Panel A (continued) } \\
\hline Country name & $\begin{array}{c}\text { Does the deposit } \\
\text { insurance } \\
\text { authority make the } \\
\text { decision to } \\
\text { intervene a bank? } \\
\text { yes }=1 \text { no }=0\end{array}$ & $\begin{array}{c}\text { Does the deposit } \\
\text { insurance authority have } \\
\text { the legal power to cancel } \\
\text { or revoke deposit } \\
\text { insurance for any } \\
\text { participating bank? } \\
\text { yes }=1 \text { no }=0\end{array}$ & $\begin{array}{c}\text { As part of failure } \\
\text { resolution, how } \\
\text { many banks } \\
\text { closed or merged } \\
\text { in the last } 5 \text { years? } \\
\text { number }\end{array}$ & $\begin{array}{c}\text { Were depositors } \\
\text { wholly compensated } \\
\text { (to the extent of } \\
\text { legal protection) the } \\
\text { last time a bank } \\
\text { failed? } \\
\text { yes }=1 \text { no }=0\end{array}$ \\
\hline Lebanon & 0 & 0 & 6 & 1 \\
\hline Liechtenstein & 0 & 0 & 0 & \\
\hline Lithuania & 0 & 1 & 4 & 0 \\
\hline Luxembourg & 0 & 1 & 0 & 1 \\
\hline Macedonia & 0 & 0 & 5 & 1 \\
\hline Malaysia & & & 3 & 1 \\
\hline Malta & 0 & 0 & 0 & 0 \\
\hline Mexico & 0 & 0 & & 1 \\
\hline Netherlands & 0 & 0 & 0 & No cases \\
\hline Nicaragua & 0 & 0 & 6 & 1 \\
\hline Nigeria & 0 & 1 & 30 & 0 \\
\hline Norway & 0 & 0 & 0 & 1 \\
\hline Oman & 1 & 1 & 0 & \\
\hline Paraguay & & & 7 & \\
\hline Peru & 0 & 0 & 7 & 1 \\
\hline Philippines & 0 & 1 & 2 & 1 \\
\hline Poland & 0 & 0 & 21 & 1 \\
\hline Portugal & 0 & 0 & 0 & \\
\hline Romania & 0 & 0 & 10 & 1 \\
\hline Serbia \& Montenegro & 0 & 0 & & 1 \\
\hline Slovak Republic & 1 & 0 & 7 & 1 \\
\hline Slovenia & 1 & 0 & 0 & No cases \\
\hline Spain & 0 & 0 & 0 & 1 \\
\hline Sri Lanka & 0 & 1 & & \\
\hline Sweden & 0 & 1 & 0 & \\
\hline Switzerland & 0 & 1 & & 1 \\
\hline Taiwan & 0 & 0 & 0 & \\
\hline Thailand & 0 & 0 & 7 & 1 \\
\hline Trinidad \& Tobago & 0 & 0 & 0 & 1 \\
\hline Turkey & 0 & 0 & 24 & 1 \\
\hline Turkmenistan & 0 & 0 & 2 & 1 \\
\hline Ukraine & 1 & 1 & 46 & 0 \\
\hline United Kingdom & 0 & 0 & & 1 \\
\hline United States & 1 & 1 & & \\
\hline Venezuela & 0 & 0 & 0 & 1 \\
\hline Zimbabwe & & & 2 & 0 \\
\hline
\end{tabular}

Notes: The countries which did not provide answers for any of the survey questions listed here are excluded.

Blank spaces indicate no response. 
Table A.1.6. Barth, Caprio and Levine (2004) survey questions (continued)

Panel B: Depositor compensation processing time and coverage at banking failure

\begin{tabular}{|c|c|c|c|}
\hline Country name & $\begin{array}{l}\text { On average, how } \\
\text { long does it take } \\
\text { to pay depositors } \\
\text { in full? } \\
\text { in months }\end{array}$ & $\begin{array}{l}\text { What was the longest } \\
\text { that depositors had } \\
\text { to wait in the last } 5 \\
\text { years? } \\
\text { in months }\end{array}$ & $\begin{array}{l}\text { Were any deposits not explicitly covered by } \\
\text { deposit insurance at the time of the failure } \\
\text { compensated when the bank failed (excluding } \\
\text { funds later paid out in liquidation } \\
\text { procedures)? } \\
\text { yes }=1 \text { no }=0\end{array}$ \\
\hline Albania & & & No cases \\
\hline Algeria & & & 0 \\
\hline Argentina & & 9 & 1 \\
\hline Austria & 3 & 3 & 0 \\
\hline \multicolumn{4}{|l|}{ Bahrain } \\
\hline Belarus & 24 & & 0 \\
\hline Belgium & 12 & 24 & 0 \\
\hline Bolivia & & 54 & 1 \\
\hline Bosnia-Herzegovina & 6 & 18 & 1 \\
\hline Brazil & 1 & 1 & 0 \\
\hline Bulgaria & 1.5 & 1.5 & 1 \\
\hline Canada & 1 & 0.25 & 0 \\
\hline \multicolumn{4}{|l|}{ Chile } \\
\hline Colombia & 48 & 60 & 0 \\
\hline Croatia & 12 & 24 & 0 \\
\hline \multicolumn{4}{|l|}{ Cyprus } \\
\hline Czech Rep. & 3 & 5 & 1 \\
\hline Denmark & 3 & 3 & 1 \\
\hline Ecuador & & 60 & 1 \\
\hline El Salvador & 2 & & 0 \\
\hline Estonia & 9 & 9 & 0 \\
\hline \multicolumn{4}{|l|}{ Finland } \\
\hline France & & & 1 \\
\hline Germany & & & 1 \\
\hline \multicolumn{4}{|l|}{ Gibraltar } \\
\hline Greece & 3 & & 0 \\
\hline Guatemala & & & 1 \\
\hline Honduras & 0.17 & 0.17 & 1 \\
\hline Hungary & 3 & 3 & 0 \\
\hline \multicolumn{4}{|l|}{ Iceland } \\
\hline India & 5 & 5 & 0 \\
\hline \multicolumn{4}{|l|}{ Ireland } \\
\hline Isle of Man & & & 0 \\
\hline Italy & 0 & 5 & 1 \\
\hline Japan & 0 & 0 & 0 \\
\hline \multicolumn{4}{|l|}{ Jordan } \\
\hline Kazakhstan & 0 & 0 & 1 \\
\hline Kenya & 12 & 18 & 0 \\
\hline Korea & 3 & 6 & 0 \\
\hline Latvia & & & 0 \\
\hline Lebanon & 84 & 120 & 0 \\
\hline
\end{tabular}


Table A.1.6 Panel B (continued)

\begin{tabular}{|c|c|c|c|}
\hline Country name & $\begin{array}{l}\text { On average, how } \\
\text { long does it take } \\
\text { to pay depositors } \\
\text { in full? } \\
\text { in months }\end{array}$ & $\begin{array}{c}\text { What was the longest } \\
\text { that depositors had } \\
\text { to wait in the last } 5 \\
\text { years? } \\
\text { in months }\end{array}$ & $\begin{array}{c}\text { Were any deposits not explicitly covered by } \\
\text { deposit insurance at the time of the failure } \\
\text { compensated when the bank failed (excluding } \\
\text { funds later paid out in liquidation } \\
\text { procedures)? } \\
\text { yes }=1 \text { no }=0\end{array}$ \\
\hline \multicolumn{4}{|l|}{ Liechtenstein } \\
\hline Lithuania & 3 & 6 & 1 \\
\hline Luxembourg & 3 & & 0 \\
\hline Macedonia & & & 0 \\
\hline \multicolumn{4}{|l|}{ Malaysia } \\
\hline Malta & & & 0 \\
\hline \multicolumn{4}{|l|}{ Mexico } \\
\hline Netherlands & 3 & & \\
\hline Nicaragua & & & 0 \\
\hline Nigeria & 36 & 60 & 0 \\
\hline Norway & 1 & 3 & 1 \\
\hline \multicolumn{4}{|l|}{ Oman } \\
\hline Paraguay & 3 & 12 & 0 \\
\hline Peru & 2 & 0.25 & 0 \\
\hline Philippines & 3 & 7 & 0 \\
\hline Poland & 4 & 23 & 0 \\
\hline \multicolumn{4}{|l|}{ Portugal } \\
\hline Romania & 3 & 3 & 0 \\
\hline Serbia \& Montenegro & & & 1 \\
\hline Slovak Republic & 3 & 3 & 0 \\
\hline \multicolumn{4}{|l|}{ Slovenia } \\
\hline Spain & 3 & 3 & 0 \\
\hline \multicolumn{4}{|l|}{ Sri Lanka } \\
\hline \multicolumn{4}{|l|}{ Sweden } \\
\hline \multicolumn{4}{|l|}{ Switzerland } \\
\hline Taiwan & & & 1 \\
\hline Thailand & 1 & 4 & 0 \\
\hline Trinidad \& Tobago & 2 & & 0 \\
\hline Turkey & 3 & & 1 \\
\hline Turkmenistan & 2 & 1 & 0 \\
\hline Ukraine & 60 & 60 & 0 \\
\hline United Kingdom & 6 & 6 & 0 \\
\hline United States & 0.1 & & \\
\hline Venezuela & & & 0 \\
\hline Zimbabwe & 6 & & \\
\hline
\end{tabular}

Notes: The countries which did not provide answers for any of the survey questions listed here are excluded.

Blank spaces indicate no response. 
Table A.1.6. Barth, Caprio and Levine (2004) survey questions (continued)

Panel C: Legal action by deposit insurance authority and treatment of non-residents

\begin{tabular}{|c|c|c|c|}
\hline Country name & $\begin{array}{l}\text { Can the deposit insurance } \\
\text { agency/fund take legal } \\
\text { action against bank } \\
\text { directors or other bank } \\
\text { officials? } \\
\text { yes }=1 \text { no }=0\end{array}$ & $\begin{array}{c}\text { Has the deposit } \\
\text { insurance agency/fund } \\
\text { ever taken legal action } \\
\text { against bank directors or } \\
\text { other bank officials? } \\
\text { yes }=1 \text { no }=0\end{array}$ & $\begin{array}{l}\text { Are non-residents } \\
\text { treated differently than } \\
\text { residents with respect } \\
\text { to deposit insurance } \\
\text { scheme coverage? } \\
\text { yes }=1 \text { no }=0 \\
\end{array}$ \\
\hline Albania & 1 & 1 & 0 \\
\hline Algeria & 0 & 0 & 0 \\
\hline Argentina & 1 & 1 & 0 \\
\hline Austria & 0 & 0 & 0 \\
\hline Bahrain & 1 & 0 & 0 \\
\hline Belarus & 1 & 0 & 0 \\
\hline Belgium & 1 & 1 & 0 \\
\hline Bolivia & 1 & 1 & 0 \\
\hline Bosnia-Herzegovina & 1 & 0 & 0 \\
\hline Brazil & 0 & 0 & 0 \\
\hline Bulgaria & 0 & 0 & 0 \\
\hline Canada & 1 & 1 & 1 \\
\hline Chile & 0 & 0 & 0 \\
\hline Colombia & 0 & 0 & 0 \\
\hline Croatia & 0 & 0 & 0 \\
\hline Cyprus & 0 & 0 & 0 \\
\hline Czech Rep. & 0 & 0 & 0 \\
\hline Denmark & 0 & 0 & 0 \\
\hline Ecuador & 1 & 1 & 0 \\
\hline El Salvador & 0 & 0 & 0 \\
\hline Estonia & 0 & 0 & 0 \\
\hline Finland & 1 & 0 & 0 \\
\hline France & 1 & 0 & 0 \\
\hline Germany & 1 & 0 & 0 \\
\hline Gibraltar & 0 & 0 & 0 \\
\hline Greece & 0 & 0 & 0 \\
\hline Guatemala & 0 & 0 & 0 \\
\hline Honduras & 1 & 0 & 0 \\
\hline Hungary & 0 & 0 & 0 \\
\hline Iceland & 0 & 0 & 0 \\
\hline India & 0 & 0 & 0 \\
\hline Ireland & 0 & 0 & 0 \\
\hline Isle of Man & 0 & 0 & 0 \\
\hline Italy & 0 & 0 & 0 \\
\hline Japan & 0 & 1 & 0 \\
\hline Jordan & 0 & 0 & 0 \\
\hline Kazakhstan & 1 & 0 & 0 \\
\hline Kenya & 1 & 1 & 0 \\
\hline Korea & 1 & 1 & 0 \\
\hline Latvia & 1 & 0 & 0 \\
\hline Lebanon & 0 & 0 & 0 \\
\hline
\end{tabular}


Table A.1.6 Panel C (continued)

\begin{tabular}{|c|c|c|c|}
\hline \multicolumn{4}{|c|}{ Table A.1.6 Panel C (continued) } \\
\hline Country name & $\begin{array}{c}\text { Can the deposit insurance } \\
\text { agency/fund take legal } \\
\text { action against bank } \\
\text { directors or other bank } \\
\text { officials? } \\
\text { yes }=1 \text { no }=0 \\
\end{array}$ & $\begin{array}{c}\text { Has the deposit } \\
\text { insurance agency/fund } \\
\text { ever taken legal action } \\
\text { against bank directors or } \\
\text { other bank officials? } \\
\text { yes }=1 \text { no=0 }\end{array}$ & $\begin{array}{l}\text { Are non-residents } \\
\text { treated differently than } \\
\text { residents with respect } \\
\text { to deposit insurance } \\
\text { scheme coverage? } \\
\text { yes }=1 \text { no }=0 \\
\end{array}$ \\
\hline Liechtenstein & 1 & 0 & 0 \\
\hline Lithuania & 0 & 0 & 0 \\
\hline Luxembourg & 0 & 0 & 0 \\
\hline Macedonia & 0 & 0 & 0 \\
\hline \multicolumn{4}{|l|}{ Malaysia } \\
\hline Malta & 1 & 0 & 0 \\
\hline Mexico & 1 & & 0 \\
\hline Netherlands & & & 0 \\
\hline Nicaragua & 0 & 0 & 0 \\
\hline Nigeria & 1 & 1 & 0 \\
\hline Norway & 0 & 0 & 0 \\
\hline Oman & 1 & 0 & 0 \\
\hline Paraguay & 1 & 1 & 0 \\
\hline Peru & 1 & 1 & 0 \\
\hline Philippines & 1 & 1 & 0 \\
\hline Poland & 0 & 0 & 0 \\
\hline Portugal & 0 & 0 & 0 \\
\hline Romania & 0 & 0 & 0 \\
\hline Serbia \& Montenegro & 0 & 0 & 0 \\
\hline Slovak Republic & 1 & 1 & 0 \\
\hline Slovenia & 1 & 1 & 0 \\
\hline Spain & 1 & 1 & 0 \\
\hline Sri Lanka & 0 & 0 & 1 \\
\hline Sweden & 0 & 0 & 0 \\
\hline Switzerland & 0 & 0 & 0 \\
\hline Taiwan & 0 & 0 & 0 \\
\hline Thailand & 0 & 0 & 0 \\
\hline Trinidad \& Tobago & 1 & 0 & 0 \\
\hline Turkey & 1 & 1 & 0 \\
\hline Turkmenistan & 0 & 0 & 0 \\
\hline Ukraine & 0 & 0 & 0 \\
\hline United Kingdom & 1 & 0 & 0 \\
\hline United States & 1 & & \\
\hline Venezuela & 1 & 1 & 0 \\
\hline Zimbabwe & 1 & 0 & \\
\hline
\end{tabular}

Notes: The countries which did not provide answers for any of the survey questions listed here are excluded. Blank spaces indicate no response. 
Table A.1.7 Coverage limits and co-insurance over time

\begin{tabular}{|c|c|c|c|c|c|}
\hline Country name & $\begin{array}{c}\text { Country } \\
\text { code }\end{array}$ & year C & erage amount & Currency & $\begin{array}{c}\text { Co- } \\
\text { insurance }\end{array}$ \\
\hline Albania & ALB & 2002 & 700000 & LEK & $15 \%$ \\
\hline Algeria & DZA & 1997 & 600000 & Dinar & $0 \%$ \\
\hline Argentina & ARG & 1979 & 1000000 & pesos & $10 \%$ \\
\hline Argentina & ARG & 1991 & 1000 & & $0 \%$ \\
\hline Argentina & ARG & 1992 & 0 & & $0 \%$ \\
\hline Argentina & ARG & 1995 & 30000 & $\operatorname{Arg} \$$ & $0 \%$ \\
\hline Austria & AUT & 1979 & 200000 & ATS & $0 \%$ \\
\hline Austria & AUT & 1986 & 200000 & ATS & $0 \%$ \\
\hline Austria & AUT & 1995 & 260000 & ATS & $0 \%$ \\
\hline Austria & AUT & 1999 & 20000 & EUR & $10 \%$ \\
\hline Bahamas & BHS & 1999 & 50000 & $\mathrm{~B} \$$ & $0 \%$ \\
\hline Bahrain & BHR & 1993 & 15000 & $\mathrm{BD}$ & $0 \%$ \\
\hline Bangladesh & BGD & 1984 & 100000 & $\mathrm{Tk}$ & $0 \%$ \\
\hline Belarus & BLR & 1996 & 15500 & RBL & $0 \%$ \\
\hline Belarus & BLR & 1997 & 30740 & RBL & $0 \%$ \\
\hline Belarus & BLR & 1998 & 106000 & RBL & $0 \%$ \\
\hline Belarus & BLR & 1999 & 320000 & RBL & $0 \%$ \\
\hline Belarus & BLR & 2000 & 1180000 & RBL & $20 \%$ \\
\hline Belarus & BLR & 2001 & 1580000 & RBL & $20 \%$ \\
\hline Belarus & BLR & 2002 & 1920000 & RBL & $20 \%$ \\
\hline Belarus & BLR & 2003 & 2141000 & RBL & $20 \%$ \\
\hline Belgium & BEL & 1974 & 500000 & Bfr & $0 \%$ \\
\hline Belgium & BEL & 1985 & 500000 & $\mathrm{Bfr}$ & $0 \%$ \\
\hline Belgium & BEL & 1995 & 15000 & ECU & $10 \%$ \\
\hline Belgium & BEL & 1999 & 20000 & EUR & $10 \%$ \\
\hline Bosnia-Herzegovina & $\mathrm{BIH}$ & 1998 & 5000 & KM & $0 \%$ \\
\hline Brazil & BRA & 1995 & 20000 & Reais & $0 \%$ \\
\hline Bulgaria & BGR & 1996 & 2500 & EUR & $0 \%$ \\
\hline Bulgaria & BGR & 1998 & 6900 & BGN & $0 \%$ \\
\hline Bulgaria & BGR & 2001 & 10000 & BGN & $0 \%$ \\
\hline Bulgaria & BGR & 2002 & 15000 & BGN & $0 \%$ \\
\hline Canada & CAN & 1967 & 20000 & Can $\$$ & $0 \%$ \\
\hline Canada & CAN & 1983 & 60000 & Can $\$$ & $0 \%$ \\
\hline Chile & CHL & 1986 & 0 & Chilean Pesos & $10 \%$ \\
\hline Chile & CHL & 1987 & 0 & Chilean Pesos & $10 \%$ \\
\hline Chile & CHL & 1988 & 0 & Chilean Pesos & $10 \%$ \\
\hline Chile & CHL & 1989 & 0 & Chilean Pesos & $10 \%$ \\
\hline Chile & CHL & 1990 & 939119 & Chilean Pesos & $10 \%$ \\
\hline Chile & CHL & 1991 & 1104839 & Chilean Pesos & $10 \%$ \\
\hline Chile & CHL & 1992 & 1256475 & Chilean Pesos & $10 \%$ \\
\hline Chile & CHL & 1993 & 1416424 & Chilean Pesos & $10 \%$ \\
\hline Chile & CHL & 1994 & 1537756 & Chilean Pesos & $10 \%$ \\
\hline Chile & CHL & 1995 & 1664375 & Chilean Pesos & $10 \%$ \\
\hline Chile & CHL & 1996 & 1770724 & Chilean Pesos & $10 \%$ \\
\hline Chile & CHL & 1997 & 1879591 & Chilean Pesos & $10 \%$ \\
\hline Chile & CHL & 1998 & 1958052 & Chilean Pesos & $10 \%$ \\
\hline
\end{tabular}




\begin{tabular}{|c|c|c|c|c|c|}
\hline \multicolumn{5}{|c|}{ Table A.1.7 (continued) } & \multirow[b]{2}{*}{$\begin{array}{c}\text { Co- } \\
\text { insurance }\end{array}$} \\
\hline Country name & $\begin{array}{c}\text { Countr } \\
\text { code }\end{array}$ & & erage amount & Currency & \\
\hline Chile & CHL & 1999 & 2008928 & Chilean Pesos & $10 \%$ \\
\hline Chile & CHL & 2000 & 2102656 & Chilean Pesos & $10 \%$ \\
\hline Chile & CHL & 2001 & 2168355 & Chilean Pesos & $10 \%$ \\
\hline Chile & $\mathrm{CHL}$ & 2002 & 2232549 & Chilean Pesos & $10 \%$ \\
\hline Chile & $\mathrm{CHL}$ & 2003 & 2256000 & Chilean Pesos & $10 \%$ \\
\hline Colombia & $\mathrm{COL}$ & 1985 & 10000000 & Pesos & $25 \%$ \\
\hline Colombia & $\mathrm{COL}$ & 2001 & 20000000 & Pesos & $25 \%$ \\
\hline Croatia & HRV & 1997 & 100000 & & $0 \%$ \\
\hline Cyprus & CYP & 2000 & 20000 & EUR & $10 \%$ \\
\hline Czech Rep. & CZE & 1994 & 100000 & CZK & $20 \%$ \\
\hline Czech Rep. & CZE & 1996 & 4000000 & $\mathrm{CZK}$ & $0 \%$ \\
\hline Czech Rep. & CZE & 1998 & 400000 & $\mathrm{CZK}$ & $10 \%$ \\
\hline Czech Rep. & CZE & 2001 & 530000 & $\mathrm{CZK}$ & $10 \%$ \\
\hline Czech Rep. & CZE & 2002 & 25000 & EUR & $10 \%$ \\
\hline Denmark & DNK & 1987 & 250000 & DKK & $0 \%$ \\
\hline Denmark & DNK & 1995 & 300000 & DKK & $0 \%$ \\
\hline Dominican Republic & DOM & 1998 & 8000 & RD\$ & $0 \%$ \\
\hline Dominican Republic & DOM & 2002 & Full & RD\$ & $0 \%$ \\
\hline Ecuador & ECU & 1999 & Full & & $0 \%$ \\
\hline Ecuador & ECU & 2001 & er capita GDP & & $0 \%$ \\
\hline El Salvador & SLV & 1999 & 35000 & Colon & $0 \%$ \\
\hline El Salvador & SLV & 2000 & 55000 & Colon & $0 \%$ \\
\hline El Salvador & SLV & 2002 & 58424 & Colon & $0 \%$ \\
\hline Estonia & EST & 1998 & 20000 & Estonian kroons & $10 \%$ \\
\hline Estonia & EST & 2000 & 40000 & Estonian kroons & $10 \%$ \\
\hline Estonia & EST & 2002 & 40000 & Estonian kroons & $10 \%$ \\
\hline Estonia & EST & 2003 & 100000 & Estonian kroons & $10 \%$ \\
\hline Finland & FIN & 1969 & Full & & $0 \%$ \\
\hline Finland & FIN & 1993 & Full & & \\
\hline Finland & FIN & 1998 & 150000 & Marka & $0 \%$ \\
\hline Finland & FIN & 1999 & 25000 & EUR & $0 \%$ \\
\hline France & FRA & 1980 & 200000 & FFR & $0 \%$ \\
\hline France & FRA & 1986 & 400000 & FFR & $0 \%$ \\
\hline France & FRA & 1999 & 70000 & EUR & $0 \%$ \\
\hline Germany & DEU & 1966 & 20000 & DM & $10 \%$ \\
\hline Germany & DEU & 1998 & 20000 & EUR & $10 \%$ \\
\hline Gibraltar & GIB & 1997 & 20000 & Sterling & $10 \%$ \\
\hline Greece & GRC & 1995 & 20000 & EUR / ECU & $0 \%$ \\
\hline Guatemala & GTM & 1999 & 20000 & Quetzales & $0 \%$ \\
\hline Honduras & HND & 1999 & Full & & $0 \%$ \\
\hline Honduras & HND & 2003 & 165000 & & $0 \%$ \\
\hline Hungary & HUN & 1993 & 1000000 & HUF & $0 \%$ \\
\hline Hungary & HUN & 2003 & 3222222 & HUF & $0 \%$ \\
\hline Iceland & ISL & 1998 & 1700000 & ISK & $0 \%$ \\
\hline Iceland & ISL & 1999 & 1700000 & ISK & $0 \%$ \\
\hline Iceland & ISL & 2000 & 1836000 & ISK & $0 \%$ \\
\hline Iceland & ISL & 2001 & 2108000 & ISK & $0 \%$ \\
\hline
\end{tabular}


Table A.1.7 (continued)

\begin{tabular}{|c|c|c|c|c|c|}
\hline Country name & $\begin{array}{c}\text { Countr } \\
\text { code }\end{array}$ & yYearC & verage amount & Currency & $\begin{array}{c}\text { Co- } \\
\text { insurance }\end{array}$ \\
\hline Iceland & ISL & 2002 & 1972000 & ISK & $0 \%$ \\
\hline Iceland & ISL & 2003 & 2091000 & ISK & $0 \%$ \\
\hline India & IND & 1961 & 1500 & Rs & $0 \%$ \\
\hline India & IND & 1968 & 5000 & Rs & $0 \%$ \\
\hline India & IND & 1970 & 10000 & Rs & $0 \%$ \\
\hline India & IND & 1976 & 20000 & Rs & $0 \%$ \\
\hline India & IND & 1980 & 30000 & Rs & $0 \%$ \\
\hline India & IND & 1993 & 100000 & Rs & $0 \%$ \\
\hline Indonesia & IDN & 1998 & Full & & \\
\hline Ireland & IRL & 1989 & 15000 & Pounds & $50 \%$ \\
\hline Ireland & IRL & 1995 & 15000 & EUR & $10 \%$ \\
\hline Ireland & IRL & 1999 & 20000 & EUR & $10 \%$ \\
\hline Isle of Man & IMY & 1991 & 20000 & Sterling pounds & $25 \%$ \\
\hline Italy & ITA & 1986 & 200000000 & Italian lire & $0 \%$ \\
\hline Jamaica & JAM & 1998 & 200000 & $\mathrm{~J} \$$ & $0 \%$ \\
\hline Jamaica & JAM & 2001 & 300000 & $\mathrm{~J} \$$ & $0 \%$ \\
\hline Japan & JPN & 1971 & 1000000 & Yens & $0 \%$ \\
\hline Japan & JPN & 1974 & 3000000 & Yens & $0 \%$ \\
\hline Japan & JPN & 1986 & 10000000 & Yens & $0 \%$ \\
\hline Japan & JPN & 1996 & Full & Yens & $0 \%$ \\
\hline Japan & JPN & 2002 & Full & & $0 \%$ \\
\hline Japan & JPN & 2002 & 10000000 & Yens & $0 \%$ \\
\hline Jordan & JOR & 2000 & 10000 & JD & $0 \%$ \\
\hline Kazakhstan & KAZ & 1999 & 200000 & Tenge & $0 \%$ \\
\hline Kazakhstan & KAZ & 2003 & 400000 & Tenge & $0 \%$ \\
\hline Kenya & KEN & 1988 & 50000 & K Sh & $0 \%$ \\
\hline Kenya & KEN & 2000 & 100000 & K Sh & $0 \%$ \\
\hline Korea & KOR & 1996 & 20000000 & Won & $0 \%$ \\
\hline Korea & KOR & 1997 & Full & Won & $0 \%$ \\
\hline Korea & KOR & 1998 & Full & Won & $0 \%$ \\
\hline Korea & KOR & 2001 & 50000000 & Won & $0 \%$ \\
\hline Kuwait & KUW & 1982 & Full & & \\
\hline Latvia & LVA & 1998 & 500 & LVL & $0 \%$ \\
\hline Latvia & LVA & 2000 & 1000 & LVL & $0 \%$ \\
\hline Latvia & LVA & 2001 & 3000 & LVL & $0 \%$ \\
\hline Lebanon & LBN & 1967 & 30000 & LL & $0 \%$ \\
\hline Lebanon & LBN & 1986 & 250000 & LL & $0 \%$ \\
\hline Lebanon & LBN & 1988 & 1000000 & LL & $0 \%$ \\
\hline Lebanon & LBN & 1991 & 5000000 & LL & $0 \%$ \\
\hline Liechtenstein & LIE & 1992 & 20000 & EUR & $0 \%$ \\
\hline Lithuania & LTU & 1996 & 45000 & LTL & $10 \%$ \\
\hline Luxembourg & LUX & 1989 & 500000 & LUF & $10 \%$ \\
\hline Luxembourg & LUX & 2000 & 20000 & EUR & $10 \%$ \\
\hline Macedonia & MKD & 1996 & 10000 & $\mathrm{DM}$ & $25 \%$ \\
\hline Macedonia & MKD & 2000 & 7500 & EUR & $10 \%$ \\
\hline Macedonia & MKD & 2002 & 20000 & EUR & $10 \%$ \\
\hline Malaysia & MAL & 1998 & Full & & \\
\hline
\end{tabular}




\begin{tabular}{|c|c|c|c|c|c|}
\hline \multicolumn{6}{|c|}{ Table A.1.7 (continued) } \\
\hline \multirow{2}{*}{$\begin{array}{l}\text { Country name } \\
\text { Malta }\end{array}$} & \multicolumn{3}{|c|}{$\begin{array}{l}\text { Country Year Coverage amount } \\
\text { code }\end{array}$} & \multirow{2}{*}{$\begin{array}{c}\text { Currency } \\
\text { LM }\end{array}$} & \multirow{2}{*}{$\begin{array}{c}\begin{array}{c}\text { Co- } \\
\text { insurance }\end{array} \\
0 \%\end{array}$} \\
\hline & MLT & 2003 & 8600 & & \\
\hline Marshall Islands & MHL & 1975 & 40000 & USD & $0 \%$ \\
\hline Marshall Islands & MHL & 1980 & 100000 & USD & $0 \%$ \\
\hline Mexico & MEX & 1990 & Full & & $0 \%$ \\
\hline Mexico & MEX & 1998 & Unlimited & & $0 \%$ \\
\hline Mexico & MEX & 2003 & 32262340 & Mexican Pesos & $0 \%$ \\
\hline Mexico & MEX & 2004 & 16762350 & Mexican Pesos & $0 \%$ \\
\hline Mexico & MEX & 2005 & 1308000 & Mexican Pesos & \\
\hline Moldova & MLD & 2004 & 4500 & MDL & $0 \%$ \\
\hline Netherlands & NLD & 1979 & 25000 & HFL & $0 \%$ \\
\hline Netherlands & NLD & 1996 & 44000 & HFL & $0 \%$ \\
\hline Netherlands & NLD & 1998 & 20000 & EUR & $0 \%$ \\
\hline Nicaragua & NIC & 2001 & 20000 & USD & $0 \%$ \\
\hline Nigeria & NGA & 1988 & 50000 & $\mathrm{~N}$ & $0 \%$ \\
\hline Norway & NOR & 1961 & Full & & $0 \%$ \\
\hline Norway & NOR & 1997 & 2000000 & NOK & $0 \%$ \\
\hline Oman & $\mathrm{OMN}$ & 1995 & 20000 & RO & $25 \%$ \\
\hline Paraguay & PRY & 2003 & 64207500 & & $0 \%$ \\
\hline Peru & PER & 1991 & 2500 & $\mathrm{~S}$ & $0 \%$ \\
\hline Peru & PER & 1992 & 4307 & S & $0 \%$ \\
\hline Peru & PER & 1993 & 9000 & $\mathrm{~S}$ & $0 \%$ \\
\hline Peru & PER & 1994 & 10151 & $\mathrm{~S}$ & $0 \%$ \\
\hline Peru & PER & 1995 & 10948 & S & $0 \%$ \\
\hline Peru & PER & 1996 & 12061 & $\mathrm{~S}$ & $0 \%$ \\
\hline Peru & PER & 1997 & 12814 & $\mathrm{~S}$ & $0 \%$ \\
\hline Peru & PER & 1998 & 62000 & $\mathrm{~S}$ & $0 \%$ \\
\hline Peru & PER & 1999 & 65163 & $\mathrm{~S}$ & $0 \%$ \\
\hline Peru & PER & 2000 & 67874 & $\mathrm{~S}$ & $0 \%$ \\
\hline Peru & PER & 2001 & 66782 & S & $0 \%$ \\
\hline Peru & PER & 2002 & 67855 & S & $0 \%$ \\
\hline Peru & PER & 2003 & 68474 & $\mathrm{~S}$ & $0 \%$ \\
\hline Philippines & PHL & 1963 & 10000 & $\mathrm{P}$ & $0 \%$ \\
\hline Philippines & PHL & 1978 & 15000 & $\mathrm{P}$ & $0 \%$ \\
\hline Philippines & PHL & 1984 & 40000 & $\mathrm{P}$ & $0 \%$ \\
\hline Philippines & PHL & 1992 & 100000 & $\mathrm{P}$ & $0 \%$ \\
\hline Poland & POL & 1995 & 3000 & EUR & $10 \%$ \\
\hline Poland & POL & 1997 & 4000 & EUR & $10 \%$ \\
\hline Poland & POL & 1998 & 5000 & EUR & $10 \%$ \\
\hline Poland & POL & 1999 & 8000 & EUR & $10 \%$ \\
\hline Poland & POL & 2000 & 11000 & EUR & $10 \%$ \\
\hline Poland & POL & 2001 & 15000 & EUR & $10 \%$ \\
\hline Poland & POL & 2002 & 18000 & EUR & $10 \%$ \\
\hline Poland & POL & 2003 & 22500 & EUR & $10 \%$ \\
\hline Portugal & PRT & 1992 & 45000 & ECU & $50 \%$ \\
\hline Portugal & PRT & 1999 & 25000 & EUR & $0 \%$ \\
\hline Romania & ROM & 1996 & 10000000 & ROL & $0 \%$ \\
\hline Romania & ROM & 1997 & 20120000 & ROL & $0 \%$ \\
\hline
\end{tabular}




\begin{tabular}{|c|c|c|c|c|c|}
\hline \multicolumn{6}{|c|}{ Table A.1.7 (continued) } \\
\hline Country name & $\begin{array}{c}\text { Country } \\
\text { code }\end{array}$ & y Year & $\begin{array}{c}\text { Coverage } \\
\text { amount }\end{array}$ & Currency & $\begin{array}{c}\text { Co- } \\
\text { insurance }\end{array}$ \\
\hline Romania & ROM & 1998 & 31211000 & ROL & $0 \%$ \\
\hline Romania & ROM & 1999 & 46253000 & ROL & $0 \%$ \\
\hline Romania & ROM & 2000 & 65169000 & ROL & $0 \%$ \\
\hline Romania & ROM & 2001 & 88505000 & ROL & $0 \%$ \\
\hline Romania & $\mathrm{ROM}$ & 2002 & 109795000 & ROL & $0 \%$ \\
\hline Romania & ROM & 2003 & 125222000 & ROL & $0 \%$ \\
\hline Russia & RUS & 2003 & 100000 & Rubles & $50 \%$ \\
\hline Serbia \& Montenegro & YUG & 2001 & 5000 & Dinar & $0 \%$ \\
\hline Slovak Republic & SVK & 1996 & 215850 & SKK & $0 \%$ \\
\hline Slovak Republic & SVK & 1997 & 244620 & SKK & $0 \%$ \\
\hline Slovak Republic & SVK & 1998 & 276780 & SKK & $0 \%$ \\
\hline Slovak Republic & SVK & 1999 & 300090 & SKK & $0 \%$ \\
\hline Slovak Republic & SVK & 2000 & 321840 & SKK & $0 \%$ \\
\hline Slovak Republic & SVK & 2001 & 381000 & SKK & $0 \%$ \\
\hline Slovak Republic & SVK & 2002 & 549555.56 & SKK & $0 \%$ \\
\hline Slovak Republic & SVK & 2003 & 22222.22 & EUR & $0 \%$ \\
\hline Slovenia & SVN & 1991 & Full & & \\
\hline Slovenia & SVN & 2001 & 4200000 & SIT & $0 \%$ \\
\hline Slovenia & SVN & 2003 & 5100000 & SIT & $0 \%$ \\
\hline Spain & ESP & 1977 & 500000 & Pesetas & $0 \%$ \\
\hline Spain & ESP & 1980 & 750000 & Pesetas & $0 \%$ \\
\hline Spain & ESP & 1981 & 1500000 & Pesetas & $0 \%$ \\
\hline Spain & ESP & 1995 & 15000 & ECU & $0 \%$ \\
\hline Spain & ESP & 1996 & 15000 & EUR & $0 \%$ \\
\hline Spain & ESP & 2000 & 20000 & EUR & $0 \%$ \\
\hline Sri Lanka & LKA & 1987 & 100000 & Rs & $0 \%$ \\
\hline Sweden & SWE & 1992 & Full & & \\
\hline Sweden & SWE & 1996 & 250000 & SEK & $0 \%$ \\
\hline Switzerland & CHE & 1934 & 5000 & $\mathrm{CHF}$ & $0 \%$ \\
\hline Switzerland & CHE & 1971 & 10000 & $\mathrm{CHF}$ & $0 \%$ \\
\hline Switzerland & CHE & 1984 & 30000 & $\mathrm{CHF}$ & $0 \%$ \\
\hline Switzerland & CHE & 1993 & 30000 & & $0 \%$ \\
\hline Switzerland & CHE & 1997 & 30000 & $\mathrm{CHF}$ & $0 \%$ \\
\hline Taiwan & TWN & 1985 & 700000 & NT\$ & $0 \%$ \\
\hline Taiwan & TWN & 1987 & 1000000 & NT\$ & $0 \%$ \\
\hline Tanzania & TZA & 1994 & 250000 & TZS & $0 \%$ \\
\hline Thailand & THA & 1997 & Full & & $0 \%$ \\
\hline Trinidad \& Tobago & TTO & 1986 & 50000 & TT \$ & $0 \%$ \\
\hline Trinidad \& Tobago & TTO & 1998 & 50000 & TT \$ & $0 \%$ \\
\hline Turkey & TUR & 1983 & 3000000 & $\mathrm{TL}$ & $0 \%$ \\
\hline Turkey & TUR & 1986 & 6000000 & $\mathrm{TL}$ & $0 \%$ \\
\hline Turkey & TUR & 1992 & 50000000 & $\mathrm{TL}$ & $0 \%$ \\
\hline Turkey & TUR & 1994 & 150000000 & $\mathrm{TL}$ & $0 \%$ \\
\hline Turkey & TUR & 1995 & Full & $\mathrm{TL}$ & $0 \%$ \\
\hline Turkey & TUR & 2000 & Full & $\mathrm{TL}$ & $0 \%$ \\
\hline Turkey & TUR & 2002 & 50000000000 & $\mathrm{TL}$ & $0 \%$ \\
\hline Turkey & TUR & 2003 & Full & TL & $0 \%$ \\
\hline
\end{tabular}




\begin{tabular}{|c|c|c|c|c|c|}
\hline \multicolumn{6}{|c|}{ Table A.1.7 (continued) } \\
\hline Country name & $\begin{array}{c}\text { Country } \\
\text { code }\end{array}$ & y Year & $\begin{array}{c}\text { Coverage } \\
\text { amount }\end{array}$ & Currency & $\begin{array}{c}\text { Co- } \\
\text { insurance }\end{array}$ \\
\hline Turkmenistan & & 2000 & Full & & $0 \%$ \\
\hline Uganda & UGA & 1994 & 3000000 & Shs & $0 \%$ \\
\hline Ukraine & UKR & 1998 & 1200 & UAH & $0 \%$ \\
\hline Ukraine & UKR & 2003 & 1500 & UAH & $0 \%$ \\
\hline Ukraine & UKR & 2004 & 2000 & UAH & $0 \%$ \\
\hline United Kingdom & GBR & 1982 & 10000 & Sterling pounds & $25 \%$ \\
\hline United Kingdom & GBR & 1987 & 20000 & Sterling pounds & $25 \%$ \\
\hline United Kingdom & GBR & 1995 & 20000 & Sterling pounds & $10 \%$ \\
\hline United Kingdom & GBR & 2001 & 35000 & Sterling pounds & $10 \%$ \\
\hline United States & USA & 1934 & 5000 & USD & $0 \%$ \\
\hline United States & USA & 1950 & 10000 & USD & $0 \%$ \\
\hline United States & USA & 1966 & 15000 & USD & $0 \%$ \\
\hline United States & USA & 1969 & 20000 & USD & $0 \%$ \\
\hline United States & USA & 1974 & 40000 & USD & $0 \%$ \\
\hline United States & USA & 1980 & 100000 & USD & $0 \%$ \\
\hline Venezuela & VEN & 1985 & 250000 & $\mathrm{Bs}$ & $0 \%$ \\
\hline Venezuela & VEN & 1994 & 1000000 & Bs & $0 \%$ \\
\hline Venezuela & VEN & 1995 & 4000000 & Bs & $0 \%$ \\
\hline Venezuela & VEN & 2002 & 10000000 & Bs & $0 \%$ \\
\hline Vietnam & VNM & 2000 & 30000000 & VND & $0 \%$ \\
\hline Zimbabwe & ZWE & 2003 & 200000 & Zimbabwe \$ & $0 \%$ \\
\hline
\end{tabular}


Table A.1.8 Coverage ratios 1999-2003

\begin{tabular}{|c|c|c|c|}
\hline Country name & Year & $\begin{array}{c}\text { Coverage to GDP per } \\
\text { capita ratio }\end{array}$ & $\begin{array}{c}\text { Coverage to deposits per } \\
\text { capita ratio }\end{array}$ \\
\hline Albania & 2002 & 3.30 & \\
\hline Albania & 2003 & 2.97 & \\
\hline Algeria & 1999 & 5.67 & 19.33 \\
\hline Algeria & 2000 & 4.53 & 16.89 \\
\hline Algeria & 2001 & 4.37 & 13.29 \\
\hline Algeria & 2002 & 4.22 & \\
\hline Algeria & 2003 & 3.74 & 8.03 \\
\hline Argentina & 1999 & 3.87 & 14.56 \\
\hline Argentina & 2000 & 3.91 & 14.25 \\
\hline Argentina & 2001 & 4.19 & 17.63 \\
\hline Argentina & 2002 & 3.64 & 16.04 \\
\hline Argentina & 2003 & 3.06 & 13.31 \\
\hline Austria & 1999 & 0.82 & 0.98 \\
\hline Austria & 2000 & 0.78 & 0.97 \\
\hline Austria & 2001 & 0.77 & 0.90 \\
\hline Austria & 2002 & 0.75 & 0.90 \\
\hline Austria & 2003 & 0.72 & 0.84 \\
\hline Bahamas & 1999 & 3.31 & 4.98 \\
\hline Bahamas & 2000 & 3.17 & 4.62 \\
\hline Bahamas & 2001 & 3.13 & 4.45 \\
\hline Bahamas & 2002 & 3.11 & 4.39 \\
\hline Bahamas & 2003 & 3.01 & 4.27 \\
\hline Bahrain & 1999 & 3.89 & 5.38 \\
\hline Bahrain & 2000 & 3.40 & 4.84 \\
\hline Bahrain & 2001 & 3.46 & 4.46 \\
\hline Bahrain & 2002 & 3.49 & 4.36 \\
\hline Bangladesh & 1999 & 5.86 & 21.64 \\
\hline Bangladesh & 2000 & 5.53 & 18.56 \\
\hline Bangladesh & 2001 & 5.26 & 16.30 \\
\hline Bangladesh & 2002 & 4.97 & 14.55 \\
\hline Bangladesh & 2003 & 4.59 & 13.03 \\
\hline Belarus & 1999 & 1.06 & 7.75 \\
\hline Belarus & 2000 & 1.29 & 8.68 \\
\hline Belarus & 2001 & 0.92 & 7.68 \\
\hline Belarus & 2002 & 0.75 & 5.81 \\
\hline Belarus & 2003 & 0.59 & 4.04 \\
\hline Belgium & 1999 & 0.87 & 0.98 \\
\hline Belgium & 2000 & 0.83 & 0.99 \\
\hline Belgium & 2001 & 0.81 & 0.92 \\
\hline Belgium & 2002 & 0.79 & 0.88 \\
\hline Belgium & 2003 & 0.77 & 0.81 \\
\hline Bosnia-Herzegovina & 1999 & 2.25 & \\
\hline Bosnia-Herzegovina & 2000 & 2.07 & \\
\hline Bosnia-Herzegovina & 2001 & 1.85 & \\
\hline Bosnia-Herzegovina & 2002 & 1.77 & \\
\hline Bosnia-Herzegovina & 2003 & 1.70 & \\
\hline
\end{tabular}




\begin{tabular}{|c|c|c|c|}
\hline \multicolumn{4}{|c|}{ Table A.1.8 (continued) } \\
\hline Country name & Year & $\begin{array}{c}\text { Coverage to GDP per } \\
\text { capita ratio }\end{array}$ & $\begin{array}{c}\text { Coverage to deposits per } \\
\text { capita ratio }\end{array}$ \\
\hline Brazil & 1999 & 3.50 & 12.22 \\
\hline Brazil & 2000 & 3.09 & 11.93 \\
\hline Brazil & 2001 & 2.87 & 10.78 \\
\hline Brazil & 2002 & 2.64 & 8.90 \\
\hline Brazil & 2003 & 2.33 & 8.70 \\
\hline Bulgaria & 1999 & 2.38 & 11.33 \\
\hline Bulgaria & 2000 & 2.10 & 9.49 \\
\hline Bulgaria & 2001 & 2.66 & 9.85 \\
\hline Bulgaria & 2002 & 2.43 & 8.52 \\
\hline Bulgaria & 2003 & 3.41 & 10.87 \\
\hline Canada & 1999 & 1.90 & 3.31 \\
\hline Canada & 2000 & 1.76 & 2.90 \\
\hline Canada & 2001 & 1.73 & 2.74 \\
\hline Canada & 2002 & 1.68 & 2.63 \\
\hline Canada & 2003 & 1.62 & 2.47 \\
\hline Chile & 1999 & 0.81 & 1.98 \\
\hline Chile & 2000 & 0.79 & 1.97 \\
\hline Chile & 2001 & 0.79 & 2.02 \\
\hline Chile & 2002 & 0.79 & 2.12 \\
\hline Chile & 2003 & 0.71 & 2.01 \\
\hline Colombia & 1999 & 2.74 & 12.93 \\
\hline Colombia & 2000 & 2.42 & 11.49 \\
\hline Colombia & 2001 & 4.58 & 19.95 \\
\hline Colombia & 2002 & 4.32 & 17.95 \\
\hline Colombia & 2003 & 3.98 & 16.82 \\
\hline Croatia & 1999 & 3.09 & 8.70 \\
\hline Croatia & 2000 & 2.87 & 6.65 \\
\hline Croatia & 2001 & 2.69 & 4.51 \\
\hline Croatia & 2002 & 2.48 & 4.13 \\
\hline Croatia & 2003 & 2.35 & 3.78 \\
\hline Cyprus & 2000 & 2.76 & 2.42 \\
\hline Cyprus & 2001 & 2.59 & 2.14 \\
\hline Cyprus & 2002 & 2.48 & 1.97 \\
\hline Cyprus & 2003 & 2.30 & 1.92 \\
\hline Czech Rep. & 1999 & 2.16 & 3.78 \\
\hline Czech Rep. & 2000 & 2.07 & 3.24 \\
\hline Czech Rep. & 2001 & 2.49 & 3.78 \\
\hline Czech Rep. & 2002 & 3.55 & 5.30 \\
\hline Czech Rep. & 2003 & 3.43 & 5.10 \\
\hline Denmark & 1999 & 1.32 & 2.51 \\
\hline Denmark & 2000 & 1.25 & 2.65 \\
\hline Denmark & 2001 & 1.21 & 2.57 \\
\hline Denmark & 2002 & 1.18 & 2.45 \\
\hline Denmark & 2003 & 1.15 & 2.32 \\
\hline Dominican Republic & 1999 & 0.24 & 0.90 \\
\hline Dominican Republic & 2000 & 0.21 & 0.74 \\
\hline Dominican Republic & 2001 & 0.19 & 0.58 \\
\hline El Salvador & 1999 & 1.97 & 39.81 \\
\hline
\end{tabular}




\begin{tabular}{|c|c|c|c|}
\hline \multicolumn{4}{|c|}{ Table A.1.8 (continued) } \\
\hline Country name & Year & $\begin{array}{c}\text { Coverage to GDP per } \\
\text { capita ratio }\end{array}$ & $\begin{array}{c}\text { Coverage to deposits per } \\
\text { capita ratio }\end{array}$ \\
\hline El Salvador & 2000 & 3.00 & 61.53 \\
\hline El Salvador & 2001 & 2.91 & 58.09 \\
\hline El Salvador & 2002 & 3.05 & 63.31 \\
\hline El Salvador & 2003 & 3.03 & 62.57 \\
\hline Estonia & 1999 & 0.36 & 1.34 \\
\hline Estonia & 2000 & 0.63 & 2.05 \\
\hline Estonia & 2001 & 0.56 & 1.61 \\
\hline Estonia & 2002 & 0.50 & 1.42 \\
\hline Estonia & 2003 & 1.16 & 3.13 \\
\hline Finland & 1999 & 1.08 & 2.13 \\
\hline Finland & 2000 & 0.99 & 2.12 \\
\hline Finland & 2001 & 0.96 & 2.03 \\
\hline Finland & 2002 & 0.93 & 1.91 \\
\hline Finland & 2003 & 0.91 & 1.85 \\
\hline France & 1999 & 3.03 & 4.69 \\
\hline France & 2000 & 2.90 & 4.57 \\
\hline France & 2001 & 2.81 & 4.29 \\
\hline France & 2002 & 2.74 & 4.23 \\
\hline France & 2003 & 2.70 & 3.87 \\
\hline Germany & 1999 & 0.83 & 0.88 \\
\hline Germany & 2000 & 0.81 & 0.88 \\
\hline Germany & 2001 & 0.80 & 0.83 \\
\hline Germany & 2002 & 0.78 & 0.81 \\
\hline Germany & 2003 & 0.78 & 0.79 \\
\hline Greece & 1999 & 1.87 & 3.21 \\
\hline Greece & 2000 & 1.74 & 3.07 \\
\hline Greece & 2001 & 1.62 & 1.66 \\
\hline Greece & 2002 & 1.51 & 1.68 \\
\hline Greece & 2003 & 1.39 & 1.67 \\
\hline Guatemala & 1999 & 1.64 & 10.42 \\
\hline Guatemala & 2000 & 1.52 & 8.20 \\
\hline Guatemala & 2001 & 1.42 & 7.05 \\
\hline Guatemala & 2002 & 1.32 & 6.33 \\
\hline Guatemala & 2003 & 1.25 & 5.66 \\
\hline Honduras & 2003 & 9.48 & 22.16 \\
\hline Hungary & 1999 & 0.88 & 2.27 \\
\hline Hungary & 2000 & 0.77 & 1.98 \\
\hline Hungary & 2001 & 0.69 & 1.72 \\
\hline Hungary & 2002 & 0.60 & 1.49 \\
\hline Hungary & 2003 & 1.63 & 4.02 \\
\hline Iceland & 1999 & 0.78 & 1.80 \\
\hline Iceland & 2000 & 0.78 & 1.77 \\
\hline Iceland & 2001 & 0.80 & 1.77 \\
\hline Iceland & 2002 & 0.72 & 1.51 \\
\hline India & 1999 & 5.16 & 12.18 \\
\hline India & 2000 & 4.86 & 10.59 \\
\hline India & 2001 & 4.52 & 9.36 \\
\hline India & 2002 & 4.24 & 8.08 \\
\hline
\end{tabular}




\begin{tabular}{|c|c|c|c|}
\hline \multicolumn{4}{|c|}{ Table A.1.8 (continued) } \\
\hline Country name & Year & $\begin{array}{c}\text { Coverage to GDP per } \\
\text { capita ratio }\end{array}$ & $\begin{array}{c}\text { Coverage to deposits per } \\
\text { capita ratio }\end{array}$ \\
\hline India & 2003 & 3.87 & 7.34 \\
\hline Ireland & 1999 & 0.84 & 1.04 \\
\hline Ireland & 2000 & 0.74 & 0.92 \\
\hline Ireland & 2001 & 0.67 & 0.85 \\
\hline Ireland & 2002 & 0.60 & 0.78 \\
\hline Ireland & 2003 & 0.60 & 0.71 \\
\hline Italy & 1999 & 5.37 & 10.33 \\
\hline Italy & 2000 & 5.11 & 9.97 \\
\hline Italy & 2001 & 4.91 & 9.39 \\
\hline Italy & 2002 & 4.75 & 8.68 \\
\hline Italy & 2003 & 4.58 & 8.59 \\
\hline Jamaica & 1999 & 1.82 & 4.52 \\
\hline Jamaica & 2000 & 1.63 & 3.93 \\
\hline Jamaica & 2001 & 2.23 & 5.47 \\
\hline Jamaica & 2002 & 2.06 & 4.90 \\
\hline Jamaica & 2003 & 1.74 & 4.50 \\
\hline Japan & 2002 & 2.54 & 2.14 \\
\hline Japan & 2003 & 2.54 & 2.11 \\
\hline Jordan & 2000 & 8.14 & 9.00 \\
\hline Jordan & 2001 & 8.04 & 8.60 \\
\hline Jordan & 2002 & 7.84 & 8.02 \\
\hline Jordan & 2003 & 7.59 & 7.22 \\
\hline Kazakhstan & 1999 & 1.52 & 18.21 \\
\hline Kazakhstan & 2000 & 1.16 & 10.56 \\
\hline Kazakhstan & 2001 & 0.92 & 7.04 \\
\hline Kazakhstan & 2002 & 0.78 & 5.28 \\
\hline Kazakhstan & 2003 & 1.34 & 8.58 \\
\hline Kenya & 1999 & 1.98 & 5.33 \\
\hline Kenya & 2000 & 3.78 & 10.42 \\
\hline Kenya & 2001 & 3.48 & 10.20 \\
\hline Kenya & 2002 & 3.23 & 9.48 \\
\hline Kenya & 2003 & 3.07 & 8.51 \\
\hline Korea & 2001 & 4.27 & 5.27 \\
\hline Korea & 2002 & 4.00 & 4.77 \\
\hline Korea & 2003 & 3.32 & 4.49 \\
\hline Latvia & 1999 & 0.31 & 1.82 \\
\hline Latvia & 2000 & 0.55 & 2.67 \\
\hline Latvia & 2001 & 1.47 & 6.49 \\
\hline Latvia & 2002 & 1.35 & 5.19 \\
\hline Lebanon & 1999 & 0.86 & 0.49 \\
\hline Lebanon & 2000 & 0.87 & 0.45 \\
\hline Lebanon & 2001 & 0.87 & 0.43 \\
\hline Lebanon & 2002 & 0.85 & 0.40 \\
\hline Lebanon & 2003 & 0.79 & 0.37 \\
\hline Lithuania & 1999 & 3.73 & 25.55 \\
\hline Lithuania & 2000 & 3.53 & 20.25 \\
\hline Lithuania & 2001 & 3.30 & 16.07 \\
\hline Lithuania & 2002 & 3.09 & 14.09 \\
\hline
\end{tabular}




\begin{tabular}{|c|c|c|c|}
\hline \multicolumn{4}{|c|}{ Table A.1.8 (continued) } \\
\hline Country name & Year & $\begin{array}{c}\text { Coverage to GDP per } \\
\text { capita ratio }\end{array}$ & $\begin{array}{c}\text { Coverage to deposits per } \\
\text { capita ratio }\end{array}$ \\
\hline Lithuania & 2003 & 2.79 & 12.05 \\
\hline Luxembourg & 1999 & 0.34 & 0.11 \\
\hline Luxembourg & 2000 & 0.41 & 0.13 \\
\hline Luxembourg & 2001 & 0.40 & 0.12 \\
\hline Luxembourg & 2002 & 0.40 & 0.13 \\
\hline Luxembourg & 2003 & 0.39 & 0.12 \\
\hline Macedonia & 1999 & 3.75 & 27.66 \\
\hline Macedonia & 2000 & 4.47 & 30.03 \\
\hline Macedonia & 2001 & 4.73 & 18.33 \\
\hline Macedonia & 2002 & 10.27 & 46.04 \\
\hline Macedonia & 2003 & 9.92 & 38.82 \\
\hline Marshall Islands & 1999 & 52.49 & \\
\hline Marshall Islands & 2000 & 53.10 & \\
\hline Marshall Islands & 2001 & 51.62 & \\
\hline Marshall Islands & 2002 & 50.30 & \\
\hline Marshall Islands & 2003 & 49.96 & \\
\hline Mexico & 2003 & 489.14 & 1955.03 \\
\hline Micronesia & 1999 & 55.26 & 105.45 \\
\hline Micronesia & 2000 & 52.94 & 108.35 \\
\hline Micronesia & 2001 & 52.25 & 104.55 \\
\hline Micronesia & 2002 & 52.73 & 121.17 \\
\hline Micronesia & 2003 & 51.83 & 127.94 \\
\hline Netherlands & 1999 & 0.85 & 0.92 \\
\hline Netherlands & 2000 & 0.79 & 0.82 \\
\hline Netherlands & 2001 & 0.75 & 0.75 \\
\hline Netherlands & 2002 & 0.73 & 0.73 \\
\hline Netherlands & 2003 & 0.72 & 0.68 \\
\hline Nicaragua & 2001 & 26.85 & 78.30 \\
\hline Nicaragua & 2002 & 27.44 & 74.89 \\
\hline Nicaragua & 2003 & 27.52 & 72.83 \\
\hline Nigeria & 1999 & 1.84 & 12.18 \\
\hline Nigeria & 2000 & 1.48 & 8.55 \\
\hline Nigeria & 2001 & 1.37 & 6.86 \\
\hline Nigeria & 2002 & 1.26 & 5.73 \\
\hline Nigeria & 2003 & 1.05 & 5.06 \\
\hline Norway & 1999 & 7.23 & 14.39 \\
\hline Norway & 2000 & 6.11 & 13.21 \\
\hline Norway & 2001 & 5.91 & 12.13 \\
\hline Norway & 2002 & 5.97 & 11.28 \\
\hline Norway & 2003 & 5.81 & \\
\hline Oman & 1999 & 7.77 & 23.60 \\
\hline Oman & 2000 & 6.31 & 22.63 \\
\hline Oman & 2001 & 6.46 & 21.09 \\
\hline Oman & 2002 & 6.50 & 20.56 \\
\hline Paraguay & 2003 & 9.70 & 43.76 \\
\hline Peru & 1999 & 9.58 & 34.18 \\
\hline Peru & 2000 & 9.50 & 36.78 \\
\hline Peru & 2001 & 9.35 & 36.14 \\
\hline
\end{tabular}


Table A.1.8 (continued)

\begin{tabular}{|c|c|c|c|}
\hline Country name & Year & $\begin{array}{l}\text { Coverage to GDP per } \\
\text { capita ratio }\end{array}$ & $\begin{array}{l}\text { Coverage to deposits per } \\
\text { capita ratio }\end{array}$ \\
\hline Peru & 2002 & 9.15 & 35.95 \\
\hline Peru & 2003 & 8.76 & 37.63 \\
\hline Philippines & 1999 & 2.52 & 4.51 \\
\hline Philippines & 2000 & 2.28 & 4.21 \\
\hline Philippines & 2001 & 2.13 & 4.10 \\
\hline Philippines & 2002 & 1.99 & 3.82 \\
\hline Philippines & 2003 & 1.87 & 3.76 \\
\hline Poland & 1999 & 2.10 & 5.73 \\
\hline Poland & 2000 & 2.30 & 6.30 \\
\hline Poland & 2001 & 2.71 & 6.85 \\
\hline Poland & 2002 & 3.63 & 9.75 \\
\hline Poland & 2003 & 4.98 & 13.58 \\
\hline Portugal & 1999 & 2.31 & 2.33 \\
\hline Portugal & 2000 & 2.17 & 2.19 \\
\hline Portugal & 2001 & 2.04 & 2.10 \\
\hline Portugal & 2002 & 1.94 & 2.14 \\
\hline Portugal & 2003 & 1.92 & 2.12 \\
\hline Romania & 1999 & 1.90 & 16.01 \\
\hline Romania & 2000 & 1.82 & 17.69 \\
\hline Romania & 2001 & 1.70 & 17.09 \\
\hline Romania & 2002 & 1.62 & 13.89 \\
\hline Romania & 2003 & 1.39 & 12.30 \\
\hline Russia & 2003 & 1.08 & 5.16 \\
\hline Serbia \& Montenegro & 2001 & 0.07 & \\
\hline Serbia \& Montenegro & 2002 & 0.05 & \\
\hline Serbia \& Montenegro & 2003 & 0.04 & \\
\hline Slovak Republic & 1999 & 1.94 & 3.49 \\
\hline Slovak Republic & 2000 & 1.91 & 3.26 \\
\hline Slovak Republic & 2001 & 2.08 & 3.48 \\
\hline Slovak Republic & 2002 & 2.77 & 4.83 \\
\hline Slovak Republic & 2003 & 4.25 & 7.39 \\
\hline Slovenia & 2001 & 1.77 & 3.39 \\
\hline Slovenia & 2002 & 1.59 & 3.00 \\
\hline Slovenia & 2003 & 1.84 & 3.38 \\
\hline Spain & 1999 & 1.07 & 1.38 \\
\hline Spain & 2000 & 1.33 & 1.63 \\
\hline Spain & 2001 & 1.26 & 1.49 \\
\hline Spain & 2002 & 1.19 & 1.38 \\
\hline Spain & 2003 & 1.11 & 1.27 \\
\hline Sri Lanka & 1999 & 1.65 & 4.92 \\
\hline Sri Lanka & 2000 & 1.47 & 4.39 \\
\hline Sri Lanka & 2001 & 1.33 & 3.86 \\
\hline Sri Lanka & 2002 & 1.20 & 3.47 \\
\hline Sri Lanka & 2003 & 1.07 & 3.04 \\
\hline Sweden & 1999 & 1.07 & \\
\hline Sweden & 2000 & 1.01 & \\
\hline Sweden & 2001 & 0.98 & \\
\hline Sweden & 2002 & 0.95 & \\
\hline
\end{tabular}




\begin{tabular}{|c|c|c|c|}
\hline \multicolumn{4}{|c|}{ Table A.1.8 (continued) } \\
\hline Country name & Year & $\begin{array}{c}\text { Coverage to GDP per } \\
\text { capita ratio }\end{array}$ & $\begin{array}{c}\text { Coverage to deposits per } \\
\text { capita ratio }\end{array}$ \\
\hline Sweden & 2003 & 0.92 & \\
\hline Switzerland & 1999 & 0.55 & 0.36 \\
\hline Switzerland & 2000 & 0.53 & 0.44 \\
\hline Switzerland & 2001 & 0.52 & 0.43 \\
\hline Switzerland & 2002 & 0.52 & 0.40 \\
\hline Switzerland & 2003 & 0.53 & 0.37 \\
\hline Taiwan & 1999 & 2.37 & \\
\hline Taiwan & 2000 & 2.29 & \\
\hline Taiwan & 2001 & 2.35 & \\
\hline Taiwan & 2002 & 2.31 & \\
\hline Tanzania & 1999 & 1.28 & 9.87 \\
\hline Tanzania & 2000 & 1.16 & 8.34 \\
\hline Tanzania & 2001 & 1.05 & 6.99 \\
\hline Tanzania & 2002 & 0.97 & 5.68 \\
\hline Tanzania & 2003 & 0.88 & 4.88 \\
\hline Trinidad \& Tobago & 1999 & 1.48 & 3.40 \\
\hline Trinidad \& Tobago & 2000 & 1.24 & 3.01 \\
\hline Trinidad \& Tobago & 2001 & 1.13 & 2.81 \\
\hline Trinidad \& Tobago & 2002 & 1.10 & 2.70 \\
\hline Trinidad \& Tobago & 2003 & 1.02 & \\
\hline Turkey & 2002 & 12.59 & 59.39 \\
\hline Uganda & 1999 & 7.98 & 68.43 \\
\hline Uganda & 2000 & 7.48 & 57.91 \\
\hline Uganda & 2001 & 6.87 & 54.72 \\
\hline Uganda & 2002 & 6.89 & 44.15 \\
\hline Uganda & 2003 & 6.50 & 40.34 \\
\hline Ukraine & 1999 & 0.46 & 4.87 \\
\hline Ukraine & 2000 & 0.35 & 3.18 \\
\hline Ukraine & 2001 & 0.29 & 2.30 \\
\hline Ukraine & 2002 & 0.26 & 1.55 \\
\hline Ukraine & 2003 & 0.27 & 1.19 \\
\hline United Kingdom & 1999 & 1.30 & \\
\hline United Kingdom & 2000 & 1.24 & \\
\hline United Kingdom & 2001 & 2.07 & \\
\hline United Kingdom & 2002 & 1.97 & \\
\hline United Kingdom & 2003 & 1.89 & \\
\hline United States & 1999 & 3.02 & 10.57 \\
\hline United States & 2000 & 2.89 & 10.18 \\
\hline United States & 2001 & 2.85 & 9.16 \\
\hline United States & 2002 & 2.78 & 8.67 \\
\hline United States & 2003 & 2.67 & 8.36 \\
\hline Venezuela & 1999 & 1.52 & 10.02 \\
\hline Venezuela & 2000 & 1.17 & 8.00 \\
\hline Venezuela & 2001 & 1.08 & 6.98 \\
\hline Venezuela & 2002 & 2.29 & 16.51 \\
\hline Venezuela & 2003 & 1.87 & 10.30 \\
\hline Vietnam & 2000 & 5.33 & \\
\hline Vietnam & 2001 & 4.96 & \\
\hline
\end{tabular}


Table A.1.8 (continued)

\begin{tabular}{|c|c|c|c|}
\hline Country name & Year & $\begin{array}{c}\text { Coverage to GDP per capita } \\
\text { ratio }\end{array}$ & $\begin{array}{c}\text { Coverage to deposits per } \\
\text { capita ratio }\end{array}$ \\
\hline Vietnam & 2002 & 4.51 & \\
\hline Vietnam & 2003 & 4.03 & \\
\hline
\end{tabular}




\section{A.2 Details on deposit insurance schemes for each country}

In this section we provide details on the deposit insurance schemes in each country along with sources and the relevant laws and names of the governing institutions wherever available. The sources referenced here are listed with details below in section A.3.

Albania. (Albanian Deposit Insurance Agency, Law No. 8873, Law on the Insurance of the Deposits) The explicit deposit insurance scheme (EDIS) of Albania was established in March 2002. It has an official administration by the Deposit Insurance Agency. The membership for the fund is compulsory and is contributed by both the state and the banks, where the premiums are not risk-adjusted and they are $0.5 \%$ of the average insured deposits. The deposits of up to 350,000 lek are fully insured, and $85 \%$ of 350,000 to 700,000 lek is insured.

Sources: Bank of Albania. (2002), IADI Member Profiles: Albanian Deposit Insurance Agency.

Algeria. (Bank of Algeria) The deposit insurance mechanism was established in December 1997. The maximum coverage is 600,000 Algerian dinars per depositor per institution and it has not changed since establishment as of 2004. The system was introduced in response to the expansion of the banking sector with the start up of many private national and international banks. Prior to this date, all the deposit banks were owned by the state (the treasury) with an implicit blanket guarantee.

Source: Own survey of deposit insurers.

Argentina. (SEDESA, Law 24, 485) Before 1979 deposits were unconditionally guaranteed by the Argentinean government. In 1979 an explicit system of deposit insurance scheme was established by the military government. The scheme was optional for private banks and required the insured banks to make contributions to the fund. The central bank provided full coverage for the first million pesos (about \$640) and ninety percent thereafter. Later in 1991 the scheme was abolished and substituted by a more transparent supervision. In April 1995, an insurance scheme was re-introduced following the suspension of five private banks by the government. The scheme (SEDESA) covers all types of deposits except ones that pay more than 200 basis points above the reference rate. Membership to the system is compulsory. The scheme has private administration. Current accounts, savings accounts and time deposits are covered up to $\$ 30,000$. The initial coverage limit of the system was $75 \%$ of 100 Million $\$ A$ rg. This limit was reduced to $75 \%$ of 81,000 \$Arg. The monthly premiums for banks are $0.015 \%$ to $0.06 \%$ of deposits. Additional assessments set by the central bank are also made based on a bank's risk evaluation. The deposits of foreign branches of Argentine banks are not subject to the scheme and deposits of foreign bank branches in Argentina are subject to the scheme. Sources: Garcia (1999), Institute of International Bankers (1999), Kyei (1995), Miller (1993), Oxford Analytica Brief (1995), SEDESA (2003), Talley and Mas (1990).

Austria. (Deposit Guarantee Fund, Credit System Act) The Deposit Guarantee Fund was established in 1979 and was revised according to the EU directives after 1995. The system has private administration. Funding is ex-post. Government bonds may be issued when necessary. Initially the coverage limit was ATS 200,000 and it was raised to ATS 260,000 in April 1996 following Austria's entry to the EC. After the ATS/EUR parity was fixed, the sum was slightly adjusted to ATS 275,000 in 1999 since the Euro became legal tender in Austria amounting to EUR 20,000. Deposits of the government, large corporations, insiders and criminals are excluded. The deposits of natural persons are covered in full up to the coverage limit, whereas deposits of non-households are covered only up to $90 \%$ of the limit, where the maximum coverage is calculated per depositor per institution (i.e. two or more accounts of the same person in one bank is treated like a single deposit). Unlimited coverage never existed in Austria.

Sources: Own survey of deposit insurers, Garcia (1999), Kyei (1995).

Bahamas. (Protection of Depositors Act, Deposit Insurance Fund) The Deposit Insurance Fund and the Deposit Insurance Corporation were established in November 1999 after the passing of the Protection of Depositors Act in September 1999. The scheme is legislated by the government and administered by a Board of Management of six members appointed by the Minister of Finance. Membership is mandatory. Domestic deposits in Bahamian dollars including saving and checking accounts, certificates of deposit, guaranteed investment certificates, travelers checks, money orders, and certified drafts of checks are covered up to 50,000 Bahamian dollars, which has not changed since establishment. The coverage limit is applied per depositor per institution and coverage was never unlimited. There is no co-insurance. The scheme is privately funded by flat rate premiums fixed at 120 of $1 \%$ assessed on insured deposits. 
Sources: Own survey of deposit insurers, IADI Survey: Bahamas (2003).

Bahrain. (Deposit Protection Scheme and Deposit Protection Board, Resolution No. 3) The deposit protection scheme of Bahrain came into effect in November 1993. The scheme has joint administration and ex-post funding. Both resident and non-resident deposits with Bahrain offices of full commercial bank are covered. The coverage is extended to the lesser of BD 15,000 and three quarters of the total eligible deposits of the depositor in the liquidated commercial bank. The scheme extends coverage to both local and foreign currency deposits. The excluded deposits are; government, illegal, inter-bank, deposits of affiliates, shareholders, directors and officers of the banks.

Sources: Bahrain Monetary Agency (2004), Garcia (1999).

Bangladesh. (Deposit Insurance Fund, Deposit Insurance Ordinance 1984) The deposit insurance scheme of Bangladesh was established in 1984. The system excludes the deposits of domestic and foreign governments, banks and other financial institutions. Deposits in foreign currencies are not covered. All scheduled banks are obligated to be members of the scheme and pay a premium on their deposits at a rate of $0.5 \%$. The system is jointly administered and financed. The agency's finances are co-mingled within the central bank.

Sources: Asiatic Society of Bangladesh (2004), Garcia (1999), Kyei (1995).

Belarus. (The Guarantee Fund for the Protection of Deposits by the Population) The deposit insurance system in Belarus was established in 1996 and went through several revisions since then. In 1998, the government promised full guarantees for banks authorized to provide service to government programs. Then they were taken under the supervision of the National Bank of Belarus (NBB) in 2000. In 2001 NBB issued further rules about insurance for ruble and foreign currency deposits in non-authorized banks. As of 2003 the equivalent of USD 2000 (per person per bank) were fully covered under the insurance scheme, whereas $80 \%$ coverage was provided for the next USD 3000 (that is from USD 2000 to USD 5000). Different groups of banks receive different treatment. For example, two large authorized banks do not pay insurance premiums to the Guarantee Fund and their deposits are implicitly covered by the government. On the other hand, the group of banks other than those "authorized" by the government are subject to the coverage limit indicated and are covered by the Guarantee Fund only.

Sources: Barth, Caprio and Levine (2004), Research Center of the Institute for Privatization and Management (2003).

Belgium. (Rediscount and Guarantee Institute, Royal Order 175 and March 1982 Legislation) Before 1995 there were two separate funds (one for banks and one for private savings institutions) that were managed by the institute. Membership was not mandatory. After the changes made in 1995, all institutions are required to participate in the system and there is now only a single fund that covers all credit institutions. In 1995 the coverage limit of 500,000 Belgian Francs was changed to ECU 15,000, which was later replaced by a limit of EUR 20,000 in year 1999. If the funds' liquid assets fall below a critical level, the premiums paid by the banks can be raised by a maximum of $0.04 \%$. The state can provide a limited guarantee.

Sources: Bruyneel and Miller (1995), Garcia (1999), Kyei (1995).

Bolivia. (Fund for Financial Restructuring) The deposit insurance scheme in Bolivia was founded in December 20, 2001. It is governed by the Financial Restructuring Fund that acts as a deposit insurer. Membership is compulsory by all financial institutions and until 2005 the Central Bank was the responsible party before the Fund gets fully capitalized. The premiums are proportional to private sector deposits. Before 2005 the deposits were covered up to 50 percent of the privileged obligations, although there does not exist a maximum amount yet. For example, in terms of the order of obligations, private sector deposits, judicial deposits, and other obligations to the private sector come in first priority. The coverage is extended to foreign currency deposits as well.

Source: Ioannidou and Dreu (2004).

Bosnia-Herzegovina. (Deposit Insurance Agency of the Federation of Bosnia and Herzegovina) The deposit insurance scheme was established in 1998 by the Law on Deposit Insurance published in the Official Gazette No. 41/98. The system is legislated and administered by the government. The membership to the scheme is mandatory and banks need to pay $0.3 \%$ ( $0.5 \%$ until July 2001) of total deposits per year as insurance premiums on a quarterly basis. The deposits are covered up to KM 5000 without any coinsurance 
and they are granted on a per depositor per institution basis. The scheme is privately funded and it extends to foreign currency and inter-bank deposits as well.

Sources: Own survey of deposit insurers, IADI Survey: Bosnia and Herzegovina (2002).

Brazil. (Fundo Garantidor de Creditos-FGC, Resolutions 2197, 3024) FGC commenced its operations in November 1995 . The scheme is privately administered. Membership to the system is mandatory. The banks pay a premium of $0.3 \%$ of the insured deposits. The system does not extend coverage to inter-bank deposits and the coverage limit is set at Reais 20,000. The EDIS was revised in 2002 but the coverage was left unchanged.

Sources: FGC (2004), Garcia (1999), Talley (1998).

Bulgaria. (Law on Bank Deposit Guaranty, Deposit Insurance Fund) The deposit insurance scheme in Bulgaria was established in January 1996 based on Directive 94/19/EC of 1994. At the time, only deposits of physical persons were insured up to EUR 2,500. Due to the failure of 15 banks in the 1996 financial crisis, a blanket guarantee was applied to individual deposits and 50\% repayment on company deposits. The Bulgarian Deposit Insurance Fund was established in early 1999. The coverage was raised to BGN 6,900 (EUR 3,528) in April 1998, to BGN 10,000 (EUR 5,113) in 2001 and finally to BGN 15,000 (EUR 7,670 ) in 2002 . The scheme is jointly administered and the membership is mandatory. Insider deposits and deposits paying preferential interest rates are not covered. If the funds' resources are not adequate, banks can be called to contribute an advance premium of $1.5 \%$ of insured deposits. The co-insurance was abolished in 2001. The fund has the right to borrow, including from the government in the last resort to receive donations and foreign assistance.

Sources: Central European (1998), Own survey of deposit insurers, Garcia (1999), Law on Bank Deposit Guaranty-Bulgaria (1998).

Cameroon, Chad, Central African Republic, Gabon, Equatorial Guinea, and Republic of Congo. A proposal was drafted in 1999 but only ratified by two out of these six CEMAC countries. Thus, they are not considered to have deposit insurance systems unlike earlier sources Demirgüç-Kunt and Sobaci (2000) and Garcia (1999). These African countries share a common central bank. The features of the proposed system are as follows: mandatory membership, joint administration, a permanent fund in place, exclusion of deposits of foreign currencies. The assessment bases for the premiums are deposits and non-performing loans, and the premium rate is $0.15 \%$ of deposits plus $0.5 \%$ net non-performing loans. When necessary, budgetary resources will be available from member countries.

Sources: Own survey of deposit insurers, Garcia (1999).

Canada. (Canada Deposit insurance Corporation, Deposit Insurance Corporation Act of Canada) The deposit insurance scheme in Canada was established in 1967 and the initial coverage was \$Can 20,000. In 1983 the deposit coverage was raised to $\$$ Can 60,000 , while retirement accounts and deposits held in trust received separate protection with an additional \$Can 60,000. The scheme applies to the aggregate amount held per depositor per institution. The system is jointly administered and the membership is compulsory. The covered deposits are savings and demand deposits, term deposits such as guaranteed investment certificates and debenture issues by loan companies, money orders, drafts, checks, and traveler's checks issued by member institutions. The fund can borrow from the markets and the government, but is charged at private market rates.

Sources: Own survey of deposit insurers, Garcia (1999), Kyei (1995).

Chile. (Superintendent of Banks, Banking Law) The DIS of Chile was established in 1986. The system does not have a permanent fund in place. The Chilean Central Bank guarantees $100 \%$ of the demand deposits in full, and $90 \%$ of the household savings and time deposits up to 120 Unidades de Fomento (1 Unidad de Fomento $=$ US $\$ 24$ as of May 2003) per person. The central bank is responsible for demand deposits. Banks with demand deposits in excess of 2.5 times the capital reserves are required to maintain a $100 \%$ marginal reserve requirement in short-term central bank or government securities lined to the central bank. The coverage is extended to foreign currency deposits as well and there is no distinction regarding the type of depositor.

Sources: Garcia (1999), IADI Survey: Chile (2003), Kyei (1995). 
Colombia. (Financial Institution Guarantee Fund, Banking Law 117 of 1985) The deposit insurance scheme of Colombia was established in 1985. The scheme is government legislated and administered and membership to the system is mandatory. Deposits in foreign currencies are excluded, whereas inter-bank deposits are not. The coverage is per depositor per institution up to 20 million Colombian pesos with a $25 \%$ co-insurance. The general premium rate is currently $0.5 \%$ on all deposits which will go down to $0.3 \%$ after January 2007.

Source: IADI Survey: Colombia (2002).

Croatia. (State Agency for Deposit Insurance and Bank Rehabilitation) The deposit insurance scheme of Croatia was established in 1997. Even though the system is privately administered, some decisions must be approved by the central bank. Inter-bank deposits are not covered. The scheme extends coverage to deposits in foreign currencies, except to foreign currency deposits placed prior to 1993, which were covered by a government bond issue. The fund may borrow form the central bank.

Sources: Garcia (1999), Kyei (1995).

Cyprus. (Deposit Protection Scheme, Central Bank of Cyprus) The deposit protection scheme of Cyprus came into effect on September $1^{\text {st }}$, 2000. It is jointly administered by the Central Bank and Management Committee. Membership is mandatory for the scheme and it covers all deposits except entities or persons against which criminal proceedings have been instigated or which a confiscation order has been made. The coverage limit is $90 \%$ of the Cyprus pound equivalent of EUR 20,000 per depositor per institution.

Source: IADI Survey: Cyprus (2003).

Czech Republic. (Deposit Insurance Fund, Act No 156, 1994) The Deposit Insurance Fund of the Czech Republic was established in December 1994. It is government legislated and privately administered. The insurance is granted for savings and checking accounts as well as certificates of deposit and foreign currency deposits; whereas, promissory notes, inter-bank deposits and other securities are not covered. The scheme covers $90 \%$ of all insured deposits up to the equivalent of EUR 25,000 per depositor per institution. Membership to the Fund is compulsory and the premium rates are $0.1 \%$ of all insured deposits including accrued interest for banks; whereas $0.05 \%$ for building savings banks. With regards to the government participation in funding, a law (no 156/1994) mandates that the state will provide 50\% of the funds needed for compensation of depositors by the DIF. The central bank and the government would equally make loans to cover any shortfall in funding.

Sources: Garcia (1999), IADI Survey: Czech Republic (2003), Institute of International Bankers (1997), Kyei (1995).

Denmark. (Deposit Guarantee Fund, Act 850, 1987; Order 118, 1988) The Guarantee Fund of Denmark was established in 1987. The system is government legislated and privately administered. The fund can borrow from banks with a possible guarantee from the government. The maximum coverage limit of DKK 250,000 was raised to DKK 300,000 (about EUR 40,000) effective September 1995. The fund covers registered deposits net of loans and other liabilities of the depositor vis-à-vis the bank per depositor per institution and membership to the fund is mandatory. Certain accounts established according to law such as pension accounts, children's saving accounts and attorneys' client accounts are covered in full.

Sources: Own survey of deposit insurers, IADI Survey: Denmark (2002), Institute of International Bankers (1996).

Dominican Republic. (Savings Account Insurance, National Housing Bank Law) The deposit insurance scheme of Dominican Republic was established in 1962 and it only covers the savings and loan associations. Membership to the system is not compulsory. The system is jointly administered and funded. The government can fund the DIS through savings and loan associations. Foreign currencies are covered, whereas inter-bank deposits are not.

Sources: Garcia (1999), Kyei (1995).

Ecuador. (Deposit Guarantee Agency) The DIS in Ecuador was established on December $3^{\text {rd }}, 1998$ after a major financial crisis and failure of the biggest bank Filanbanco. The system is government legislated and administered. It excludes the deposits of owners, current or recent directors or managers. The fund can borrow, but it is not clear from whom. Both inter-bank and foreign currency deposits are covered. The 
coverage was initially full and in 2001 it was changed to four times the per capita GDP, which was still in existence as of 2003.

Sources: Garcia (1999), IADI Survey: Ecuador (2003).

El Salvador. (Deposit Guarantee Institution, Bank Law 1999) The Guarantee Institution of El Salvador was established in November 1999 and the system is government legislated and administered. Initial funding was provided through the central bank which is later augmented by premiums collected at a $0.1 \%$ annual rate on total deposit liabilities from the members. The membership is mandatory and the coverage limit was an equivalent of $\$ 6,700$ (approximately Colon 58,424) as of 2003 . The previous limits were $\$ 4000$ (Colon 35,000) in 1999 and $\$ 6286$ (Colon 55,000) in 2000. Only savings and checking accounts are eligible for coverage.

Sources: Own survey of deposit insurers, IADI Survey: El Salvador (2003).

Estonia. (Deposit Guarantee Fund) The deposit insurance system of Estonia was established in October 1998. The system initially guaranteed 20,000 Estonian kroons (EEK), which was raised to EEK 40,000 as of January 1, 2000. The Guarantee Fund Act entered into force on July $1^{\text {st }}, 2002$ and set coverage levels at EEK 40,000 initially to be raised to EEK 100,000 on December $31^{\text {st }}, 2003$, then to EEK 200,000 on December $31^{\text {st }}, 2005$ and EUR 20,000 starting on December $31^{\text {st }}, 2007$ the latest. The initial funding was granted by the government and banks paid EEK 50,000 at the start-up. The fund can borrow without a government guarantee or ask the government to borrow a limited amount on its behalf. The types of deposits not covered are deposits in foreign currencies, deposits of insiders, money-launderers, governments at all levels, larger businesses, financial institutions including insurance companies, other members of the same corporate group, and those that pay "substantial higher rates". The coverage amount is calculated per depositor per institution.

Sources: Own survey of deposit insurers, Garcia (1999), U.S. Embassy Reports (1998).

Finland. (Deposit Guarantee Fund, Act on Credit Institutions) The DIS of Finland was established in 1969. In 1998 it was revised in accordance with the EU directives. Before the changes, deposits were covered in full. In the new system a maximum limit of 150,000 FIM was set for the coverage limit. Currently, the coverage is up to EUR 25,000 per depositor per institution. The scheme is privately administered by the member banks in compliance with the rules prescribed by the Ministry of Finance and supervised by the FSA. Foreign currency deposits are covered. Deposits of the central bank and credit institutions are excluded. The government and central bank have borne losses in the past. Membership to the Fund is mandatory and the premium has a fixed $0.05 \%$ fixed part and a variable part based on solvency which can be at maximum $0.25 \%$.

Sources: Garcia (1999), IADI Survey: Finland (2002), Institute of International Bankers (1998), Kyei (1995), Valori and Vesala (1998).

France. (Fonds de Garantie des Depots) The DIS of France was established in 1980 and revised in 1986. It is an unfunded scheme in which the banks contribute to the fund on demand. There are separate schemes for commercial banks, and for mutual savings and cooperative banks. The system is privately administered and jointly funded. Debt securities insured by institutions, deposits of the central government, insiders, affiliated companies and money launderers are excluded from coverage. Initially, coverage was at 200,000 FF and after 1986 it was raised to 400,000 FF. In 1999, according to regulation 99-05, the limit was finally set at EUR 70,000 per depositor per institution. Coverage extends to foreign deposits as well and there is no co-insurance.

Sources: Own survey of deposit insurers, Fonds de Garantie des Depots (1999), Garcia (1999).

Germany. (Deposit Security Fund, Savings Bank Security Fund and Credit Cooperation Security Scheme, Federal Association of German Banks) The first nation-wide joint fund operated by private banking sector in Germany was established in 1966 by the Federal Association of German Banks. The fund protected savings, salary, and pensioners' accounts up to DM 10,000 and other sight and time deposits of natural persons up to DM 20,000. In 1974, the coverage was enlarged to cover up to $30 \%$ of the equity capital per depositor, which is still binding in terms of the private Fund. There are separate schemes by the German Savings Bank, Giro Association, and credit cooperative banks (the latter dates back to 1930s to the aftermath of the Great Depression). These guarantee funds aim at protecting the institutions themselves and hence, provide indirect protection to depositors as a by-product. In 1994, a voluntary deposit protection 
fund was established by the public-owned banks. In line with the transformation to EC Deposit Guarantee Directive, the official binding statutory deposit protection has been limited to $90 \%$ of EUR 20,000 for commercial banks, which co-exists with the voluntary funds by various banking associations. In the official and voluntary deposit protection schemes, coverage amounts are calculated as per depositor.

Source: Own survey of deposit insurers.

Gibraltar. (The Deposit Guarantee Scheme Ordinance) The deposit insurance scheme in Gibraltar was established in 1998 in line with the insurance arrangements in the EU via directive 94/19/EC. It is jointly administered and privately funded, where the membership is mandatory. There is no permanent fund in place. The banks make ex-post contribution to the fund and pay administrative expenses on a regular basis. The coverage is the lesser $90 \%$ of all qualifying deposits or 18,000 British pounds (or Sterling equivalent of EUR 20,000, whichever is the greater).

Sources: Own survey of deposit insurers, Garcia (1999), Gibraltar Deposit Guarantee Board (1998).

Greece. (Hellenic Deposit Guarantee Fund, Law 2832/2000) The deposit guarantee scheme of Greece was established in 1995 by Law 2324/95 which was later amended by 2832/2000. It is administered jointly. The board has eight members; three members from the Bank of Greece, five members from the Hellenic Bank Association with a participant from the Ministry of Finance. If the fund resources are not sufficient to meet the depositors' claims, members may be called upon to pay an additional contribution that can not exceed $300 \%$ of the last annual contribution. The premiums paid by the members are determined by the following brackets: 0.5 million GRD - 1.25\%, 51-250 million GRD - 1.20\%, 251-750 million GRD - 1.175\%, 7511750 million GRD $-0.205 \%, 1751$ million GRD and above - 0.025\%. Inter-bank, insider, illegal and central government deposits are not covered. Membership is mandatory by the commercial and cooperative banks and the Fund covers savings, checking and foreign currency deposits. The coverage limit is EUR 20,000 per depositor per institution with no co-insurance.

Sources: Garcia (1999), Hellenic Deposit Guarantee Fund (2000), IADI Survey: El Salvador (2002), Institute of International Bankers (1996), Kyei (1995).

Guatemala. The deposit insurance scheme in Guatemala was established in 1999 and is publicly governed. A private fund is employed although the government may make temporary contributions with the provision of repayment. The premium rates are set at $1 \%$ plus $0.5 \%$ when the fund falls short of the target. The coverage limit is $\$ 2,800$ per depositor which can be adjusted to cover between 90 to $95 \%$ of the accounts.

Source: Garcia (2000).

Honduras. The deposit insurance scheme of Honduras was established in 1999 as a response to a major banking crisis and under the Temporary Law of Financial Stabilization, all bank deposits received a full expost guarantee which remained valid until 2002. After September 2003 the government insurance covered insured deposits up to 165,000 lempiras $(\$ 9,500)$. The scheme is publicly administered and jointly funded requiring premiums up to $0.25 \%$ of insured deposits.

Sources: Industry Canada (2002), Garcia (2000).

Hong Kong. (Hong Kong Monetary Authority, Companies Ordinance) There is no explicit deposit insurance scheme in Hong Kong but is soon expected to be introduced. The coverage limit of the proposed scheme is HK\$100,000 (or $\$ 12,820$ ) per depositor per institution. An alternative scheme where small depositors receive a priority treatment is currently in place based on Companies Ordinance that took effect in 1995. The priority limit is the first HK\$100,000 of net deposits.

Sources: Own survey of deposit insurers, IADI Survey: Hong Kong (2003).

Hungary. (National Deposit Insurance Fund, Act XXIV of 1993) The deposit insurance fund of Hungary was established on $31^{\text {st }}$ March, 1993. The system is government legislated and privately administered. Members of the board of directors are, the president of the National Bank of Hungary, the administrative secretary of the state of the Ministry of Finance, the president of inspections, two persons delegated by the interest-representing organizations of financial institutions, and the managing director of the DIF. Deposits of government, insiders, professional investors, money launderers, and other banks are excluded from coverage. The government can guarantee fund borrowing from the central bank or private markets if requested. Membership to the Fund is compulsory. The coverage is mainly extended to savings accounts, certificates of deposit and foreign currency deposits. However, only currencies denominated in EUR or 
other OECD countries are insured. The coverage limit was initially HUF 1 million (approximately $\$ 3700$ ), which was raised to HUF $3,222,222$ on January $1^{\text {st }}, 2003$ and to HUF $6,555,555$ on May $1^{\text {st }}, 2004$. The maximum coverage is calculated per depositor per institution.

Sources: Own survey of deposit insurers, Garcia (1999), IADI Survey: Hungary (2003), Kyei (1995), and Ministry of Finance of Hungary (1993).

Iceland. (Deposit Insurance Fund for Savings Banks; Deposit Insurance Fund for Commercial Banks, Acts 86, 87/1985; Depositors' and Investors' Guarantee Fund 98/1999) The deposit insurance system of Iceland was first established in 1985. There were separate schemes for commercial banks and savings banks which were monitored by the supervisory agency. The fund for the banks had a member of the government on its board. Even though the coverage in principle was full, the system was considered to have a co-insurance mechanism due to the fact that above the minimum coverage limit of ECU 20,000, the actual compensation of depositors were determined according to the resources of the fund, which received no public support. Act 98/1999 established the new scheme and the Fund in accordance with the EU directives since Iceland is a member of the European Economic Area (EEA). The new fund took over the assets of the previous two funds, and it is both privately established and administered. The Central Bank provides such services as accounting and bookkeeping as well as securing valuable documents. The membership to the Fund is mandatory. The coverage limit was ISK $1,700,000$ which is tied to the EUR exchange rate as of January $5^{\text {th }}$, 1999 (approximately EUR 21,000) and hence, is worth ISK 2,091,000 as of 2003. Coverage is extended per depositor.

Sources: Own survey of deposit insurers, Garcia (1999), IADI Survey: Iceland (2003), Kyei (1995).

India. (Deposit Insurance and Credit Guarantee Corporation (DICGC), DICGC Act, 1961) The DIS of India was established in 1962 following two bank failures in 1961. Initially the system covered exclusively the commercial banks. In 1968 cooperative bank with a minimum size operating in states having pertinent legislation was included in the system. In 1975 coverage was extended to rural banks as well. The coverage limits have been changed in time as follows: initially Rs 1,500; Rs 5,000 in 1968; Rs 10,000 in 1970; Rs 20,000 in 1976; Rs 30,000 in 1980, and Rs 100,000 since May $1^{\text {st }}, 1993$. The system is administered officially. Certificates of deposits, government, inter-bank, and illegal deposits are not covered.

Sources: Deposit Insurance and Credit Guarantee Corporation-India (2004), Garcia (1999), Kyei (1995), Talley and Mas (1990).

Indonesia. There exists a full blanket guarantee in Indonesia since 1998 in response to the banking crisis. The government is planning to offer an explicit, limited, and self-funded deposit insurance scheme.

Source: IMF Factsheet (1998).

Ireland. (Deposit Protection Account, Central Bank, Central Bank Act, 1989) The Irish DIS was established in 1989. The system is administered officially. Public funding may be available through central bank and government support with parliamentary approval. Initially $80 \%$ of the first 5000 pounds, $70 \%$ of he next 5000 pounds, and $50 \%$ of the next 5000 pounds was covered. In July 1995 the coverage limit was set at ECU 15,000 . Currently it is at $90 \%$ of EUR 20,000. The system does not extend coverage to certificates of deposits, deposits of major owners and senior managers, and money launderers.

Sources: Garcia (1999), Institute of International Bankers (1996), Kyei (1995).

Isle of Man. (Financial Supervision Commission, Banking Business Regulations-Compensation of Depositors, 1991) The scheme came into effect on August $14^{\text {th }}, 1991$ and it is officially legislated and administered. The maximum coverage per depositor per institution is the lesser of 15,000 pounds or $75 \%$ of the deposit amount. The insurance covers saving and checking accounts, certificates of deposit and foreign currency deposits. There is no permanent fund and the funding required by participants in the event of a claim is the greater of 25,000 pounds or the sum of $0.125 \%$ of the average sterling and foreign currency deposits subject to a maximum of 250,000 pounds.

Sources: Own survey of deposit insurers, IADI Survey: Isle of Man (2002).

Italy. (Inter-bank Deposit Protection Fund) The DIS of Italy was established in 1987 and in 1996 the EU Directive $94 / 19$ was accepted. There were separate systems for banks and cooperative institutions initially. Even though the scheme is privately established and administered, we consider it to be jointly administered, due to the fact that most decisions must be approved by the central bank. Criminal, government, insider, 
inter-bank and bearer deposits are not covered. The Bank of Italy can make low-interest rate loans to facilitate a large pay-out. The coverage has been ITL 200 millions per depositor since establishment, which corresponded to EUR 103,291 as of 2003.

Sources: Own survey of deposit insurers, Garcia (1999), IADI Survey: Italy (2003), Kyei (1995).

Jamaica. (Deposit Insurance Corporation, Deposit Insurance Act 1998) The deposit insurance system of Jamaica was established in 1998. It is government legislated and administered. Membership to the scheme is mandatory. Insurance coverage limit was initially J\$200,000 and was raised to J\$ 300,000 after July 2001. Coverage is calculated per depositor per institution and it extends to foreign currency deposits as well.

Sources: Own survey of deposit insurers, IADI Survey: Jamaica (2003).

Japan. (Deposit Insurance Corporation-DIC, Deposit Insurance Law) There are two separate deposit insurance schemes in Japan; one for commercial and Shinkin banks, credit cooperatives and labor and credit associations, and another for agricultural and fishery cooperatives. The first scheme covers demand and time deposits in domestic currency. The coverage was 1 million yens in 1971, 3 millions in 1974, and 10 millions in 1986 covering the principal only; and, it became 10 millions for principal plus interest in 2001. Due to a law amendment in 2002, special deposits for settlement and payment uses have been fully covered. The blanket guarantees were offered for current, ordinary and special deposits in 1996 as well again as a temporary measure. The coverage is otherwise per depositor per institution. The system is government legislated and administered. The government and the central bank provided the initial capital. The fund can borrow from the central bank, and the government can guarantee the DIC's debt. Membership to the Corporation is mandatory. Between 1996 and 2000, banks were required to pay a special premium of $0.0036 \%$ in addition to their regular rate of $0.0048 \%$. As a result of an amendment to the Deposit Insurance Law in February 1998, the government allocated 17 trillion yens to a special account in the DIC.

Sources: Own survey of deposit insurers, Garcia (1999), IADI Survey: Japan (2003), Kyei (1995), Oxford Analytica Brief (1997).

Jordan. (Deposit Insurance Corporation) The DIC of Jordan was established in September 2000 and it is government legislated and administered. Membership to the scheme is compulsory and insurance has been provided up to JD 10,000 since establishment. The coverage is calculated per depositor per institution. Insurance premiums are assessed on a flat basis with a rate of $0.25 \%$.

Sources: Own survey of deposit insurers, IADI Survey: Jordan (2002).

Kazakhstan. (Deposit Insurance Fund) The Deposit Insurance Fund of Kazakhstan was established in November 1999. The system is government legislated and administered. The National Bank of Kazakhstan provided the initial capital of KZT 1 billion for the Fund and its membership became compulsory after January 2004. Member banks pay a flat rate of $0.25 \%$ for two years after they enter the system and $0.16 \%$ from then on. In case of insufficiency, the Fund can borrow from the National Bank and the government. The coverage limit is KZT 400,000 (about $\$ 3000$ as of 2003) per depositor per bank and before 2003 it was KZT 200,000. Foreign currency deposits are also covered.

Source: IADI Member Profiles: Kazakhstan Deposit Insurance Fund, Kazakhstan Deposit Insurance Fund (2004).

Kenya. (Deposit Protection Fund Board, Banking Act No. 17, 1985) The DIS of Kenya was established in 1985 following four bank failures and it became operational in 1986. The scheme is administered officially and funded jointly. The fund can borrow from the central bank. The board is chaired by the governor of the central bank. The Treasury is represented by a permanent secretary.

Sources: Central Bank of Kenya (2004), Garcia (1999), Kyei (1995).

Korea. (Korea Deposit Insurance Corporation, Bank Deposit Insurance Act 1995, Law no. 5042, the BDIA) The Deposit Insurance Corporation of Korea was established in June 1996 and became operational in January 1997. The scheme is government legislated and privately administered. The coverage limit was initially set at 20 million WON but between November 1997 and December 2002, in response to the financial crisis, the deposits were covered in full. Demand deposits, savings and time deposits, installment deposits and mutual installment deposits, and money in trust with a principal were protected accordingly by the scheme. The types of institutions covered are domestic commercial banks, specialized banks, foreign 
bank branches, development institutions, and credit unions. As of 2003, the coverage limit was 50 million won per depositor per institution. The KDIC is legally authorized to borrow from the government or central bank with the approval of the ministry of finance.

Sources: Garcia (1999), IADI Survey: Korea (2003), Korea Deposit Insurance Corporation (2004), Ko (1997), Kyei (1995).

Latvia. (Law on guarantees for deposits of natural persons) The DIS of Latvia was established in 1998. It is administered officially. Insider deposits and accounts in bank already declared bankrupt or insolvent, or in liquidation proceedings are not covered. The initial coverage limit was 500 Lats. In accordance with the EU standards, this amount will be gradually increased up to 13,000 Lats by the year 2008 according to the following schedule: 500 Lats, until December 31, 1999; maximum 1,000 Lats until December 31 2001; maximum 3,000 Lats until December 31, 2003; maximum 6,000 Lats until Dec 31, 2005; maximum 9,000 Lats until Dec 31, 2007; maximum 13,000 Lats after Jan. 2008. The Bank of Latvia and the government made initial contributions to the fund.

Sources: Bank of Latvia (1998), Garcia (1999), Institute of International Bankers (1997).

Lebanon. (National Deposit Guarantee Institution, Banque du Liban, Law no. 28/67) The deposit insurance scheme of Lebanon was established in May 1967. It is administered and funded jointly. The government matches the premiums paid by the banks. The central bank contributed half of the initial capital. The fund can borrow from the central bank. Initially, the scheme extended coverage only to deposits denominated in Lebanese pounds up to LL 30,000 (approximately $\$ 10,000$ at the time) per depositor per bank. The insurance coverage limit was then raised to LL 250,000 (approx. \$2,874) in 1986; to LL 1 million (approx. \$1,887) in 1988 and to LL 5 million $(\$ 5,688)$ in 1991. The level remained the same since then, corresponding to $\$ 3,3317$ as of 2003 . According to a transitional law lasting from the end of 1991 to the end of 1998, deposits in foreign currencies were also covered up to LL 5 million.

Sources: Banque du Liban (2004), Garcia (1999), Kyei (1995).

Liechtenstein. (Financial Services Authority, Liechtenstein Banking Act of 1992, Art.7; Liechtenstein Bankers Association) In accordance with the EU directives Liechtenstein passed a banking law in 1992 requiring banks to provide guarantees for deposits and investments lodged in them. As a privately administered scheme, Liechtenstein Bankers Association started up the Liechtenstein Bankers Association Deposit Guarantee and Investor Protection Foundation. The insurance scheme covers deposits of private customers up to a maximum of EUR 20,000 or its equivalent in other EEA currencies and CHF. Deposits denominated in other currencies are not covered. The Foundation can meet its obligations up to CHF 300 million as constrained by the contractually agreed maximum contribution amounts by member banks.

Source: Liechtenstein Banking Act (1992).

Lithuania. (Deposit Insurance Fund, Deposit Insurance Law, December 1995) The deposit insurance scheme of Lithuania was established in 1996 based on a law that was voted in the parliament in December 1995 after the failure of two large and popular banks. It is officially legislated and administered. The government provided the initial capital and is committed to cover any shortfall. Membership to the Fund is mandatory and the premium rate for banks and branches of foreign banks is $0.45 \%$ and for credit unions $0.2 \%$ of insured deposits. The deposits are covered $100 \%$ up to LTL 10,000 and $90 \%$ from LTL 10,000 to LTL 45,000 ( $\$ 16,200$ as of 2003) per depositor per institution. Under the new law, the insurance limit is projected to reach a level of EUR 20,000 by 2008 .

Sources: Brance, Kammer and Psalida (1996), IADI Survey: Lithuania (2002), IMF Country Report: Lithuania (2002).

Luxembourg. (Deposit Guarantee Association) The scheme was established in 1989. It is privately administered. There is no permanent fund in place. Banks make ex-post contributions when needed. The coverage limit of LUF 500,000 was raised to $90 \%$ of ECU 15,000 in July 1995 . Since January 2000, it is set at $90 \%$ of EUR 20,000 per depositor per institution. Branches of foreign banks are also members of the system. If a foreign bank is organized under the law of another EU member state, it does not have to participate in the system, but the coverage amount should be equal to that allowed in the Luxembourg system.

Sources: Own survey of deposit insurers, Garcia (1999), Institute of International Bankers (1997), Kyei (1995). 
Macedonia. (Deposit Insurance Fund) The scheme in Macedonia was first established in 1996 under the name Saving Deposit Insurance Fund Inc. as a joint shareholders' company by the banks and savings houses. In July 2000, a single Deposit Insurance Fund came into force. The scheme is administered and funded jointly. The fund can borrow from the central bank or domestic and foreign sources if necessary. Membership to the system is mandatory. Coverage is extended to current accounts and savings deposits of natural persons that are denominated in domestic and foreign currencies. The coverage limits have changed according to the following over time: In 1996, the lesser of $75 \%$ of total savings per depositor or denar equivalent of DM 10,000; in 2000, $100 \%$ of deposits up to EUR 1,500 and $90 \%$ of EUR 1,500 to EUR 7,500; in 2002, $100 \%$ of deposits up to EUR 3,000 and $90 \%$ of EUR 3,000 to EUR 10,000; and since May $2003,100 \%$ of deposits up to EUR 10,000 and $90 \%$ of EUR 10,000 to EUR 20,000. The coverage is calculated per depositor per institution and banks were charged a flat premium of $0.7 \%$ as of 2003 .

Sources: IADI Survey: Macedonia (2002), IMF Country Report: Macedonia (2003), Deposit Insurance Fund Skopje (2004).

Malaysia. Malaysia introduced a blanket guarantee for depositors in December 1997 which came into force in 1998. The scheme was introduced in response to the financial crisis.

Source: Garcia (2000).

Malta. (Deposit Guarantee and Investor Compensation Scheme, Financial Services Authority) The regulation approving the creation of a deposit insurance scheme in Malta became effective on January $3^{\text {rd }}$, 2003. The participants of the scheme were required to initially contribute LM 10,000 each and over five years were expected to contribute a total of minimum LM 1 million in proportion to their holdings of eligible deposits. Membership is mandatory. The scheme is being developed in accordance with EU standards and coverage limit is therefore set at $90 \%$ of a maximum EUR 20,000 per depositor per institution.

Source: Malta Financial Services Authority (2004).

Marshall Islands. (Federal Deposit Insurance Corporation-FDIC, Banking Act) The scheme in Marshall Islands was established in 1975. Membership to the system is voluntary. The system is funded by the contributions of the members only. It is administered officially. The coverage limit is set at US\$ 100,000 .

Sources: Garcia (1999), Kyei (1995).

Mexico. (Bank Savings Protection Fund, Credit Institutions Law; Bank Savings Protection Institute, Bank Savings Protection Law) The deposit insurance scheme of Mexico was established in 1986. It is administered officially. In 1990, Bank Savings Protection Fund was created as a trust within the Central Bank providing unlimited guarantee to all lawful banks. The agency's assets were depleted and the trust had to issue government-backed debt after the 1995 banking crisis. In 1999, Bank Savings Protection Institute was established and the new scheme took on a seven stage transition period from blanket to limited coverage. A coverage limit first started to apply in 2003 at 32,262,340 Mexican pesos (equivalent of 10 million UDIs or investment units which is a monetary unit indexed to price level). In 2004 the maximum coverage dropped to 5 million UDIs (16,762,350 Mexican pesos) and was planned to go down to 400,000 UDIs $(1,365,979$ pesos) in 2005. The coverage amount is calculated per depositor per institution. Membership is compulsory for all banks and the premiums are assessed as minimum $0.4 \%$ of a proxy of total bank liabilities. In the 1999 system the rate was $0.3 \%$ with a $0.5 \%$ maximum plus $0.7 \%$ premium when necessary.

Sources: Own survey of deposit insurers, IADI Survey: Mexico (2003).

Micronesia. (Federal Deposit Insurance Corporation-FDIC, Banking Act) The scheme of Micronesia was established in 1963. It is administered officially. Membership to the system is voluntary. The coverage limit is set at US\$100,000. The fund has borrowed from the Central Bank and the Ministry of Finance.

Sources: Garcia (1999), Kyei (1995).

Netherlands. (Collective Guarantee Scheme) The scheme of Netherlands was established in 1978. There is no permanently maintained fund. The banks make ex-post contributions when needed. Ex-post assessments are made case-by-case based on several items of data reported to the central bank. Assessed bank's portfolio is compared to the portfolio of the failed bank. The contribution amounts are determined after 
consultation with the Bankers Committee. The central bank provides interest-free bridge financing. Deposits of large corporations, other banks, insurance companies and insiders are not covered. Deposits of small enterprises and small foundations along with the deposits of households are protected. Covered types of accounts are current and savings accounts, and bank-registered debt instruments. Deposits at branches of foreign banks established in other EU states are not covered. The coverage limits have historically taken the following values: HFL 25,000 in 1978, HFL 44,000 in 1996 and finally EUR 20,000 since 1998. There is no co-insurance.

Sources: Garcia (1999), Garcia and Prast (2003), Institute of International Bankers (1996), Kyei (1995).

Nicaragua. The deposit insurance scheme of Nicaragua was established in 2001. It provides coverage of up to US\$20,000 per depositor per institution. The system is officially legislated and administered. Membership to the fund is mandatory.

Source: Barth, Caprio, and Levine (2004).

Nigeria. (Nigerian Deposit Insurance Corporation-NDIC, Act No. 22) The scheme of Nigeria was established in 1998 by the military government. The federal government made an initial contribution to the fund and it can still extend loans. $40 \%$ of the Corporation's equity is owned by the Federal Ministry of Finance and Economic Development Inc., and the remaining 60\% is held by the Central Bank of Nigeria. Both the Central Bank and the Ministry of Finance are represented in the board which is chaired by the governor of the Central Bank. All categories of traditional deposits are covered except insider deposits, and deposits that serve as collateral for loans. The coverage per depositor has been N 50,000 (about \$366 as of 2003). There is no co-insurance and membership to the fund is mandatory. The premiums are assessed at a flat rate of $0.94 \%$.

Sources: Alawode (1992), Garcia (1999), IADI Survey: Nigeria (2003), Kyei (1995), Talley and Mas (1990).

Norway. (Deposit Guarantee Fund) The scheme of Norway was established in 1961. There are separate funds for commercial banks and savings banks. Both of these funds are privately administered and jointly funded. In Norway there was actually a guarantee fund for savings banks with voluntary membership in 1921 which became obligatory in 1924, whereas a guarantee fund for commercial banks was first introduced in 1938. However, Norway's guarantee fund at the time is not considered a pure deposit insurance scheme so they had no official explicit deposit insurance until 1961. The government and central bank have borne losses in the past. There are seven members on the boards of the funds. One of the members is from the Central Bank, and the other is from the Banking and Securities Commission. Deposits of other banks and deposits of companies in the same group with the depository bank are excluded. The Commercial Banks Guarantee Fund provided unlimited coverage between 1962 and 1997. Since 1997, the maximum coverage amount allowed has been NOK 2 millions per depositor per institution.

Sources: Own survey of deposit insurers, Garcia (1999), Gerdrup (2003), IADI Survey: Norway (2003), Kyei (1995).

Oman. The scheme of Oman was established in 1995. It is officially administered and jointly funded. The central bank matches half of the member banks' premium contributions. The fund can borrow from the government, central bank and the member banks. Deposits of significant shareholders, directors and senior managers, illegal deposits and the deposit of auditors, parent, subsidiary and affiliated companies are excluded. The coverage is up to RO 20,000 or $75 \%$ of net deposits, whichever is less. The premiums are assessed at a rate of $0.2 \%$ but can range from $0.1 \%$ to $0.3 \%$ over time.

Source: Garcia (1999), Garcia (2000).

Paraguay. The deposit insurance scheme of Paraguay came into force in 2003. The coverage limit was set at 75 times the monthly minimum salary as of 2003.

Source: Barth, Caprio, and Levine (2004).

Peru. (Deposit Insurance Fund, Banking Law 1991) The scheme was established in 1991. It is government legislated and privately administered. The Central Bank and the Treasury have made initial contributions. The Fund may borrow from the Treasury. All types of deposits, except bearer certificates for natural persons and non-profit organizations are covered. The premium is computed based on the maximum amount insured and applies only to deposit of individuals and non-profit institutions. The premiums are risk 
adjusted and they have the following annual rates based on insured deposits for different risk ratings; A: $0.45 \%$, B: $0.60 \%$, C: $0.95 \%$, D: $1.25 \%$, E: $1.45 \%$. The coverage limit in 1991 was S 2500 which has been updated according to the wholesale price index on a quarterly basis. In December 1998 it was raised to S 62,000 and just a month earlier it was only at S 13,836 . The limit was S 68,474 by the end of 2003 . The coverage is calculated per depositor.

Sources: Own survey of deposit insurers, IADI Survey: Peru (2003), Garcia (1999), Kyei (1995).

Philippines. (Philippine Deposit Insurance Corporation-PDIC, Republic Act 3591/7800) The scheme of Philippines was established in 1963. It is government legislated and administered and jointly funded. The government provided the initial capital. The central bank has made loans and borne losses. The government and the central bank are represented on the board. All deposit-taking institutions and corporations authorized to perform banking functions in the Philippines are covered and are obliged to be members of the Fund. The coverage is extended to savings and checking accounts; foreign currency, inter-bank and time deposits on a per depositor per institution basis. The coverage limits in Philippine pesos took the following values historically: 10,000 in 1963, 15,000 in 1978, 40,000 in 1984 and 100,000 since 1992.

Sources: Own survey of deposit insurers, Garcia (1999), IADI Survey: Philippines (2003), Kyei (1995), Talley and Mas (1990).

Poland. (Banking Guarantee Fund, Law on Banking Guarantee Fund, 1994) The Polish deposit guarantee scheme was established in 1995. It is officially administered and jointly funded. The Bank of Poland and the government contributed the initial capital. It excludes the deposits of a bank's significant stockholders, directors, or senior managers, the deposits of the treasury, investment firms, or insurance companies. The treasury also insures some housing savings deposits. The coverage is calculated per depositor per institution. In 1995 the coverage limit was calculated as $100 \%$ of up to EUR 1,000 and $90 \%$ of EUR 1,000 to EUR 3,000. Then, the upper limit in euros (the co-insured part) rose over time as follows; 1997: 4,000, 1998: 5,000, 1999:8,000, 2000: 11,000, 2001: 15,000, 2002: 18,000, 2003: 22,500.

Sources: Own survey of deposit insurers, Garcia (1999), Kyei (1995).

Portugal. (Deposit Guarantee Fund, Decree-Law No. 298/92) The scheme of Portugal was established in 1992 and was revised in 1995. It is officially administered and jointly funded. The Bank of Portugal provided the initial capital to the Fund. In 1999 the coverage for agricultural credit cooperatives has been changed to be equivalent to the coverage for commercial banks. The scheme extends coverage to demand, time and foreign currency deposits, but not to those of insiders or criminals, financial institutions or central and local governments. All credit institutions are mandatory members of the Fund and they pay annual premiums at rates ranging between $0.1 \%$ and $0.2 \%$. Initially, the coverage limit was $100 \%$ of first ECU $15,000,75 \%$ of second ECU 15,000, and 50\% of third ECU 15,000. Since June 1999 the coverage limit is fixed at EUR 25,000 without co-insurance per depositor per institution.

Sources: Own survey of deposit insurers, Garcia (1999), IADI Survey: Portugal (2002), Institute of International Bankers (1999), Kyei (1995).

Romania. (Deposit Guarantee Fund in the Banking System) The Romanian scheme was established in 1996. It is government legislated and funded. The fund can borrow from the state, the central bank and other resources. The government can guarantee the debt. Coverage limit is adjusted annually for inflation and it was ROL 125,222,000 (approx. $\$ 3,841$ ) by the end of 2003. The maximum coverage amount is calculated per depositor per institution and the goal is to attain the EU ceiling requirement of EUR 20,000. Each Romanian bank pays an initial contribution equivalent to the $1 \%$ of its subscribed capital of the domestic banks. Foreign bank branches pay an initial contribution equivalent to $1 \%$ of the subscribed bank capital of the minimum capital provided by a Romanian bank. Premium rates range between $0.3 \%$ and $0.6 \%$ of natural persons' deposits. They are calculated according to a formula that includes measures of solvency, profitability, liquidity, ratio of loans to equity, and risk exposure and can be $1.6 \%$ at maximum. Sources: Own survey of deposit insurers, Garcia (1999), IADI Survey: Romania (2002), Institute of International Bankers (1997).

Russia. (Agency for Restructuring Credit Organizations-ARCO, Deposit Insurance Program, Federal Laws 177-FZ through 182-FZ) The deposit insurance system of Russia was legislated in December 2003. The law only covers deposits of physical persons and hence excludes corporate and inter-bank deposits. The coverage limit in rubles was set at $100 \%$ of deposits up to 20,$000 ; 90 \%$ of deposits between 2,000 and 
25,$000 ; 50 \%$ of deposits over 25,000; total coverage not to exceed 100,000. Currently, the limits are tied to minimal wage (MW) which is at 100 rubles. Thus, the figures above re-expressed in terms of MW become $100 \%$ of deposits up to $20 \mathrm{MWs} ; 90 \%$ of deposits between $20 \mathrm{MWs}$ and $250 \mathrm{MWs} ; 50 \%$ of deposits over 250 MWs; total coverage not to exceed 1000 MWs. The law allows both ex-ante and ex-post funding. ARCO provided the initial capital of Rb 3 billion. The Deposit Insurance Agency is planned to be constructed and take over the administration. The premiums are planned to be no more than $0.15 \%$, and in emergency up to $0.3 \%$ which will go down to formed $0.05 \%$ once the fund reaches $5 \%$ of the insured deposit base.

Sources: Agency for Restructuring Credit Organizations-Russia (2004), OECD (2004).

Serbia and Montenegro. (Agency for Deposit Insurance and Bank Rehabilitation, Bankruptcy and Liquidation, Serbia; Deposit Protection Law, Montenegro) The deposit insurance system of Serbia came into force in 2001. The coverage limit is set at 5,000 dinars per depositor per bank which extends to foreign currency deposits as well. On the other hand, Deposit Protection Law was adopted on July $11^{\text {th }}, 2004$ by Montenegro which provide protection up to EUR 5,000 per depositor per bank and the Fund can increase this amount up to EUR 20,000 depending on its resources and the amount of protected deposits.

Sources: Bank Rehabilitation Agency-Serbia (2004), Own survey of deposit insurers.

Slovak Republic. (Deposit Protection Act, No. 118/1996 to 340/2003) The scheme of Slovak Republic was established in March 1996 and was revised in 2001 in accordance with the EU directive 94/19/EC. The system is jointly administered and funded. The central bank made an initial contribution and may make loans to the fund. Anonymous deposits and deposits of owners, directors and senior managers are not covered. The premium rates for building societies are half of those of commercial banks. Membership is mandatory and premium rates range between $0.1 \%$ and $0.3 \%$. Until 2002, the coverage limit for deposits was thirty times the average monthly salary of previous year as published by the National Statistical Office, whereas the rate with savings banks was sixty-fold. In 2002, the bank deposit limit was raised to forty times the average monthly salary. Finally, in 2003 the coverage limit was set at $90 \%$ of inaccessible deposits not to exceed EUR 20,000.

Sources: Own survey of deposit insurers, Garcia (1999).

Slovenia. (Deposit Guarantee System, Banking Act) The deposit guarantee scheme of Slovenia was introduced in 2001. Between 1991 and the end of 2000 there was an explicit unlimited coverage by the government. The system is government legislated and administered. Funding is provided on an ex-post basis such that banks are not obliged to pay premiums but to invest a minimum $2.5 \%$ of the guaranteed deposits in the Bank of Slovenia bills. The membership to the scheme is mandatory for all deposit taking institutions. The coverage limit was SIT 4.2 million in 2001 which went up to SIT 5.1 million (about \$27 thousand) in 2003. The coverage is calculated per depositor per institution and it is extended to foreign currency deposits as well.

Sources: Own survey of deposit insurers, IADI Survey: Slovenia (2003).

Spain. (Deposit Guarantee Fund, Royal Decree Law 4 \& 18) Spain has separate deposit guarantee funds for its commercial banks (established in 1977), savings banks (established in 1980), and credit cooperatives (established in 1982). The system is government legislated and privately administered. Each fund is jointly administered by their management commissions with eight members. Four members are from the Bank of Spain and the other four are from the member institutions. Deposits of financial institutions, public bodies, and insiders are not covered. Deposits in financial institutions from other EU countries are also covered. Membership to the Spanish scheme by branches of foreign banks - including the EU banks - is voluntary. The central bank can make limited loans to the Fund. The premiums are assessed annually with flat rate. In 2003 the rates were $0.6 \%$ for commercial banks, $0.4 \%$ for savings banks, and $1 \%$ for credit cooperatives. The coverage limits have taken the following values historically: 1977: 500,000 pesetas, 1980: 750,000 pesetas, 1981: 1.5 million pesetas, 1995: 15,000 ecus, 1996: 15,000 euros, 2000: 20,000 euros.

Sources: Own survey of deposit insurers, Garcia (1999), IADI Survey: Spain (2003), Institute of International Bankers (1997), Kyei (1995).

Sri Lanka. The scheme of Sri Lanka was established in 1987. It is officially administered and jointly funded. The central bank provided the initial capital and can advance funds. Membership to the scheme is voluntary. Deposits in foreign currencies are not covered. Deposits of the government, public corporations, 
and other banks are also excluded from coverage. The deposit coverage limit was set at Rs 100,000 in 1987 for private individuals only.

Source: Allrefer.com (2004), Garcia (1999).

Sweden. (Deposit Guarantee Board) The deposit insurance scheme of Sweden was established in January 1996 based on the EU directive. In 1992, Sweden introduced a temporary guarantee of all bank liabilities for that year. This temporary guarantee mechanism was abolished in July 1996. The new system is officially administered and jointly funded. The government has borne losses in the past. The scheme covers saving and checking accounts as well as foreign currency and inter-bank deposits of up to SEK 250,000 (approximately $\$ 34,300$ as of 2003).

Sources: Own survey of deposit insurers, Garcia (1999), IADI Survey: Sweden (2003), Institute of International Bankers (1997).

Switzerland. (Deposit Guarantee Scheme) The deposit guarantee scheme in Switzerland was established in 1984. It is privately administered. The scheme is funded exclusively by the members. There is no permanent fund in place. Banks make ex-post contributions when needed. Membership to the scheme is voluntary. The coverage limit for savings deposits per depositor is currently at CHF 30,000 with no coinsurance.

Sources: Barth, Caprio, and Levine (2004), Garcia (1999), Kyei (1995).

Taiwan. (Central Deposit Insurance Corporation-CDID, Deposit Insurance Act, Article 46, 1985) The DIS of Taiwan was established in 1985. It is officially administered and jointly funded. Membership to the system is compulsory by all deposit-taking financial institutions and the premium rates based on three different levels of risk are $0.05 \% 0.055 \%$, and $0.06 \%$. Initially, the maximum coverage of the scheme was NT\$ 700,000, which has been set at NT\$ 1 million since August 1987. The coverage is calculated per depositor per institution. Apart from CDIC, Taiwan government established the Financial Restructuring Fund to provide blanket guarantees in handling the problem institutions between July $11^{\text {th }}$, 2001 and July $19^{\text {th }}, 2004$ with a provision for a one-year extension.

Sources: Own survey of deposit insurers, IADI Survey: Taiwan (2003).

Tanzania. (Deposit Insurance Board-DIB, Financial Institutions Act, 1991) The deposit insurance system of Tanzania was established in 1991 and became operational in 1994. It is government legislated and administered. The government provided the initial capital. The fund can borrow from the central bank. All types of deposits including inter-bank and foreign currency ones are covered up to TZS 250,000 without any co-insurance. Membership to the Fund is compulsory

Sources: Garcia (1999), IADI Survey: Tanzania (2003).

Thailand. Thailand has been offering a full blanket guarantee on all deposits since 1997, the aftermath of the financial crisis. A scheme is planned to be introduced.

Source: Garcia (2000).

Trinidad \& Tobago. (Deposit Insurance Corporation, Financial Institutions Act 1986) The deposit insurance scheme of Trinidad \& Tobago was established in 1986. It is government legislated, officially administered and jointly funded. The fund can borrow from the central bank. The covered deposit types are demand, savings, and time deposits but not inter-bank and foreign currency deposits. The coverage limit per depositor is TT $\$ 50,000$ which was worth US\$ 14,000 at inception and was worth only US\$ 7,930 as of 2003 due to devaluation over time.

Sources: Garcia (2000), IADI Survey: Trinidad and Tobago (2003).

Turkey. (Saving Deposit Insurance Fund, Decree Law No. 70) The fund in Turkey was established in 1983. Until August 2000 it was administered by the Central Bank and then the administration was transferred to the Banking Regulation and Supervision Agency (BRSA) which is financially and administratively autonomous from the government. It is jointly funded; however, the Fund had to be supported by the government especially after major crises in 1994 and 2000. Initially coverage was extended to deposits and CDs in Turkish Liras, and foreign currency denominated savings accounts of real persons domiciled in Turkey. Currently, deposits, by natural persons that are native or of foreign origin, in the forms of domestic currency, gold, and foreign currency are insured. The coverage limits have changed 
several times over time: Between October 1986 and March 1992 the coverage was 100\% of TL 3 millions and $60 \%$ of the next TL 3 millions; between March 1992 and April 1994 the coverage was 100\% of TL 25 millions and $60 \%$ of the next TL 25 millions; between April 1994 and May 1994 the coverage was TL 150 millions without co-insurance. In the wake of the crises in 1994, all deposits have been brought under coverage between May 1994 and June 2000. Between June 2000 and December 2000 the coverage was TL 100 billions which then reduced to TL 50 billions in January 2001 just to be replaced by another blanket guarantee between July 2003 and July 2004. Since then coverage limit remained at TL 50 billions.

Sources: Central Bank of Turkey (1983), Own survey of deposit insurers, IADI Survey: Turkey (2003).

Turkmenistan. In 2000 Turkmenistan introduced a full guarantee on deposits including those denominated in foreign-currency. It is officially administered and has a compulsory membership policy.

Source: Barth, Caprio, and Levine (2004).

Uganda. (Deposit Insurance Fund, Financial Institutions Act, 1993) The fund in Uganda was established in 1994. It is officially administered by the Bank of Uganda and jointly funded. Membership is mandatory for all banks and credit institutions and they are required to pay a $0.2 \%$ flat rate assessed annually on weighted deposit liabilities. The coverage is U Sh 3 millions per depositor per institution. Foreign currency and inter-bank deposits are not covered.

Sources: Bank of Uganda (2004), IADI Survey: Uganda (2002).

Ukraine. (Fund for the Guarantee of Deposits of Natural Persons, Decree 996/98) The deposit guarantee scheme of Ukraine was established in September 1998. It is officially administered and jointly funded. The initial capital of UAH 20 million was provided by the National Bank of Ukraine and will lend when necessary. Deposits of insiders and their families, as well as inter-bank deposits are excluded. The coverage limit was initially set at UAH 1,200, which was raised to UAH 1,500 in 2003.

Source: Garcia (1999), IADI Member Profiles: Deposit Guarantee Fund-Ukraine.

United Kingdom. (Deposit Protection Fund, Banking Act of 1979 and 1987; Financial Services Compensation Scheme) The fund in the UK was established in 1982. The system is government legislated and privately administered and funded. The central bank made loans in the past but there is now no public funding for the DIS. There is no permanent fund in place and membership is mandatory. Banks make expost contributions when needed. Deposits of financial institutions are not covered by the system. The coverage limits have evolved as follows over time: in 1982 compensation limit was $75 \%$ of first $\$ 10,000$ which was raised to $75 \%$ of $£ 20,000$ in May 1987. In July 1995, the Scheme was amended by the Credit Institutions Regulations and maximum payment was changed to $90 \%$ of $£ 20,000$ or EUR 20,000 , whichever is higher. The Financial Services Compensation Scheme came into existence in December 2001 and the scheme changed the coverage to $100 \%$ of the first $£ 2,000$ and $90 \%$ of the next $£ 33,000$. Currently, deposits in all currencies are covered on a per depositor per institution basis.

Sources: Own survey of deposit insurers, Garcia (1999), IADI Survey: UK (2003), Kyei (1995).

United States. (Federal Deposit Insurance Corporation-FDIC, Federal Reserve Act) The US deposit insurance system was established in 1934 in response to the Great Depression. It is government legislated and administered and jointly funded. The government provided initial capital, borne losses of the savings \& loan associations in the past. Membership is compulsory for nationally chartered and for almost all statechartered banks and thrifts. Premiums are risk-adjusted and can range all the way from $0 \%$ to $0.27 \%$. Deposits booked off-shore are not covered. Initially the coverage limit was set at $\$ 5,000$. The coverage limit has been increased several times as follows: $\$ 10,000$ in $1950, \$ 15,000$ in $1966, \$ 20,000$ in 1969 , $\$ 40,000$ in 1974, and finally $\$ 100,000$ in 1980 .

Sources: Federal Deposit Insurance Corporation (1996), Garcia (1999), IADI Survey: USA (2003), and Kyei (1995).

Uruguay. (Bank Deposits Collateral Fund, Superintendency of Bank Savings Protection) Law on protection of bank deposits was enacted on December 27 $7^{\text {th }}, 2002$ creating a Bank Deposits Collateral Fund and a Superintendency of Bank Savings Protection. However, although Uruguay has established a deposit insurance system, it is not yet regulated. The Financial System Restructuring Act in Uruguay authorizes the executive branch to set aside part of its resources to cover deposits up to US\$100,000.

Sources: Central Bank of Uruguay (2002), IADB (2005). 
Venezuela. (Guarantee Fund of Deposits and Banking Protection-FOGADE, BANAP, Charter of Deposit Guarantee and Bank Protection Fund) The fund in Venezuela was established in 1985. It is officially administered and jointly funded. Central bank and government have borne losses and have refinanced the DIS in the past. The board has seven members of which four are from the government, one from the banks, one from the labor union, and one from the insurance agency's employees. In 1994 the premiums were raised from $0.5 \%$ to $2.0 \%$ due to a substantial assistance to troubled banks. The Fund has selectively made payments over the legally stated limits. Inter-bank and foreign currency deposits are not covered. The coverage limit since 2002 is Bs 10 millions, which was Bs 250,000 in 1985, Bs 1 mil in 1994, and Bs 4 mil in 1995.

Sources: FOGADE (2004), Garcia (1999), Kyei (1995).

Vietnam. (Deposit Insurance of Vietnam) Deposit Insurance of Vietnam was created in July 2000. It is government legislated and administered. The maximum coverage has been VND 30 millions which is calculated per depositor per institution.

Source: Own survey of deposit insurers.

Zimbabwe. The deposit insurance system of Zimbabwe was created in July 2003. The coverage limit was Zimbabwe $\$ 200,000$ as of 2003 . There is no co-insurance and coverage is calculated per depositor per institution. It is jointly administered.

Source: Barth, Caprio, and Levine (2004), Own survey of deposit insurers. 


\section{A.3 Data sources}

Agency for Restructuring Credit Organizations, Russia. 2004. Deposit Insurance Program. http://www.gkarco.ru/eng/pr gar.html

Allrefer.com Country Study and Guide: Sri Lanka. http://reference.allrefer.com/country-guide-study/srilanka

Alawode, Abayomi A. 1992. "Deposit Insurance in Nigeria: A Critical Appraisal," Nigerian Financial Review, v.5 no.4.

Asiatic Society of Bangladesh. 2004. Bangladesh Bank. http://banglapedia.search.com.bd/HT/B 0153.HTM

Bahrain Monetary Agency. 2004. Deposit Insurance Scheme. http://www.bma.gov.bh/cms/index.jsp?action=article \&ID $=37$

Bank of Albania. 2002. "On the Creation of the Insurance Deposits Agency," October $28^{\text {th }}$, press release.

Bank of Latvia. 1998. "Law on Natural Person Deposit Guarantees,” unofficial translation.

Bank of Uganda. 2004. In the Matter of the Financial Institutions Statute 1993.

Bank Rehabilitation Agency, Serbia. 2004. http://www.bra.gov.yu/english/

Banque du Liban. 2004. National Deposit Guarantee Institution. http://www.bdl.gov.lb/bfs/bfsparticipants/SB/ndgi.htm

Barth, James R., Gerard Caprio, and Ross Levine. 2004. "The Regulation and Supervision of Banks around the World: A New Database," 2003 version, Update. Washington, DC: World Bank.

Brance, Marta de Castello, Alfred Kammer, and Efiie Psalida. 1996. "Financial Sector Reform and Banking and Banking Crises in the Baltic Countries", IMF Working Paper, December, Washington, DC: International Monetary Fund.

Bruyneel, Andre, and Axel Miller. 1995. "Belgium Implements Deposit Guarantee-Scheme,” International Financial Law Review, London.

Central Bank of Kenya. 2004. Deposit Protection Fund Board. http://www.centralbank.go.ke/dpf/dpfindex.html

Central Bank of Turkey. 1983. "Law on Deposit Insurance Fund," unofficial translation.

Central Bank of Uruguay. 2002. Law No. 17.613, December 27.

Central European. 1998. "Bulgaria Introduces Law on Bank Deposits," May, London.

Deposit Insurance and Credit Guarantee Corporation, India. 2004. http://www.dicgc.org.in/

Deposit Insurance Fund Skopje, Republic of Macedonia. 2004. http://www.fodsk.org.mk/eng/default.asp

Federal Deposit Insurance Corporation, USA. 1996. Annual Report.

Fonds de Garantie des Depots, France. 1999. Regulation 99-05. http://www.garantiedesdepots.fr/spip/reglements 99 05.php

Garcia, Gillian. 1999. "Deposit Insurance: Actual and Best Practices," IMF Working Paper 99/54, Washington, DC: International Monetary Fund.

Garcia, Gillian. 2000. "Deposit Insurance: Actual and Good Practices," Occasional Paper No. 197, Washington, DC: International Monetary Fund.

Garcia, Gillian, and Henriette Prast. 2003. "Depositor and Investor Protection in the EU and the Netherlands: A Brief History," Journal of European Economic History, 2003(2).

Gibraltar Deposit Guarantee Board. 1998. Deposit Guarantee Scheme Ordinance. http://www.gdgb.gi/scheme.htm

Gerdrup, Karsten R. 2003. "Three Episodes of Financial Fragility in Norway since the 1890s," BIS Working Paper No. 142, Basel: Bank for International Settlements.

Guarantee Fund of Deposits and Banking Protection, Venezueala. 2004. http://www.fogade.gov.ve

Hellenistic Deposit Guarantee Fund, Greece. 2000. Law 2832/2000. http://www.hdgf.gr/binary/hdgf Law.pdf

Institute of International Bankers. Global Surveys 1995, 1996, 1997, 1998, 1999.

Inter-American Development Bank. 2005. "Designing Deposit Insurance," in IPES 2005: Unlocking Credit: The Quest for Deep and STable Bank Lending, Chapter 7, Washington, DC: Inter-American Development Bank.

International Association of Deposit Insurers (IADI) Member Profiles.

Albanian Deposit Insurance Agency

http://www.iadi.org/html/App/SiteContent/Member\%20Profile\%20Albania.pdf

Deposit Insurance Fund, Kazakhstan

http://www.iadi.org/html/App/SiteContent/Member\%20profile\%20Kazakhstan.pdf 
International Association of Deposit Insurers (IADI) Member Profiles.

Deposit Guarantee Fund, Ukraine

http://www.iadi.org/html/App/SiteContent/Member\%20Profile\%20Ukraine.pdf

International Association of Deposit Insurers (IADI) Survey.

Deposit Insurance Corporation, Bahamas, 2003

http://www.iadi.org/html/App/SiteContent/Complete\%20Surveys/Feb\%202003\%20-

\%20IDIS\%20DB/DIC\%20-\%20Bahamas\%20FullQ1A1.PDF

Deposit Insurance Agency, Bosnia and Herzegovina, 2002

http://www.iadi.org/html/App/SiteContent/Complete\%20Surveys/Feb\%202003\%20\%20IDIS\%20DB/DIAFBH\%20-\%20Bosnia\%20Herzogovinia\%20FullQ1A1.PDF

Central Bank, Chile, 2003

http://www.iadi.org/html/App/SiteContent/Complete\%20Surveys/Feb\%202003\%20-

\%20IDIS\%20DB/BCC\%20-\%20Chile\%20FullQ1A1.PDF

Central Bank, Cyprus, 2003

http://www.iadi.org/html/App/SiteContent/Incomplete\%20Surveys/CBC\%20-

\%20Cyprus\%20FullQ1A1.PDF

Deposit Insurance Fund, Czech Republic, 2003

http://www.iadi.org/html/App/SiteContent/Complete\%20Surveys/Feb\%202003\%20-

\%20IDIS\%20DB/CDIF\%20-\%20Czech\%20Republic\%20Full\%20Q1A1.PDF

Deposit Guarantee Fund, Denmark, 2002

http://www.iadi.org/html/App/SiteContent/Complete\%20Surveys/Feb\%202003\%20-

\%20IDIS\%20DB/DGFDI\%20-\%20Denmark\%20FullQ1A1.PDF

Deposit Guarantee Agency, Ecuador, 2003

http://www.iadi.org/html/App/SiteContent/Incomplete\%20Surveys/AGD\%20-

$\% 20$ Ecuador\%20FullQ1A1.PDF

Deposit Guarantee Institution, El Salvador, 2003

http://www.iadi.org/html/App/SiteContent/Incomplete\%20Surveys/IGD\%20-

$\% 20 \mathrm{El} \% 20$ Salvador\%20FullQ1A1.PDF

Deposit Guarantee Fund, Finland, 2002

http://www.iadi.org/html/App/SiteContent/Complete\%20Surveys/Feb\%202003\%20\%20IDIS\%20DB/DGF\%20-\%20Finland\%20FullQ1A1.PDF

Deposit Guarantee Fund, Greece, 2002

http://www.iadi.org/html/App/SiteContent/Complete\%20Surveys/Feb\%202003\%20$\% 20 I D I S \% 20 D B / F G C \% 20-\% 20$ Greece $\% 20$ FullQ1A1.PDF

Monetary Authority, Hong Kong, 2003

http://www.iadi.org/html/App/SiteContent/Incomplete\%20Surveys/HKMA\%20-

\%20Hong\%20Kong\%20FullQ1A1.PDF

National Deposit Insurance Fund, Hungary, 2003

http://www.iadi.org/html/App/SiteContent/Incomplete\%20Surveys/NDIFH\%20-

\%20Hungary\%20FullQ1A1.PDF

Depositors' and Investors' Guarantee Fund, Iceland, 2003

http://www.iadi.org/html/App/SiteContent/Incomplete\%20Surveys/DIGF\%20-

\%20Iceland\%20FullQ1A1.PDF

Financial Supervision Commission, Isle of Man, 2002

http://www.iadi.org/html/App/SiteContent/Complete\%20Surveys/Feb\%202003\%20-

\%20IDIS\%20DB/FSC\%20-\%20Isle\%20of\%20Man\%20FullQ1A1.PDF

Fondo Interbancario di Tutela dei Depositi, Italy, 2003

http://www.iadi.org/html/App/SiteContent/Incomplete\%20Surveys/FITD\%20-

\%20Italy\%20FullQ1A1.PDF

Deposit Insurance Corporation, Jamaica, 2003

http://www.iadi.org/html/App/SiteContent/Complete\%20Surveys/Feb\%202003\%20-

\%20IDIS\%20DB/JDIC\%20-\%20Jamaica\%20Full\%20Q1A3.pdf

Deposit Insurance Corporation, Japan, 2003

http://www.iadi.org/html/App/SiteContent/Incomplete\%20Surveys/DICJ\%20-

\%20Japan\%20FullQ1A1.PDF 
International Association of Deposit Insurers (IADI) Survey.

Deposit Insurance Corporation, Jordan, 2002

http://www.iadi.org/html/App/SiteContent/Complete\%20Surveys/Feb\%202003\%20\%20IDIS\%20DB/DIC\%20-\%20Jordan\%20FullQ1A1.PDF

Deposit Insurance Corporation, Korea, 2003

http://www.iadi.org/html/App/SiteContent/Incomplete\%20Surveys/KDIC\%20-

$\% 20$ Korea\%20FullQ1A1.PDF

Deposit Insurance Fund, Lithuania, 2002

http://www.iadi.org/html/App/SiteContent/Complete\%20Surveys/Feb\%202003\%20\%20IDIS\%20DB/DIFL\%20-\%20Lithuania\%20FullQ1A1.PDF

Deposit Insurance Fund, Macedonia, 2002

http://www.iadi.org/html/App/SiteContent/Complete\%20Surveys/Feb\%202003\%20\%20IDIS\%20DB/DIF\%20-\%20Macedonia\%20FullQ1A1.PDF

Bank Savings Protection Institute, Mexico, 2003.

http://www.iadi.org/html/App/SiteContent/Complete\%20Surveys/Feb\%202003\%20\%20IDIS\%20DB/IPAB\%20-\%20Mexico\%20FullQ1A1.PDF

Deposit Insurance Corporation, Nigeria, 2003

http://www.iadi.org/html/App/SiteContent/Complete\%20Surveys/Feb\%202003\%20\%20IDIS\%20DB/NDIC\%20-\%20Nigeria\%20Full\%20Q1A1.PDF

Savings Banks Guarantee Fund, Norway, 2003

http://www.iadi.org/html/App/SiteContent/Incomplete\%20Surveys/NSBGF\%20-

$\% 20$ Norway\%20FullQ1A1.PDF

Deposit Insurance Fund, Peru, 2003

http://www.iadi.org/html/App/SiteContent/Complete\%20Surveys/Feb\%202003\%20\%20IDIS\%20DB/FSD\%20-\%20Peru\%20FullQ1A1.PDF

Deposit Insurance Corporation, Philippines, 2003

http://www.iadi.org/html/App/SiteContent/Complete\%20Surveys/Feb\%202003\%20\%20IDIS\%20DB/PDIC\%20-\%20Philippines\%20FullQ1A1.PDF

Deposit Guarantee Fund, Portugal, 2002

http://www.iadi.org/html/App/SiteContent/Complete\%20Surveys/Feb\%202003\%20\%20IDIS\%20DB/FGD\%20-\%20Portugal\%20FullQ1A1.PDF

Deposit Guarantee Fund, Romania, 2002

http://www.iadi.org/html/App/SiteContent/Complete\%20Surveys/Feb\%202003\%20\%20IDIS\%20DB/DGFBS\%20-\%20Romania\%20FullQ1A1.PDF

Bank of Slovenia, 2003

http://www.iadi.org/html/App/SiteContent/Complete\%20Surveys/Feb\%202003\%20\%20IDIS\%20DB/BS\%20-\%20Slovenia\%20Full\%20Q1A2.PDF

Deposit Guarantee Fund, Spain, 2003

http://www.iadi.org/html/App/SiteContent/Complete\%20Surveys/Feb\%202003\%20\%20IDIS\%20DB/FGDCA\%20-\%20Spain\%20FullQ1A1.PDF

Deposit Guarantee Board, Sweden, 2003

http://www.iadi.org/html/App/SiteContent/Incomplete\%20Surveys/SDGB\%20-

$\% 20$ Sweden\%20FullQ1A1.PDF

Central Deposit Insurance Corporation, Taiwan, 2003

http://www.iadi.org/html/App/SiteContent/Complete\%20Surveys/Feb\%202003\%20\%20IDIS\%20DB/CDIC\%20-\%20Taiwan\%20Full\%20Q1A3.pdf

Deposit Insurance Board, Tanzania, 2003

http://www.iadi.org/html/App/SiteContent/Incomplete $\% 20$ Surveys/DIB-BT\%20\%20Tanzania\%20FullQ1A1.PDF

Deposit Insurance Corporation, Trinidad and Tobago, 2003

http://www.iadi.org/html/App/SiteContent/Incomplete\%20Surveys/DIC\%20-

\%20Trinidad\%20\&\%20Tobago\%20FullQ1A1.PDF

Deposit Insurance Fund, Turkey, 2003

http://www.iadi.org/html/App/SiteContent/Complete\%20Surveys/Feb\%202003\%20\%20IDIS\%20DB/BRSA\%20-\%20Turkey\%20FullQ1A1.PDF 
International Association of Deposit Insurers (IADI) Survey.

Deposit Insurance Fund, Uganda, 2002

http://www.iadi.org/html/App/SiteContent/Complete\%20Surveys/Feb\%202003\%20-

\%20IDIS\%20DB/BU\%20-\%20Uganda\%20FullQ1A1.PDF

Financial Services Compensation Scheme, United Kingdom, 2003

http://www.iadi.org/html/App/SiteContent/Incomplete\%20Surveys/FSCS\%20-

$\% 20 \mathrm{UK} \% 20 \mathrm{FullQ1A} 1 . \mathrm{PDF}$

Federal Deposit Insurance Corporation, USA, 2003

http://www.iadi.org/html/App/SiteContent/Complete\%20Surveys/Feb\%202003\%20-

\%20IDIS\%20DB/FDIC\%20-\%20USA\%20FullQ1A1.PDF

International Monetary Fund Country Report, Lithuania. 2002. "Republic of Lithuania: Financial System

Stability Assessment, including Reports on the Observance of Standards and Codes on the following topics:

Monetary and Financial Policy Transparency, Banking Supervision, Insurance Regulation, and Payment

Systems," IMF Country Report No. 02/19, February, Washington, DC: International Monetary Fund.

International Monetary Fund Country Report, Macedonia. 2003. "Former Yugoslav Republic of Macedonia: Financial System Stability Assessment, including Reports on the Observance of Standards and Codes on the following topics: Banking Supervision, Payment Systems, Monetary and Financial Policy Transparency, and Anti-Money Laundering and Combating the Financing of Terrorism,” IMF Country Report No. 03/374, November, Washington, DC: International Monetary Fund.

International Monetary Fund Factsheet. 1998. "IMF Bail Outs: Truth and Fiction,” January, Washington, DC: International Monetary Fund.

Ioannidou, Vasso P., and Jan de Dreu. 2004. "The Impact of Explicit Deposit Insurance on Market Discipline," mimeo, Tilburg University.

Kazakhstan Deposit Insurance Fund. 2004. http://www.kdif.kz/index.php?page $=1 \& g o=$ info\&lang_chose $=$ en

Ko, Don Wong. 1997. "Korea Introduces Banks Deposit Insurance Scheme," International Financial Law Review, London, April.

Korea Deposit Insurance Corporation. 2004. http://www.kdic.or.kr/english/

Kyei, Alexander. 1995. "Deposit Protection Arrangements: A Comparative Study," IMF Working Paper 95/134, Washington, DC: International Monetary Fund.

Law on Bank Deposit Guaranty, Bulgaria. 1998. State Gazette issue 49, 29 April 1998; final amendment issue 118 of 2002.

Liechtenstein Banking Act. 1992. Unofficial translation.

Malta Financial Services Authority. 2004. The Depositor Guarantee and the Investor Compensation Scheme. http://www.mfsa.com.mt/mfsa/default.asp

Miller, Geoffrey P. 1993. "Politics of Deposit Insurance Reform: The Case of Argentina," FDICIA: An Appraisal, Proceedings of the $29^{\text {th }}$ Conference on Bank Structure and Competition, Federal Reserve Bank of Chicago.

Ministry of Finance Hungary. 1993. Act on National Deposit Insurance Fund.

Organization for Economic Co-operation and Development Economic Surveys: Russian Federation. 2004. "OECD Economic Survey of the Russian Federation 2004: Russia's Deposit Insurance Law," OECD.

Oxford Analytica Brief. (1995). “Argentina: Banking Shakeout,” April 20.

Oxford Analytica Brief. (1997). "Japan: Stimulation Package," December.

Research Center of the Institute for Privatization and Management. 2003. "Proposals for Further Development of the Deposit Insurance System in Belarus," PP/2/03, German Economic Team in Belarus.

SEDESA (Seguro de Depositos S.A.) Argentina. 2003. http://200.61.172.161./ english/index.php?dir=sobre\&file=cober

Talley, Samuel H., and Ignacio Mas. 1990. "Deposit Insurance in Developing Countries," Policy Research Working Paper, No. 548, Washington, DC: World Bank.

Talley, Samuel H. 1998. "An Advisory Report on Brazil's Banking Sector Safety Net and The Role of Deposit Insurance," World Bank Advisory Report, Washington, DC: World Bank.

U.S. Embassy Reports. 1998. "Estonia - April 1998 Economic Highlights", CEEBICnet, April.

Valori, Veli-Pekka, and Jukka Vesala. 1998. "Reform of the Finnish Deposit Guarantee Scheme" Bank of Finland Bulletin, Vol. 72, No.3, March. 